\title{
QUASICONFORMAL MAPS, ANALYTIC CAPACITY, AND NON LINEAR POTENTIALS
}

\author{
XAVIER TOLSA AND IGNACIO URIARTE-TUERO
}

\begin{abstract}
In this paper we prove that if $\phi: \mathbb{C} \rightarrow \mathbb{C}$ is a $K$-quasiconformal map, with $K>1$, and $E \subset \mathbb{C}$ is a compact set contained in a ball $B$, then

$$
\frac{\dot{C}_{\frac{2 K}{2 K+1}} \frac{2 K+1}{K+1}(E)}{\operatorname{diam}(B)^{\frac{2}{K+1}}} \geq c^{-1}\left(\frac{\gamma(\phi(E))}{\operatorname{diam}(\phi(B))}\right)^{\frac{2 K}{K+1}},
$$

where $\gamma$ stands for the analytic capacity and $\dot{C}_{\frac{2 K}{2 K+1}}, \frac{2 K+1}{K+1}$ is a capacity associated to a non linear Riesz potential. As a consequence, if $E$ is not $K$-removable (i.e. removable for bounded $K$-quasiregular maps), it has positive capacity $\dot{C}_{\frac{2 K}{2 K+1}} \frac{2 K+1}{K+1}$. This improves previous results that assert that $E$ must have non $\sigma$-finite Hausdorff measure of dimension $2 /(K+1)$. We also show that the indices $\frac{2 K}{2 K+1}, \frac{2 K+1}{K+1}$ are sharp, and that Hausdorff gauge functions do not appropriately discriminate which sets are $K$-removable. So essentially we solve the problem of finding sharp "metric" conditions for $K$-removability.
\end{abstract}

\section{INTRODUCTION}

A homeomorphism $\phi: \Omega \rightarrow \Omega^{\prime}$ between planar domains is called $K$-quasiconformal if it preserves orientation, it belongs to the Sobolev space $W_{\text {loc }}^{1,2}(\Omega)$, and satisfies

$$
\max _{\alpha}\left|\partial_{\alpha} \phi\right| \leq K \min _{\alpha}\left|\partial_{\alpha} \phi\right| \quad \text { a.e. in } \Omega \text {. }
$$

If one does not ask $\phi$ to be a homeomorphism, then one says that $\phi$ is quasiregular. When $K=1$, the class of quasiregular maps coincides with the one of analytic functions.

A compact set $E \subset \mathbb{C}$ is said to be removable for bounded $K$-quasiregular maps (or, $K$-removable) if for every open set $\Omega \supset E$, every bounded $K$-quasiregular map $f: \Omega \backslash E \rightarrow \mathbb{C}$ admits a $K$-quasiregular extension to $\Omega$. By Stoilow's theorem, it turns out that $E$ is $K$-removable if, and only if, for every planar $K$ quasiconformal map $\phi, \phi(E)$ is removable for bounded analytic functions (i.e. $\phi(E)$

2000 Mathematical Subject Classification. .

Key words and phrases. Quasiconformal mappings in the plane, analytic capacity.

X. T. is partially supported by grants MTM2007-62817, MTM2010-16232 (Spain), and 2009SGR-420 (Catalonia). I. U. was a postdoctoral fellow at the University of Missouri, Columbia, USA, and at Centre de Recerca Matemàtica, Barcelona, Spain, for some periods of time during the elaboration of this paper. During the elaboration of this paper, he has been partially supported by grants DMS-0901524, CAREER DMS-1056965 (US NSF), Sloan Research Foundation, and MTM2010-16232, MTM2009-14694-C02-01 (Spain). 
is 1-removable). The Painlevé problem for $K$-quasiregular mappings consists in describing $K$-removable sets in metric and geometric terms, in analogy to the classical Painlevé problem of characterizing removable sets for bounded analytic functions in metric and geometric terms (i.e. precisely the case $K=1)$. In this case $(K=1)$, a "solution" in terms of the so called curvature of measures was obtained in [Tol03] (see also [Tol05]).

The analytic capacity of a compact set $E \subset \mathbb{C}$ is defined by

$$
\gamma(E)=\sup _{f}\left|f^{\prime}(\infty)\right|
$$

where the supremum is taken over all bounded analytic functions $f: \mathbb{C} \backslash E \rightarrow \mathbb{C}$ with $\|f\|_{\infty} \leq 1$, and

$$
f^{\prime}(\infty)=\lim _{z \rightarrow \infty} z(f(z)-f(\infty))
$$

This set function was introduced by Ahlfors in order to study the Painlevé problem for bounded analytic functions. He showed that $E$ is removable for these functions if and only if $\gamma(E)=0$. By the relationship between 1-removable and $K$-removable sets explained above, it turns out that $E$ is $K$-removable if and only if $\gamma(\phi(E))=0$ for all planar $K$-quasiconformal maps.

An old theorem of Painlevé shows that $\gamma(E) \lesssim \mathcal{H}^{1}(E)$ for any compact set $E$, where $\mathcal{H}^{1}(E)$ denotes the one-dimensional Hausdorff measure (length) of $E$. This result gives the sharp condition in terms of Hausdorff measures for removability for bounded analytic functions, since sets of zero length are removable, but there are sets of positive length which are not removable (e.g. a line segment.)

By Painlevé's theorem, if $\gamma(\phi(E))>0$, then $\phi(E)$ has positive length, and so it has Hausdorff dimension at least 1. By the celebrated theorem of Astala on the distortion of area Ast94, this forces the Hausdorff dimension of $E$ to be at least $2 /(K+1)$. Quite recently, in $\left[\mathrm{ACM}^{+} 08\right]$ it was shown that, in fact, the $\frac{2}{K+1}$-Hausdorff measure $\mathcal{H}^{\frac{2}{K+1}}(E)$ must be positive and, moreover, non $\sigma$-finite, or, equivalently, if $\mathcal{H}^{\frac{2}{K+1}}(E)$ is $\sigma$-finite, then $E$ is $K$-removable. To get this result, the authors proved, on the one hand, that if $\mathcal{H}^{1}(\phi(E))$ is non $\sigma$-finite, then $\mathcal{H}^{\frac{2}{K+1}}(E)$ is also non $\sigma$-finite, equivalently, if $\mathcal{H}^{\frac{2}{K+1}}(E)$ is $\sigma$-finite, then $\mathcal{H}^{1}(\phi(E))$ is $\sigma$-finite (see [LSUT10] for related recent results). On the other hand, if $\mathcal{H}^{1}(\phi(E))$ is $\sigma$-finite and $\gamma(\phi(E))>0$, from David's solution of Vitushkin's conjecture [Dav98] and the countable semiadditivity of analytic capacity [Tol03], it turns out that $\phi(E)$ contains some rectifiable subset of positive length. Using improved distortion estimates for the dimension of rectifiable sets, the authors showed that in this case the Hausdorff dimension of $E$ must be strictly larger that $2 /(K+1)$, and so $\mathcal{H}^{\frac{2}{K+1}}(E)$ is also non $\sigma$-finite in this case. 
Moreover, in $\left[\mathrm{ACM}^{+} 08\right]$ it was also shown (see Theorem 8.2 below) that for any measure function $h(t)=t^{\frac{2}{K+1}} \varepsilon(t)$ such that

$$
\int_{0} \frac{\varepsilon(t)^{1+1 / K}}{t} d t<\infty
$$

there is a compact set $E$ which is not $K$-removable and such that $0<\mathcal{H}^{h}(E)<\infty$. In particular, whenever $\varepsilon(t)$ is chosen so that in addition for every $\alpha>0$ we have $t^{\alpha} / \varepsilon(t) \rightarrow 0$ as $t \rightarrow 0$, then there is a non- $K$-removable set $E$ with $\operatorname{dim}(E)=$ $\frac{2}{K+1}$. Note that there is a small "gap" between the sufficient condition for the gauge function $h$ for the existence of non- $K$-removable sets just mentioned, and the sufficient condition for $K$-removability of sets also from [ACM ${ }^{+} 08$ ] mentioned before (namely that $\mathcal{H}^{\frac{2}{K+1}}(E)$ be $\sigma$-finite, see Theorem 8.1 below.)

The main result of this paper improves on the preceding results, giving the sharp "metric" condition for $K$-removability in terms of Riesz capacities (i.e. closing the aforementioned "gap"):

Theorem 1.1. Let $E \subset \mathbb{C}$ be compact and $\phi: \mathbb{C} \rightarrow \mathbb{C}$ a $K$-quasiconformal mapping, $K>1$. If $E$ is contained in a ball $B$, then

$$
\frac{\dot{C}_{\frac{2 K}{2 K+1}, \frac{2 K+1}{K+1}}(E)}{\operatorname{diam}(B)^{\frac{2}{K+1}}} \geq c^{-1}\left(\frac{\gamma(\phi(E))}{\operatorname{diam}(\phi(B))}\right)^{\frac{2 K}{K+1}} .
$$

In this theorem, the constant $c$ depends only on $K$. On the other hand, $\dot{C}_{\frac{2 K}{2 K+1}}, \frac{2 K+1}{K+1}$ is a Riesz capacity associated to a non linear potential. Recall that, for $\alpha>0$, $1<p<\infty$ with $0<\alpha p<2$, the Riesz capacity $\dot{C}_{\alpha, p}$ of $F$ is defined as

$$
\dot{C}_{\alpha, p}(F)=\sup _{\mu} \mu(F)^{p}
$$

where the supremum runs over all positive measures $\mu$ supported on $F$ such that

$$
I_{\alpha}(\mu)(x)=\int \frac{1}{|x-y|^{2-\alpha}} d \mu(x)
$$

satisfies $\left\|I_{\alpha}(\mu)\right\|_{p^{\prime}} \leq 1$, where as usual $p^{\prime}=p /(p-1)$.

It is easy to check that $\dot{C}_{\alpha, p}$ is a homogeneous capacity of degree $2-\alpha p$, that is,

$$
\dot{C}_{\alpha, p}(\lambda F)=|\lambda|^{2-\alpha p} \dot{C}_{\alpha, p}(F)
$$

for any compact set $F \subset \mathbb{C}$ and $\lambda \in \mathbb{C}$. Therefore, $\dot{C}_{\frac{2 K}{2 K+1}}, \frac{2 K+1}{K+1}$ has homogeneity $2 /(K+1)$. The indices $\alpha=\frac{2 K}{2 K+1}, p=\frac{2 K+1}{K+1}$, are sharp and cannot be improved in the theorem. See Theorem 8.8 below for a more precise statement.

It is well known that sets with positive capacity $\dot{C}_{\alpha, p}$ have non $\sigma$-finite Hausdorff measure $\mathcal{H}^{2-\alpha p}$. So as a direct corollary of Theorem 1.1 one recovers the result of $\left[\mathrm{ACM}^{+} 08\right.$ ] that asserts that if $\gamma(\phi(E))>0$, then $\mathcal{H}^{\frac{2}{K+1}}(E)$ is non $\sigma$-finite, or, as phrased in $\left[\mathrm{ACM}^{+} 08\right]$, if $\mathcal{H}^{\frac{2}{K+1}}(E)$ is $\sigma$-finite, then $\gamma(\phi(E))=0$. 
On the other hand, not all sets with non $\sigma$-finite $\mathcal{H}^{\frac{2}{K+1}}$ measure have positive capacity $\dot{C}_{\frac{2 K}{2 K+1}}, \frac{2 K+1}{K+1}$. So Theorem 1.1 provides new examples of $K$-removable sets. More precisely, for any increasing nonnegative function $h$ on $[0, \infty)$, with

$$
\int_{0}^{1}\left(\frac{h(r)}{r^{\frac{2}{K+1}}}\right)^{1+\frac{1}{K}} \frac{d r}{r}=\infty,
$$

there is a compact set $E \subset \mathbb{C}$ such that the generalized Hausdorff measure $\mathcal{H}^{h}(E)>$ 0 and $\dot{C}_{\frac{2 K}{2 K+1}, \frac{2 K+1}{K+1}}(E)=0$. See Theorem 8.3 below.

However, for any positive function $h$ on $(0, \infty)$ such that

$$
\varepsilon(r)=\frac{h(r)}{r^{\frac{2}{K+1}}} \rightarrow 0 \quad \text { as } r \rightarrow 0,
$$

there is a compact set $E \subset \mathbb{C}$ such that $\mathcal{H}^{h}(E)=0$ and a $K$-quasiconformal map $\phi$ such that $\gamma(\phi E)>0$ (and hence $\dot{C}_{\frac{2 K}{2 K+1}}, \frac{2 K+1}{K+1}(E)>0$, due to Theorem 1.1.) See Section 8 (in particular, Theorem 8.5) for more details and examples.

The results just mentioned strongly suggest 1) that the "gap" left in $\left[\mathrm{ACM}^{+} 08\right.$ in terms of characterizing sharp "metric" conditions for $K$-removability cannot be closed in terms of Hausdorff gauge functions, 2) that the language of (Riesz) capacities is more appropriate to obtain sharp "metric" conditions for $K$-removability, and 3 ) that (in view of the aforementioned Theorem 8.8 below and the fact that we recover the previously known sufficient conditions for $K$-removability from $\left[\mathrm{ACM}^{+} 08\right]$ ), we close the "gap" by obtaining sharp "metric" conditions for $K$-removability in terms of Riesz capacities.

For our purposes, the description of Riesz capacities in terms of Wolff potentials is more useful than the above definition of $\dot{C}_{\alpha, p}$. Consider

$$
\dot{W}_{\alpha, p}^{\mu}(x)=\int_{0}^{\infty}\left(\frac{\mu(B(x, r))}{r^{2-\alpha p}}\right)^{p^{\prime}-1} \frac{d r}{r} .
$$

A well known theorem of Wolff asserts that

$$
\dot{C}_{\alpha, p}(F) \approx \sup _{\mu} \mu(F)
$$

where the supremum is taken over all measures $\mu$ supported on $F$ such that $\dot{W}_{\alpha, p}^{\mu}(x) \leq$ 1 for all $x \in F$. See [AH96, Chapter 4], for instance. Notice that for the indices $\alpha=\frac{2 K}{2 K+1}, p=\frac{2 K+1}{K+1}$, we have

$$
\dot{W}_{\alpha, p}^{\mu}(x)=\int_{0}^{\infty}\left(\frac{\mu(B(x, r))}{r^{\frac{2}{K+1}}}\right)^{\frac{K+1}{K}} \frac{d r}{r} .
$$

We will also prove the following result in this paper.

Theorem 1.2. Let $1<p<\infty, E \subset \mathbb{C}$ be compact and $\phi: \mathbb{C} \rightarrow \mathbb{C} a K$ quasiconformal mapping. Then, 
QUASICONFORMAL MAPS, ANALYTIC CAPACITY, AND NON LINEAR POTENTIALS 5

(a) If $E$ is contained in a ball $B$,

$$
\frac{\dot{C}_{\frac{2 K}{2 K p-K+1}}, \frac{2 K p-K+1}{K+1}(E)}{\operatorname{diam}(B)^{\frac{2}{K+1}}} \gtrsim\left(\frac{\dot{C}_{1 / p, p}(\phi(E))}{\operatorname{diam}(\phi(B))}\right)^{\frac{2 K}{K+1}} .
$$

(b) If $\phi$ is conformal outside $E, K$-quasiconformal in $\mathbb{C}$, and moreover, $\mid \phi(z)-$ $z \mid=O(1 /|z|)$ as $z \rightarrow \infty$, then

$$
\dot{C}_{1 / p, p}(E) \approx \dot{C}_{1 / p, p}(\phi(E))
$$

The constants in (1.1) and (1.2) only depend on $p, K$.

Notice that the capacity $\dot{C}_{1 / p, p}$ is homogeneous of degree 1 , while $\dot{C}_{\frac{2 K}{2 K p-K+1}}, \frac{2 K p-K+1}{K+1}$ is homogeneous of degree $2 /(K+1)$.

To understand the relationship between analytic capacity and non linear potentials, we need to recall the characterization of analytic capacity in terms of curvature. For $x \in \mathbb{C}$, denote

$$
c_{\mu}^{2}(x):=\iint \frac{1}{R(x, y, z)^{2}} d \mu(y) d \mu(z),
$$

where $R(x, y, z)$ stands for the radius of the circle through $x, y, z$ (with $R(x, y, z)=$ $\infty$ if the points are colinear).

Theorem A. For any compact $E \subset \mathbb{C}$ we have

$$
\gamma(E) \simeq \sup \mu(E)
$$

where the supremum is taken over all Borel measures $\mu$ supported on $E$ such that $\mu(B(x, r)) \leq r$ for all $x \in \mathbb{C}, r>0$ and $c_{\mu}^{2}(x) \leq 1$ for all $x \in \mathbb{C}$.

The inequality $\gamma(E) \gtrsim \sup \mu(E)$ is due to Melnikov [Mel95], while the (more difficult) converse was proved in Tol03].

It is easy to check that

$$
\left(\sup _{r>0} \frac{\mu(B(x, r))}{r}\right)^{2}+c_{\mu}^{2}(x) \leq C \sum_{k \in \mathbb{Z}}\left(\frac{\mu\left(B\left(x, 2^{k}\right)\right)}{2^{k}}\right)^{2} \leq C \dot{W}_{2 / 3,3 / 2}^{\mu}(x) .
$$

From this fact, one infers that

$$
\gamma(F) \geq c^{-1} \dot{C}_{2 / 3,3 / 2}(F)
$$

for every compact set $F$. On the other hand, Theorem 1.2 tells us that

$$
\dot{C}_{\frac{2 K}{2 K+1}, \frac{2 K+1}{K+1}}(E) \geq c^{-1}\left(\dot{C}_{2 / 3,3 / 2}(\phi(E))\right)^{\frac{2 K}{K+1}}
$$

(assuming $\operatorname{diam}(B)=\operatorname{diam}(\phi(B))=1)$. If the estimate $\gamma(F) \approx \dot{C}_{2 / 3,3 / 2}(F)$ were true, then Theorem 1.1 would follow from this and (1.5). However, the comparability of $\gamma$ and $\dot{C}_{2 / 3,3 / 2}$ is false (for instance, if $F$ is a segment, $\gamma(F)>0$, while $\dot{C}_{2 / 3,3 / 2}(F)=$ $0)$. 
Nevertheless, for Cantor type sets $F$ such as the ones considered in [Mat96] and MTV03] it is true that $\gamma(F) \approx \dot{C}_{2 / 3,3 / 2}(F)$. So for this type of sets, the estimate

$$
\frac{\dot{C}_{\frac{2 K}{2 K+1}}, \frac{2 K+1}{K+1}\left(\phi^{-1}(F)\right)}{\operatorname{diam}(B)^{\frac{2}{K+1}}} \geq c^{-1}\left(\frac{\gamma(F)}{\operatorname{diam}(\phi(B))}\right)^{\frac{2 K}{K+1}}
$$

is a direct consequence of Theorem 1.2. On the other hand, by the results in $\mathrm{ACM}^{+} 08$, if $F$ is rectifiable (and thus $\gamma(F)>0$ ), then the Hausdorff dimension of $\phi^{-1}(F)$ is strictly larger than $2 /(K+1)$, and so

$$
\dot{C}_{\frac{2 K}{2 K+1}, \frac{2 K+1}{K+1}}\left(\phi^{-1}(F)\right)>0
$$

The proof of Theorem 1.1 for general sets $E$ follows by combining the arguments in Theorem 1.2 with quantitative estimates for the distortion of rectifiable sets (more precisely, for the distortion of sub-arcs of chord arc curves). To this end, we will need to use a corona type construction similar to the one used in [Tol05] to prove the bilipschitz invariance of analytic capacity, modulo multiplicative estimates.

The relationship between capacities $\gamma_{\beta}$ associated to Calderón-Zygmund kernels of the form $x /|x|^{\beta+1}$ in $\mathbb{R}^{n}$ and the capacities $\dot{C}_{\alpha, p}$ was first observed by Mateu, Prat and Verdera [MPV05]. In this paper the authors proved that if $0<\beta<1$, then

$$
\gamma_{\beta} \approx \dot{C}_{(n-\beta) 2 / 3,3 / 2}
$$

An immediate consequence is that sets of positive but finite $\beta$-Hausdorff measure are removable for $\gamma_{\beta}$, as shown previously by Prat [Pra04]. In the case $n=2, \beta=1$, the capacity $\gamma_{\beta}$ coincides with the analytic capacity $\gamma$, modulo multiplicative constants, and the comparability (1.6) fails. Instead, only the inequality (1.4) holds. It is an open problem to prove (or disprove) that (1.6) holds for every non integer $\beta \in(0, n)$.

Other results concerning the relationship between the capacities $\dot{C}_{\alpha, p}$ and the so called Lipschitz harmonic capacity (which can be considered as a generalization of analytic capacity to higher dimensions) have been obtained recently in [ENV10]. See [Par90] and [Vol03] for more details on Lipschitz harmonic capacity.

The plan of the paper is the following: in next section we prove Theorem 1.2, while Sections 3-7 are devoted to the proof Theorem 1.1. Section 8 contains examples and results that illustrate the sharpness of our results, and in Section 9 there are some final remarks. As usual, the letters $c, C$ denote constants (often, absolute constants) that may change at different occurrences, while constants with subscript, such as $C_{1}$, retain their values. The notation $A \lesssim B$ means that there is a positive constant $C$ such that $A \leq C B$; and $A \approx B$ means that $A \lesssim B \lesssim A$. 


\section{Distortion estimates FOR NON Linear POTENTIALS}

2.1. Strategy for the proof of Theorem 1.2. Note that, that (1.1) holds for all $K$-quasiconformal maps is equivalent to

$$
\frac{\dot{C}_{\frac{2 K}{2 K p-K+1}}, \frac{2 K p-K+1}{K+1}(\phi(E))}{\operatorname{diam}(\phi(B))^{\frac{2}{K+1}}} \geq c^{-1}\left(\frac{\dot{C}_{1 / p, p}(E)}{\operatorname{diam}(B)}\right)^{\frac{2 K}{K+1}} \quad \text { if } E \subset B
$$

for all $K$-quasiconformal maps $\phi$. The difference between (1.1) and (2.1) is mostly "psychological": think of $\phi$ as taking a set $E$ of dimension 1 to a set of dimension

$\frac{2}{K+1}$, as opposed to the other way round (which would be looking at $\phi^{-1}$ ). The equivalence between (1.1) and (2.1) uses quasisymmetry.

Let $\mu$ be a measure supported on $E$ such that $\dot{W}_{1 / p, p}^{\mu}(x) \leq 1$ for all $x \in \mathbb{C}$. In a sense, we want to show how $\mu$ is distorted. A first attempt might consist in obtaining suitable estimates for the Wolff potentials associated to the image measure $\phi \mu$. However, we have not been able to follow this approach.

Instead, to prove (2.1), we have transformed our original problem of estimating distortion in terms of Riesz capacities into another involving "Hausdorff-like" measures or contents. As far as we know, this way of studying the Riesz capacities is new. The advantage of this approach is that arguments using covering lemmas and related techniques are better suited for Hausdorff contents than for non linear potentials. In this way, some of the arguments in $\left[\mathrm{ACM}^{+} 08\right.$ will be adapted to estimate Riesz capacities via the intermediate contents of Hausdorff type that we will construct below.

Throughout all this section we suppose that $\mu$ is a finite Borel measure supported on $E$ such that $\dot{W}_{1 / p, p}^{\mu}(x) \leq 1$ for all $x \in \mathbb{C}$. In particular, notice that this implies that $\theta_{\mu}(B):=\mu(B) / r(B) \lesssim 1$ for any ball $B \subset \mathbb{C}$ with radius $r(B)$. We plan to introduce Hausdorff-like measures associated to $\mu$. To this end, first we need to define suitable gauge functions on all the balls in $\mathbb{C}$. Given a parameter $a>0$, we consider the function

$$
\psi_{a}(x)=\frac{1}{|x|^{1+a}+1}, \quad x \in \mathbb{C} .
$$

For the ball $B=B(x, t)$ we define

$$
\varepsilon_{\mu, a}(x, t)=\varepsilon_{\mu, a}(B):=\frac{1}{t} \int \psi_{a}\left(\frac{y-x}{t}\right) d \mu(y),
$$

and we consider the gauge function

$$
h_{\mu, a}(x, t)=h_{\mu, a}(B):=t \varepsilon_{\mu, a}(B) .
$$

Notice that $\varepsilon_{\mu, a}(B)$ and $h_{\mu, a}(B)$ can be considered as smooth versions of $\theta_{\mu}(B)$ and $\mu(B)$, respectively. One of the advantages of $\varepsilon_{\mu, a}(x, t)$ over $\theta_{\mu}(x, t)$ (where, of course, $\left.\theta_{\mu}(x, t):=\theta_{\mu}(B(x, t))\right)$ is that $\varepsilon_{\mu, a}(x, 2 t) \leq C \varepsilon_{\mu, a}(x, t)$ for any $x$ and $t>0$, which fails in general for $\theta_{\mu}(x, t)$. Analogously, we have $h_{\mu, a}(x, 2 t) \leq C h_{\mu, a}(x, t)$, while $\mu(B(x, t))$ and $\mu(B(x, 2 t))$ may be very different. 
Observe that, decomposing the integrals into annuli, for all $x \in \mathbb{C}$ we get

$$
\begin{aligned}
\int_{0}^{\infty} \varepsilon_{\mu, a}(x, t)^{p^{\prime}-1} \frac{d t}{t} & =\int_{0}^{\infty} \frac{1}{t^{p^{\prime}-1}}\left(\int \psi_{a}\left(\frac{y-x}{t}\right) d \mu(y)\right)^{p^{\prime}-1} \frac{d t}{t} \\
& \leq C \sum_{j \in \mathbb{Z}} 2^{-\left(p^{\prime}-1\right) j}\left(\sum_{k>j} \mu\left(B\left(x, 2^{k}\right)\right) 2^{(1+a)(j-k)}\right)^{p^{\prime}-1} \\
& \leq C \sum_{j \in \mathbb{Z}} 2^{-\left(p^{\prime}-1\right) j} \sum_{k>j} \mu\left(B\left(x, 2^{k}\right)\right)^{p^{\prime}-1} 2^{\left(p^{\prime}-1\right)\left(1+\frac{a}{2}\right)(j-k)}
\end{aligned}
$$

where we applied Hölder's inequality for $p^{\prime}-1>1$, and the fact that $(c+d)^{p^{\prime}-1} \leq$ $c^{p^{\prime}-1}+d^{p^{\prime}-1}$ otherwise. Thus,

$$
\int_{0}^{\infty} \varepsilon_{\mu, a}(x, t)^{p^{\prime}-1} \frac{d t}{t} \lesssim \sum_{k \in \mathbb{Z}} \mu\left(B\left(x, 2^{k}\right)\right)^{p^{\prime}-1} 2^{-\left(p^{\prime}-1\right)\left(1+\frac{a}{2}\right) k} \sum_{j<k} 2^{\left(p^{\prime}-1\right) \frac{a}{2} j} \lesssim \dot{W}_{1 / p, p}^{\mu}(x) \lesssim 1 .
$$

2.2. The contents $M^{h}$ and the families $\mathcal{G}_{1}$ and $\mathcal{G}_{2}$. Let $\mathcal{B}$ denote the family of all closed balls contained in $\mathbb{C}$. We consider a function $\varepsilon: \mathcal{B}: \rightarrow[0, \infty)$ (for instance, we can take $\left.\varepsilon=\varepsilon_{\mu, a}\right)$, and we define $h(x, r)=r \varepsilon(x, r)$. We assume that $\varepsilon, h$ are such that $h(x, r) \rightarrow 0$ as $r \rightarrow 0$, for all $x \in \mathbb{C}$. We introduce the measure $H^{h}$ following Carathéodory's construction (see Mat95], p.54): given $0<\delta \leq \infty$ and a set $F \subset \mathbb{C}$, we consider

$$
H_{\delta}^{h}(F)=\inf \sum_{i} h\left(B_{i}\right)
$$

where the infimum is taken over all coverings $F \subset \bigcup_{i} B_{i}$ with balls $B_{i}$ with radii smaller that $\delta$. Finally, we define

$$
H^{h}(F)=\lim _{\delta \rightarrow 0} H_{\delta}^{h}(F) .
$$

Recall that $H^{h}$ is a Borel regular measure (see [Mat95]), although it is not a "true" Hausdorff measure. For the $h$-content, we use the notation $M^{h}(E):=H_{\infty}^{h}(E)$.

We say that the function $\varepsilon$ belongs to $\mathcal{G}_{1}$ if it verifies the following properties for all balls $B(x, r), B(y, s)$ : there exists a constant $C_{0}$ such that if $|x-y| \leq 2 r$ and $r / 2 \leq s \leq 2 r$, then

$$
C_{0}^{-1} \varepsilon(x, r) \leq \varepsilon(y, s) \leq C_{0} \varepsilon(x, r) .
$$

If moreover, there exists $C_{0}^{\prime}$ such that

$$
\sum_{k \geq 0} 2^{-k} \varepsilon\left(x, 2^{k} r\right) \leq C_{0}^{\prime} \varepsilon(x, r)
$$

then we set $\varepsilon \in \mathcal{G}_{2}$.

Notice that (2.6) also holds with a different constant $C_{0}$ if one assumes $|x-y| \leq C r$ and $C^{-1} r \leq s \leq C r$. 
It is easy to check that the function $\varepsilon_{\mu, a}$ introduced above belongs to $\mathcal{G}_{1}$ for all $a>0$, and to $\mathcal{G}_{2}$ if $0<a<1$ (see Lemma 2.4 below for a stronger statement). Moreover, we have:

Lemma 2.1. If $\varepsilon \in \mathcal{G}_{1}$ and $h(x, r)=r \varepsilon(x, r)$, then Frostman's Lemma holds for $H^{h}$. That is to say, given a compact set $F \subset \mathbb{C}$, the following holds: $M^{h}(F)>0$ if and only if there exists a Borel measure $\nu$ supported on $F$ such that $\nu(B) \leq h(B)$ for any ball $B$. Moreover, one can find $\nu$ such that $\nu(F) \geq c^{-1} M^{h}(F)$.

The proof is almost the same as the one of the usual Frostman's Lemma (for instance, see [Mat95], p.112), taking into account the regularity properties of the gauge functions $h \in \mathcal{G}_{1}$.

For $h=h_{\mu, a}$, we have the following.

Lemma 2.2. For any Borel set $A \subset \mathbb{C}$, we have

$$
M^{h_{\mu, a}}(A) \geq C^{-1} \mu(A)
$$

Proof. Given any $\eta>0$, consider a covering $A \subset \bigcup_{i} B_{i}$ by balls so that

$$
\sum_{i} h_{\mu, a}\left(B_{i}\right) \leq M^{h_{\mu, a}}(A)+\eta
$$

Since $\mu\left(B_{i}\right) \leq C h_{\mu, a}\left(B_{i}\right)$, we have

$$
\mu(A) \leq \sum_{i} \mu\left(B_{i}\right) \leq C \sum_{i} h_{\mu, a}\left(B_{i}\right) \leq C M^{h_{\mu, a}}(A)+C \eta .
$$

Now, for technical reasons we need to extend the function $\varepsilon(\cdot)$ defined on $\mathcal{B}$ to the whole family of bounded sets. Given an arbitrary bounded set $A \subset \mathbb{C}$, let $B$ a ball with minimal diameter that contains $A$. We define $\varepsilon(A):=\varepsilon(B)$. If $B$ is not unique, it does not matter. In this case, for definiteness we can choose the infimum of the values $\varepsilon(B)$ over all balls $B$ with minimal diameter containing $A$, for instance. Analogously, if $h(x, r)=r \varepsilon(x, r)$, we define $h(A)$ as the infimum of the $h(B)$ 's.

It was mentioned above that $\varepsilon_{\mu, a} \in \mathcal{G}_{2}$. Our next objective consists in showing that if $\phi$ is a $K$-quasiconformal planar homeomorphism, then the function defined by

$$
\varepsilon(B)=\varepsilon_{\mu, a}(\phi(B))
$$

for any ball $B \subset \mathbb{C}$, also belongs to $\mathcal{G}_{2}$. In fact, because of the geometric properties of quasiconformal mappings and the smoothness of $\psi_{a}$, it is easily seen that $\varepsilon$ satisfies (2.6). To show that (2.7) also holds requires some more effort. First we need a technical result, whose proof follows from an elementary calculation that we leave for the reader:

Lemma 2.3. Let $a, b>0, a \neq b$, and denote $m=\min (a, b)$. For all $z \in \mathbb{C}$, we have

$$
\sum_{k \geq 0} 2^{-b k} \frac{1}{\left(2^{-k}|z|\right)^{a}+1} \leq \frac{C}{|z|^{m}+1}
$$


with $C$ depending only on $a, b$.

Lemma 2.4. Let $\phi: \mathbb{C} \rightarrow \mathbb{C}$ be a $K$-quasiconformal mapping. If $0<a<C_{1} b$ (where $C_{1}$ is a small positive constant depepending only on $K$ ), then,

$$
\sum_{j \geq 0} \frac{\varepsilon_{\mu, a}\left(\phi\left(B\left(x, 2^{j} r\right)\right)\right)}{2^{b j}} \leq C(K) \varepsilon_{\mu, a}(\phi(B(x, r))) .
$$

In particular, if a is chosen small enough, the function $\varepsilon$ defined by $\varepsilon(B)=\varepsilon_{\mu, a}(\phi(B))$ for any ball $B$, belongs to $\mathcal{G}_{2}$.

Proof. We denote $d_{j}=\operatorname{diam}\left(\phi\left(B\left(x, 2^{j} r\right)\right)\right)$. We have

$$
\begin{aligned}
S & :=\sum_{j \geq 0} \frac{\varepsilon_{\mu, a}\left(\phi\left(B\left(x, 2^{j} r\right)\right)\right)}{2^{b j}} \lesssim \sum_{j \geq 0} \frac{\varepsilon_{\mu, a}\left(B\left(\phi(x), d_{j}\right)\right)}{2^{b j}} \\
& \lesssim \sum_{k \geq 0} \sum_{j: d_{0} 2^{k} \leq d_{j}<d_{0} 2^{k+1}} \frac{\varepsilon_{\mu, a}\left(B\left(\phi(x), 2^{k} d_{0}\right)\right)}{2^{b j}} .
\end{aligned}
$$

For each $j \geq 0$ we have

$$
\frac{d_{j}}{d_{0}}=\prod_{i=1}^{j} \frac{d_{i}}{d_{i-1}} \leq C \prod_{i=1}^{j} \frac{\operatorname{diam}\left(\phi\left(B\left(x, 2^{i} r\right)\right)\right)}{\operatorname{diam}\left(\phi\left(B\left(x, 2^{i-1} r\right)\right)\right)} \leq C(K)^{j}=2^{C_{2} j}
$$

with $C_{2}$ depending on $K$. Thus, for $j, k$ such that $d_{0} 2^{k} \leq d_{j}<d_{0} 2^{k+1}$,

$$
2^{j} \geq\left(\frac{d_{j}}{d_{0}}\right)^{1 / C_{2}} \approx 2^{k / C_{2}}
$$

Then we obtain

$$
S \lesssim \sum_{k \geq 0} \sum_{j: d_{0} 2^{k} \leq d_{j}<d_{0} 2^{k+1}} \frac{\varepsilon_{\mu, a}\left(B\left(\phi(x), 2^{k} d_{0}\right)\right)}{2^{C_{1} b k}} \leq C \sum_{k \geq 0} \frac{\varepsilon_{\mu, a}\left(B\left(\phi(x), 2^{k} d_{0}\right)\right)}{2^{C_{1} b k}}
$$

with $C_{1}=1 / C_{2}$. From Lemma 2.3, if $0<a<C_{1} b$, we infer that

$$
\begin{aligned}
\sum_{k \geq 0} \frac{\varepsilon_{\mu, a}\left(B\left(\phi(x), 2^{k} d_{0}\right)\right)}{2^{C_{1} b k}} & =\sum_{k \geq 0} \frac{1}{2^{\left(1+C_{1} b\right) k} d_{0}} \int \frac{1}{\left(\frac{|\phi(x)-y|}{2^{k} d_{0}}\right)^{1+a}+1} d \mu(y) \\
& \lesssim \frac{1}{d_{0}} \int \frac{1}{\left(\frac{|\phi(x)-y|}{d_{0}}\right)^{1+a}+1} d \mu(y) \\
& =\varepsilon_{\mu, a}\left(B\left(\phi(x), d_{0}\right)\right) \lesssim \varepsilon_{\mu, a}(\phi(B(x, r))) .
\end{aligned}
$$

Another result that shows that some properties of the functions from $\mathcal{G}_{1}$ are preserved under composition with quasiconformal maps is the following. 
Lemma 2.5. Let $\phi: \mathbb{C} \rightarrow \mathbb{C}$ be a $K$-quasiconformal mapping, and $\varepsilon_{0} \in \mathcal{G}_{1}$. Define $\varepsilon(B)=\varepsilon_{0}(\phi(B))$ for any ball $B \subset \mathbb{C}$. For any $s>0$ we have

$$
\int_{0}^{\infty} \varepsilon(x, r)^{s} \frac{d r}{r} \leq C(K, s) \int_{0}^{\infty} \varepsilon_{0}(\phi(x), r)^{s} \frac{d r}{r} .
$$

Proof. We have

$$
\int_{0}^{\infty} \varepsilon_{0}(\phi(B(x, r)))^{s} \frac{d r}{r} \leq C(s) \sum_{j \in \mathbb{Z}} \varepsilon_{0}\left(\phi\left(B\left(x, 2^{j}\right)\right)\right)^{s} .
$$

Denote now $r_{j}=\operatorname{diam}\left(\phi\left(B\left(x, 2^{j}\right)\right)\right.$. We obtain

$$
\begin{aligned}
\sum_{j \in \mathbb{Z}} \varepsilon_{0}\left(\phi\left(B\left(x, 2^{j}\right)\right)^{s}\right. & =\sum_{k \in \mathbb{Z}} \sum_{j: 2^{k} \leq r_{j}<2^{k+1}} \varepsilon_{0}\left(\phi\left(B\left(x, 2^{j}\right)\right)^{s}\right. \\
& \lesssim \sum_{k \in \mathbb{Z}} \sum_{j: 2^{k} \leq r_{j}<2^{k+1}} \varepsilon_{0}\left(B\left(\phi(x), r_{j}\right)\right)^{s} \\
& \lesssim C(K) \sum_{k \in \mathbb{Z}} \varepsilon_{0}\left(B\left(\phi(x), 2^{k}\right)\right)^{s} \leq C(K, s) \int_{0}^{\infty} \varepsilon_{0}(\phi(x), r)^{s} \frac{d r}{r}
\end{aligned}
$$

where we took into account that $\#\left\{j: 2^{k} \leq r_{j}<2^{k+1}\right\} \leq C(K)$ because of the geometric properties of quasiconformal mappings.

2.3. The space $\operatorname{Lip}^{q}(\varepsilon)$. We wish to obtain distortion estimates by quasiconformal maps in terms of the contents $M^{h}$, from which we will derive the corresponding estimates in terms of non linear potentials. To study the distortion in terms of $M^{h}$, our arguments below are inspired by the ones of $\left[\mathrm{ACM}^{+} 08\right]$.

Given $1 \leq q<\infty$ and a function $\varepsilon: \mathcal{B} \rightarrow[0, \infty)$, we define $\operatorname{Lip}^{q}(\varepsilon)$ as the class of all functions $f: \mathbb{C} \rightarrow \mathbb{C}$ for which there is some constant $M$ such that

$$
\left(\frac{1}{|B|} \int_{B}\left|f-f_{B}\right|^{q}\right)^{1 / q} \leq M \varepsilon(B)
$$

for all balls $B$. In the sufficient part of the definition, one can replace the average $f_{B}=|B|^{-1} \int_{B} f$ by any constant $c_{B}$, getting the same class of functions. The infimum of all these constants $M$ is denoted by $\|f\|_{\operatorname{Lip}^{q}(\varepsilon)}$.

Let us look at the behaviour of a function in $\operatorname{Lip}(\varepsilon)=\operatorname{Lip}^{1}(\varepsilon)$ under a $K$-quasiconformal mapping.

Lemma 2.6. Let $\varepsilon_{0} \in \mathcal{G}_{1}$ and let $\phi: \mathbb{C} \rightarrow \mathbb{C}$ be a $K$-quasiconformal mapping. Set

$$
\varepsilon(B):=\varepsilon_{0}(\phi(B))
$$

with $a>0$, and $h(x, r)=r \varepsilon(x, r)$. Then, given $q$ with $K<q<\infty$, for all $f \in \operatorname{Lip}^{q}\left(\varepsilon_{0}\right)$, we have $f \circ \phi \in \operatorname{Lip}(\varepsilon)$ and

$$
\|f \circ \phi\|_{\operatorname{Lip}(\varepsilon)} \leq C(q, K)\|f\|_{\operatorname{Lip}^{q}\left(\varepsilon_{0}\right)} .
$$


Proof. We will follow the techniques used in Rei74. Given a ball $B=B(x, t)$, we can find a ball $B_{0}$ centered at $\phi(x)$ such that $B_{0} \supset \phi(B)$ and $|B| \leq\left|\phi^{-1}\left(B_{0}\right)\right| \leq$ $C(K)|B|$, where $C(K)$ depends only on $K$. We have:

$$
\begin{aligned}
& \frac{1}{|B|} \int_{B}\left|f \circ \phi(z)-c_{B}\right| d m(z)=\frac{1}{|B|} \int_{\phi(B)}\left|f(w)-c_{B}\right| J \phi^{-1}(w) d m(w) \\
& \quad \leq \frac{1}{|B|} \int_{B_{0}}\left|f(w)-c_{B}\right| J \phi^{-1}(w) d m(w) \\
& \quad \leq C(K) \frac{1}{\left|B_{0}\right|} \int_{B_{0}}\left|f(w)-c_{B}\right| J \phi^{-1}(w) d m(w) \frac{\left|B_{0}\right|}{\left|\phi^{-1}\left(B_{0}\right)\right|} \\
& \quad \leq C(K)\left(\frac{1}{\left|B_{0}\right|} \int_{B_{0}}\left|f(w)-c_{B}\right|^{q} d m(w)\right)^{1 / q} \frac{\left(\frac{1}{\left|B_{0}\right|} \int_{B_{0}} J \phi^{-1}(w)^{q^{\prime}} d m(w)\right)^{1 / q^{\prime}}}{\left(\frac{1}{\left|B_{0}\right|} \int_{B_{0}} J \phi^{-1}(w) d m(w)\right)} \\
& \quad \leq C(K, q)\|f\|_{\operatorname{Lip}^{q}\left(\varepsilon_{0}\right)} \varepsilon_{0}\left(B_{0}\right),
\end{aligned}
$$

where the last inequality follows from the fact that the Jacobian satisfies the reverse Hölder inequality

$$
\left(\frac{1}{\left|B_{0}\right|} \int_{B_{0}} J \phi^{-1}(w)^{q^{\prime}} d m(w)\right)^{1 / q^{\prime}} \leq C(K, q) \frac{1}{\left|B_{0}\right|} \int_{B_{0}} J \phi^{-1}(w) d m(w)
$$

for $q^{\prime}<K /(K-1)$, by [AIS01, p.37].

Since $\varepsilon_{0} \in \mathcal{G}_{1}$, we have $\varepsilon_{0}\left(B_{0}\right) \approx \varepsilon_{0}(\phi(B))=\varepsilon(B)$, and then

$$
\frac{1}{|B|} \int_{B}\left|f \circ \phi(z)-c_{B}\right| d m(z) \leq C(K, q)\|f\|_{\operatorname{Lip}^{q}\left(\varepsilon_{0}\right)} \varepsilon(B) .
$$

Thus $\|f \circ \phi\|_{L i p(\varepsilon)} \leq C(K, q)\|f\|_{L^{\prime p^{q}}\left(\varepsilon_{0}\right)}$.

2.4. The capacities $\gamma_{h, q}$. Given $1<q<\infty$, for a bounded set $F \subset \mathbb{C}$ and a function $h: \mathcal{B} \rightarrow[0, \infty)$, with $h(x, r)=r \varepsilon(x, r)$, we set

$$
\gamma_{h, q}(F)=\sup |\langle\bar{\partial} f, 1\rangle|=\sup \left|f^{\prime}(\infty)\right|
$$

where the supremum is taken over all $\operatorname{Lip}^{q}(\varepsilon)$ functions with $\|f\|_{\operatorname{Lip}^{q}(\varepsilon)} \leq 1, f(\infty)=0$ and such that $\bar{\partial} f$ is a distribution supported on $F$.

Lemma 2.7. Let $E$ be a compact set and $\varepsilon \in \mathcal{G}_{1}$. For $1 \leq q<2$ we have

(a) $\gamma_{h, q}(E) \leq C M^{h}(E)$.

(b) If moreover $\varepsilon \in \mathcal{G}_{2}$, then $M^{h}(E) \leq C(q) \gamma_{h, q}(E)$.

Proof. First we show (a). Fix a real number $\eta>0$ and take a covering of $E$ by balls $B_{j}$, with radius $r_{j}$, such that $\sum_{j} h\left(B_{j}\right) \leq M^{h}(E)+\eta$. Consider a partition of unity associated to this covering, that is, for each $j$ we take an infinitely differentiable 
function $\varphi_{j}$ supported on $2 B_{j}$ with $\left\|\nabla \varphi_{j}\right\|_{\infty} \leq \frac{C}{r_{j}}$, and so that $\sum_{j} \varphi_{j}=1$ on a neighbourhood of $E$. Then, if $\|f\|_{\operatorname{Lip}^{q}(\varepsilon)} \leq 1$,

$$
\begin{aligned}
|\langle\bar{\partial} f, 1\rangle| & =\left|\left\langle\bar{\partial} f, \sum_{j} \varphi_{j}\right\rangle\right|=\left|\sum_{j}\left\langle\bar{\partial}\left(f-f_{2 B_{j}}\right), \varphi_{j}\right\rangle\right| \\
& \leq \sum_{j} \int_{2 B_{j}}\left|f-f_{2 B_{j}}\right|\left|\bar{\partial} \varphi_{j}\right| d m \leq \sum_{j} \frac{C}{r_{j}} \int_{2 B_{j}}\left|f-f_{2 B_{j}}\right| d m \\
& \leq C \sum_{j} r_{j} \varepsilon\left(2 B_{j}\right) \leq C \sum_{j} r_{j} \varepsilon\left(B_{j}\right) \leq C\left(M^{h}(E)+\eta\right) .
\end{aligned}
$$

Hence, $\gamma_{h, q}(E) \leq C M^{h}(E)$.

To prove (b), we suppose that $\varepsilon \in \mathcal{G}_{2}$. If $M^{h}(E)>0$ then by Frostman's Lemma there exists a positive measure $\nu$, supported on $E$, such that $\nu(B(x, r)) \leq h(x, r)$ and $\nu(E) \geq C M^{h}(E)$. The function $f=\nu * \frac{1}{z}$ is analytic outside $E, f(\infty)=0$ and $\langle\bar{\partial} f, 1\rangle=\nu(E)$. Now, we will check that $f \in \operatorname{Lip}^{q}(\varepsilon)$. Fix a ball $B=B\left(z_{0}, r\right)$ and $c_{B}=\int_{\mathbb{C} \backslash 2 B} \frac{d \nu(w)}{w-z_{0}}$. We have

$$
\begin{aligned}
& \frac{1}{|B|} \int_{B}\left|f(z)-c_{B}\right|^{q} d m(z) \leq \\
& \quad \leq \frac{1}{|B|} \int_{B}\left(\int_{2 B} \frac{1}{|w-z|} d \nu(w)+\int_{\mathbb{C} \backslash 2 B}\left|\frac{1}{w-z}-\frac{1}{w-z_{0}}\right| d \nu(w)\right)^{q} d m(z) .
\end{aligned}
$$

For the first term on the right side, by Hölder's inequality and Fubini's Theorem, since $q<2$,

$$
\begin{aligned}
\frac{1}{|B|} \int_{B}\left(\int_{2 B} \frac{1}{|w-z|} d \nu(w)\right)^{q} d m(z) & \leq \frac{\nu(2 B)^{q-1}}{|B|} \int_{2 B} \int_{B} \frac{1}{|w-z|^{q}} d m(z) d \nu(w) \\
& \leq C \frac{\nu(2 B)^{q}}{r^{q}} \leq C \varepsilon(2 B)^{q} \leq C \varepsilon(B)^{q} .
\end{aligned}
$$

Since $|w-z| \simeq\left|w-z_{0}\right|$ for $z \in B$ and $w \in \mathbb{C} \backslash 2 B$, we have

$$
\begin{aligned}
\int_{\mathbb{C} \backslash 2 B} \frac{\left|z-z_{0}\right|}{\left|w-z_{0}\right|^{2}} d \nu(w) & \leq C r \sum_{j=1}^{\infty} \int_{2^{j+1} B \backslash 2^{j} B} \frac{d \nu(w)}{\left|w-z_{0}\right|^{2}} \\
& \leq C r \sum_{j=1}^{\infty} \frac{h\left(z_{0}, 2^{j+1} r\right)}{\left(2^{j} r\right)^{2}} \leq C \sum_{j=0}^{\infty} \frac{\varepsilon\left(2^{j} B\right)}{2^{j}} \leq C \varepsilon(B),
\end{aligned}
$$

using the fact that $\varepsilon \in \mathcal{G}_{2}$. Thus we get

$$
\frac{1}{|B|} \int_{B}\left(\int_{\mathbb{C} \backslash 2 B}\left|\frac{1}{w-z}-\frac{1}{w-z_{0}}\right| d \nu(w)\right)^{q} d m(z) \leq C \varepsilon(B)^{q},
$$

and so (b) follows. 
Recall that a quasiconformal mapping $\phi: \mathbb{C} \rightarrow \mathbb{C}$ is called principal if it is conformal outside a compact set and $|\phi(z)-z|=O(1 /|z|)$ as $z \rightarrow \infty$.

Lemma 2.8. Let $E$ be a compact set, and $\phi: \mathbb{C} \rightarrow \mathbb{C}$ a principal $K$-quasiconformal mapping, conformal on $\mathbb{C} \backslash E$. Given $\varepsilon_{0} \in \mathcal{G}_{1}$, define

$$
\varepsilon(x, r)=\varepsilon_{0}(\phi(B(x, r))
$$

and $h(x, t)=r \varepsilon(x, r)$. For $q>K$, we have

$$
\gamma_{h_{0}, q}(\phi(E)) \leq C \gamma_{h, 1}(E)
$$

Proof. Consider $f \in \operatorname{Lip}^{q}\left(\varepsilon_{0}\right)$ which is analytic in $\mathbb{C} \backslash \phi(E),\|f\|_{\operatorname{Lip}^{q}\left(\varepsilon_{0}\right)} \leq 1$ and $f(\infty)=0$. Set $g=f \circ \phi$. Then $g$ is analytic on $\mathbb{C} \backslash E$ and, by Lemma 2.6, $g \in \operatorname{Lip}^{1}(\varepsilon)$ and $\|g\|_{\operatorname{Lip}^{1}(\varepsilon)} \leq C(K)\|f\|_{\operatorname{Lip}^{q}\left(\varepsilon_{0}\right)} \leq C(K)$. So, we have $\left|g^{\prime}(\infty)\right| \leq C(K) \gamma_{h, 1}(E)$. Moreover, since $\phi$ is principal, $\phi^{\prime}(\infty)=1$ and so

$$
\left|g^{\prime}(\infty)\right|=\left|f^{\prime}(\infty)\right|\left|\phi^{\prime}(\infty)\right|=\left|f^{\prime}(\infty)\right|
$$

Consequently $\gamma_{h_{0}, q}(\phi(E)) \leq C(K) \gamma_{h, 1}(E)$.

2.5. Distortion of $h$-contents. From Lemmas 2.7 and 2.8 we get:

Lemma 2.9. Let $E$ be a compact set, and $\phi: \mathbb{C} \rightarrow \mathbb{C}$ a principal $K$-quasiconformal mapping, with $K<2$, conformal on $\mathbb{C} \backslash E$. Given $\varepsilon_{0} \in \mathcal{G}_{2}$, define

$$
\varepsilon(x, r)=\varepsilon_{0}\left(\phi^{-1}(B(x, r))\right.
$$

and $h(x, r)=r \varepsilon(x, r)$. We have

$$
M^{h_{0}}(E) \leq C M^{h}(\phi(E))
$$

Proof. Take $q$ such that $K<q<2$. By Lemma 2.7, we have $M^{h_{0}}(E) \leq C \gamma_{h_{0}, q}(E)$. By Lemma 2.8 (applied to $\left.\phi^{-1}\right), \gamma_{h_{0}, q}(E) \leq C \gamma_{h, 1}(\phi(E))$. Finally, by Lemma 2.7] again, $\gamma_{h, 1}(\phi(E)) \leq C M^{h}(\phi(E))$.

Our next objective in this section is to extend Lemma 2.9 to the case $K \geq 2$.

Lemma 2.10. Let $\varepsilon: \mathcal{B} \rightarrow[0, \infty)$ be a function from $\mathcal{G}_{1}$, and set $h(x, r)=r \varepsilon(x, r)$. Suppose that for any principal $K_{0}$-quasiconformal mapping $\phi: \mathbb{C} \rightarrow \mathbb{C}$ conformal on $\mathbb{C} \backslash E$, with $E$ compact, and with $K_{0} \leq K$, the function $\varepsilon_{\phi}: \mathcal{B} \rightarrow[0, \infty)$ defined by $\varepsilon_{\phi}(B)=\varepsilon\left(\phi^{-1}(B)\right)$ is in $\mathcal{G}_{2}$. Then,

$$
M^{h}(E) \leq C(K) M^{h_{\phi}}(\phi(E))
$$

for any compact set $E \subset \mathbb{C}$, where $h_{\phi}(x, r)=r \varepsilon_{\phi}(x, r)$.

Proof. We factorize $\phi$ so that $\phi=\phi_{n} \circ \cdots \circ \phi_{1}$, where $\phi_{i}$ are $K^{1 / n}$-quasiconformal mappings conformal on $\mathbb{C} \backslash \phi_{n-1}(E)$, with $n$ big enough so that $K^{1 / n}<2$. So we have

$$
E=E_{0} \stackrel{\phi_{1}}{\longrightarrow} E_{1} \stackrel{\phi_{2}}{\longrightarrow} \ldots \stackrel{\phi_{n-1}}{\longrightarrow} E_{n-1} \stackrel{\phi_{n}}{\longrightarrow} E_{n}=\phi(E)
$$


By Lemma 2.9, we have

$$
M^{h}(E)=M^{h}\left(E_{0}\right) \leq C M^{h_{1}}\left(\phi_{1}\left(E_{0}\right)\right)=C M^{h_{1}}\left(E_{1}\right),
$$

where $\varepsilon_{1}(B)=\varepsilon\left(\phi_{1}^{-1}(B)\right)$ (notice that $\varepsilon \in \mathcal{G}_{2}$ by hypothesis).

Denote now $\varepsilon_{2}(B)=\varepsilon_{1}\left(\phi_{2}^{-1}(B)\right)=\varepsilon\left(\phi_{1}^{-1}\left(\phi_{2}^{-1}(B)\right)\right)$ and $h_{2}(x, r)=r \varepsilon_{2}(x, r)$. Since $\varepsilon_{1} \in \mathcal{G}_{2}$ by the hypotheses above, by Lemma 2.9 again,

$$
M^{h_{1}}\left(E_{1}\right) \leq C M^{h_{2}}\left(E_{2}\right) .
$$

Going on in this way, after $n$ steps we obtain

$$
M^{h}\left(E_{0}\right) \leq C M^{h_{1}}\left(E_{1}\right) \leq \cdots \leq C M^{h_{n}}\left(E_{n}\right),
$$

with $E=E_{0}, \quad E_{n}=\phi(E), h_{n}(x, r)=r \varepsilon_{n}(x, r)$, and

$$
\varepsilon_{n}(B)=\varepsilon\left(\phi_{1}^{-1}\left(\phi_{2}^{-1}\left(\cdots \phi_{n}^{-1}(E)\right)\right)\right) \approx \varepsilon\left(\phi^{-1}(B)\right)=\varepsilon_{\phi}(B) .
$$

2.6. Proof of Theorem 1.2 (b). Recall that $\mu$ is a Borel measure supported on $E$ such that $\dot{W}_{1 / p, p}^{\mu}(x) \leq 1$ for all $x \in \mathbb{C}$ and such that $\dot{C}_{1 / p, p}(E) \approx \mu(E)$. We know that $M^{h_{\mu, a}}(E) \geq C^{-1} \mu(E)$, with $h_{\mu, a}$ defined in (2.4) and a small enough. Set

$$
\varepsilon(x, r)=\varepsilon_{\mu, a}\left(\phi^{-1}(B(x, r))\right)
$$

and $h(x, r)=r \varepsilon(x, r)$. By Lemma 2.4, $\varepsilon \circ \phi^{-1} \in \mathcal{G}_{2}$ for any $K_{0}$-quasiconformal mapping such that $K_{0} \leq K$. Then, by Lemma 2.10 we have

$$
M^{h_{\mu, a}}(E) \leq C M^{h}(\phi(E)) .
$$

By Frostman's Lemma there exists some measure $\nu$ supported on $\phi(E)$ such that $\nu(\phi(E)) \geq C^{-1} M^{h}(\phi(E))$ with $\nu(B) \leq h(B)$ for all balls $B$. Recall that

$$
\int_{0}^{\infty} \varepsilon_{\mu, a}(x, r)^{p^{\prime}-1} \frac{d r}{r} \lesssim 1 \quad \text { for all } x \in \mathbb{C},
$$

by (2.5). From Lemma 2.5 we deduce that this also holds with $\varepsilon$ instead of $\varepsilon_{\mu, a}$, and thus

$$
\int_{0}^{\infty}\left(\frac{\nu(B(x, r))}{r}\right)^{p^{\prime}-1} \frac{d r}{r} \leq \int_{0}^{\infty}\left(\frac{h(x, r)}{r}\right)^{p^{\prime}-1} \frac{d r}{r} \leq C .
$$

In terms of Wolff's potentials, this is the same as saying that

$$
\dot{W}_{1 / p, p}^{\nu}(x) \leq C
$$

for all $x \in \mathbb{C}$. Therefore,

$$
\dot{C}_{1 / p, p}(\phi(E)) \gtrsim \nu(\phi(E)) \gtrsim M^{h}(\phi(E)) \gtrsim M^{h_{\mu, a}}(E) \gtrsim \mu(E) \gtrsim \dot{C}_{1 / p, p}(E) .
$$




\subsection{The main lemma on $h$-contents.}

Lemma 2.11. Let $\varepsilon_{0} \in \mathcal{G}_{1}$ and set $h_{0}(x, r)=r \varepsilon_{0}(x, r)$. Suppose that for any $K_{0^{-}}$ quasiconformal mapping $\psi: \mathbb{C} \rightarrow \mathbb{C}$, with $K_{0} \leq K$, we have $\varepsilon_{0} \circ \psi \in \mathcal{G}_{2}$. Let $E \subset B(0,1 / 2)$ be compact and $\phi: \mathbb{C} \rightarrow \mathbb{C}$ a principal $K$-quasiconformal mapping, conformal on $\mathbb{C} \backslash \overline{\mathbb{D}}$. Denote

$$
\varepsilon(x, r):=\varepsilon_{0}\left(\phi^{-1}(B(x, r))\right)^{2 K /(K+1)}, \quad h(x, r):=r^{2 /(K+1)} \varepsilon(x, r) .
$$

Then we have

$$
M^{h_{0}}(E) \leq C(K) M^{h}(\phi(E))^{(K+1) / 2 K} .
$$

Proof. Consider an arbitrary covering $\phi(E) \subset \bigcup_{i} B_{i}$ by a finite number of balls $B_{i}:=B\left(x_{i}, t_{i}\right)$ (recall that $\phi(E)$ is compact). For each $i$, take also a ball $D_{i}$ centered at $\phi^{-1}\left(x_{i}\right)$ which contains $\phi^{-1}\left(B_{i}\right)$ and which has comparable diameter.

We denote $\Omega=\bigcup_{i} D_{i}$. Notice that $E \subset \Omega$. Then we consider the decomposition $\phi=\phi_{2} \circ \phi_{1}$, where $\phi_{1}, \phi_{2}$ are principal $K$-quasiconformal mappings. Moreover, we require $\phi_{1}$ to be conformal outside $\bar{\Omega}$ and $\phi_{2}$ conformal in $\phi_{1}(\Omega) \cup(\mathbb{C} \backslash \mathbb{D})$.

By Lemma 2.10, we have

$$
M^{h_{0}}(E) \leq M^{h_{0}}(\Omega) \leq C M^{\widetilde{h}}\left(\phi_{1}(\Omega)\right)
$$

with

$$
\widetilde{h}(x, r)=r \widetilde{\varepsilon}(x, r):=r \varepsilon_{0}\left(\phi_{1}^{-1}(B(x, r))\right) .
$$

Now we will estimate $M^{\widetilde{h}}\left(\phi_{1}(\Omega)\right)$ in terms of $M^{h}(\phi(E))$. For each $i$, let $\widetilde{D}_{i}$ be a ball centered at $\phi_{2}^{-1}\left(x_{i}\right)$ containing $\phi_{1}\left(D_{i}\right)$ and such that $\operatorname{diam}\left(\widetilde{D}_{i}\right) \approx \operatorname{diam}\left(\phi_{1}\left(D_{i}\right)\right)$. Notice that we also have

$$
B_{i} \subset \phi_{2}\left(\widetilde{D}_{i}\right) \quad \text { and } \quad \operatorname{diam}\left(B_{i}\right) \approx \operatorname{diam}\left(\phi_{2}\left(\widetilde{D}_{i}\right)\right)
$$

By Vitali's covering lemma there exists a subfamily of disjoint balls $\widetilde{D}_{j}, j \in J$, such that $\phi_{1}(E) \subset \cup_{j} 5 \widetilde{D}_{j}$. Applying Hölder's inequality twice (once for the intergral and once for the sum), the fact that $J\left(\phi_{2}^{-1}\right) \in L^{K /(K-1)}(\phi(\Omega))$ because of the improved borderline integrability of the Jacobian $J\left(\phi_{2}^{-1}\right)$ under the assumption that $\phi_{2}: \phi_{1}(\Omega) \rightarrow \phi(\Omega)$ is conformal (by [AN03, Lemma 5.2]), and (2.10) (these last two 
facts yield the last inequality), we obtain

$$
\begin{aligned}
M^{\widetilde{h}}\left(\phi_{1}(\Omega)\right) \leq & \sum_{j \in J} \widetilde{h}\left(5 \widetilde{D}_{j}\right) \leq C \sum_{j \in J} \widetilde{h}\left(\widetilde{D}_{j}\right)=C \sum_{j \in J} \operatorname{diam}\left(\phi_{1}\left(D_{j}\right)\right) \widetilde{\varepsilon}\left(\widetilde{D}_{j}\right) \\
\leq & C \sum_{j \in J}\left(\int_{\phi\left(D_{j}\right)} J\left(\phi_{2}^{-1}\right) d m\right)^{1 / 2} \widetilde{\varepsilon}\left(\widetilde{D}_{j}\right) \\
\leq & C \sum_{j \in J}\left(\int_{\phi\left(D_{j}\right)} J\left(\phi_{2}^{-1}\right)^{K /(K-1)} d m\right)^{(K-1) / 2 K} \operatorname{diam}\left(\phi\left(D_{j}\right)\right)^{1 / K} \widetilde{\varepsilon}\left(\widetilde{D}_{j}\right) \\
\leq & C\left(\sum_{j \in J} \int_{\phi\left(D_{j}\right)} J\left(\phi_{2}^{-1}\right)^{K /(K-1)} d m\right)^{(K-1) / 2 K} \\
& \times\left(\sum_{j \in J} \operatorname{diam}\left(\phi\left(D_{j}\right)\right)^{2 /(K+1)} \widetilde{\varepsilon}\left(\widetilde{D}_{j}\right)^{2 K /(K+1)}\right)^{(K+1) / 2 K} \\
\leq & C\left(\sum_{j \in J} \operatorname{diam}\left(B_{j}\right)^{2 /(K+1)} \widetilde{\varepsilon}\left(\widetilde{D}_{j}\right)^{2 K /(K+1)}\right)^{(K+1) / 2 K} .
\end{aligned}
$$

Notice now that

$$
\widetilde{\varepsilon}\left(\widetilde{D}_{j}\right)=\varepsilon_{0}\left(\phi_{1}^{-1}\left(\widetilde{D}_{j}\right)\right) \approx \varepsilon_{0}\left(D_{j}\right) \approx \varepsilon_{0}\left(\phi^{-1}\left(B_{j}\right)\right) .
$$

Recalling that

$$
\varepsilon(x, t):=\varepsilon_{0}\left(\phi^{-1}(B(x, t))\right)^{2 K /(K+1)} \quad \text { and } \quad h(x, t)=t^{2 /(K+1)} \varepsilon(x, t),
$$

we deduce

$$
M^{h_{0}}(E) \leq C M^{\tilde{h}}\left(\phi_{1}(\Omega)\right) \leq C\left(\sum_{j \in J} h\left(B_{j}\right)\right)^{(K+1) / 2 K} .
$$

If we take the infimum over all coverings of $\phi(E)$ by balls $B_{j}$, then we get

$$
M^{h_{0}}(E) \leq C M^{h}(\phi(E))^{\frac{K+1}{2 K}} .
$$

2.8. Proof of Theorem 1.2 (a). By standard methods (see e.g. [LSUT10], p.289, proof of lemma 2.1), we may assume that $\phi$ is a principal quasiconformal mapping, conformal on $\mathbb{C} \backslash \overline{\mathbb{D}}$, and that $E \subset B(0,1 / 2)=: \frac{1}{2} B$ (and so $\operatorname{diam}(\phi(B)) \approx 1$ ).

Let $\mu$ be a Borel measure supported on $E$ such that $\dot{W}_{1 / p, p}^{\mu}(x) \leq 1$ for all $x \in \mathbb{C}$ and such that $\dot{C}_{1 / p, p}(E) \approx \mu(E)$. We know that $M^{h_{\mu, a}}(E) \geq C^{-1} \mu(E)$. If $0<a<1$ is small enough, then $\varepsilon_{0}:=\varepsilon_{\mu, a}$ satisfies the assumptions of Lemma 2.11, and so

$$
M^{h_{\mu, a}}(E) \leq C(K) M^{h}(\phi(E))^{(K+1) / 2 K},
$$


with $h$ given by (2.9) (replacing $\varepsilon_{0}$ there by $\varepsilon_{\mu, a}$ ). By the definition of $\varepsilon$ and Lemma 2.5,

$$
\begin{aligned}
\int_{0}^{\infty} \varepsilon(x, r)^{\frac{\left(p^{\prime}-1\right)(K+1)}{2 K} \frac{d r}{r}} & =\int_{0}^{\infty} \varepsilon_{\mu, a}\left(\phi^{-1}(B(x, r))\right)^{p^{\prime}-1} \frac{d r}{r} \\
& \leq C \int_{0}^{\infty} \varepsilon_{\mu, a}\left(\phi^{-1}(x), r\right)^{p^{\prime}-1} \frac{d r}{r} \leq C
\end{aligned}
$$

for all $x \in \mathbb{C}$.

Now we apply Frostman's lemma again, and we deduce that there exists some measure $\nu$ supported on $\phi(E)$ such that $\nu(\phi(E)) \geq C^{-1} M^{h}(\phi(E))$ with $\nu(B) \leq h(B)$ for all balls $B$. So we have

$$
\int_{0}^{\infty}\left(\frac{\nu(B(x, r))}{r^{\frac{2}{K+1}}}\right)^{\frac{\left(p^{\prime}-1\right)(K+1)}{2 K}} \frac{d r}{r} \leq C
$$

for all $x \in \mathbb{C}$. In terms of Wolff's potentials, this is the same as saying that

$$
\dot{W}_{\alpha, q}^{\nu}(x) \leq C
$$

for all $x \in \mathbb{C}$, with

$$
\alpha=\frac{2 K}{2 K p-K+1}, \quad q=\frac{2 K p-K+1}{K+1} .
$$

Therefore,

$$
\dot{C}_{\alpha, q}(\phi(E)) \gtrsim \nu(\phi(E)) \gtrsim \dot{C}_{1 / p, p}(E)^{\frac{2 K}{K+1}}
$$

\section{Strategy for the Proof of Theorem 1.1}

Sections 3-7 are devoted to the proof of Theorem 1.1. An equivalent way of formulating this theorem consists in saying that if $E \subset B(0,1 / 2)$ is compact and $\phi: \mathbb{C} \rightarrow \mathbb{C}$ a principal $K$-quasiconformal mapping, conformal on $\mathbb{C} \backslash \bar{B}(0,1)$, then

$$
\dot{C}_{\frac{2 K}{2 K+1}, \frac{2 K+1}{K+1}}(\phi(E)) \geq c^{-1} \gamma(E)^{\frac{2 K}{K+1}},
$$

by appropriate normalizations. To prove this result we will use the following tools:

- the characterization of analytic capacity in terms of curvature in Theorem A,

- a corona type decomposition for measures with finite curvature analogous to the one used in [Tol05] to study the behavior of analytic capacity under bilipschitz maps,

- the main Lemma 2.11 on the distortion of $h$-contents under quasiconformal maps,

- improved quantitative estimates for the distortion of sub-arcs of chord arc curves. 
Let us describe the arguments to prove (3.1) in more detail. Given, $E \subset \mathbb{C}$ with $\gamma(E)>0$, let $\mu$ be a measure supported on $E$ such that $\mu(E) \approx \gamma(E), \mu(B(x, r)) \leq r$ for all $x \in \mathbb{C}, r>0$, and $c_{\mu}^{2}(x) \leq 1$ for all $x \in \mathbb{C}$. As in the preceding section, for each $a>0$ we construct the measure $\mathcal{H}^{h_{a}}$ associated to $\mu$, with $h_{a}(x, t)=t \varepsilon_{a}(x, t)$, where

$$
\varepsilon_{a}(x, t)=\frac{1}{t} \int \psi_{a}\left(\frac{y-x}{t}\right) d \mu(y)
$$

and $\psi_{a}$ is defined as in (2.2). To simplify the notation, now we will write $\varepsilon_{a}$ and $h_{a}$ instead of $\varepsilon_{\mu, a}$ and $h_{\mu, a}$. The main Lemma 2.11 on the distortion of $h$-contents tells us that

$$
M^{h}(\phi(E)) \gtrsim M^{h_{a}}(E)^{\frac{2 K}{K+1}} \gtrsim \mu(E)^{\frac{2 K}{K+1}},
$$

where $h$ is the gauge function defined by

$$
h(x, t):=t^{2 /(K+1)} \varepsilon(x, t), \quad \varepsilon(x, t):=\varepsilon_{a}\left(\phi^{-1}(B(x, t))\right)^{2 K /(K+1)},
$$

with $a>0$ small enough.

By Frostman's Lemma we deduce that there exists a measure $\nu$ supported on $\phi(E)$ satisfying $\nu(\phi(E)) \approx M^{h}(\phi(E))$ and $\nu(B(x, r)) \leq h(x, t)$. However, from the last estimate we cannot infer that

$$
\dot{W}_{\frac{2 K}{2 K+1}}^{\nu}, \frac{2 K+1}{K+1}(x) \leq C \quad \text { for all } x \in \mathbb{C},
$$

as in the proof of Theorem 1.2 , because now the estimate

$$
\dot{W}_{2 / 3,3 / 2}^{\mu}(x) \leq C \quad \text { for all } x \in \mathbb{C}
$$

may be false.

To obtain a measure $\nu$ supported on $\phi(E)$ satisfying (3.2) we will use the information on the curvature of $\mu$. Indeed, by [Tol05, Main Lemma 3.1], there exists some collection of squares $\operatorname{Top}(\mu)$ such that

$$
\sum_{Q \in \operatorname{Top}(\mu)} \theta_{\mu}(Q)^{2} \mu(Q) \leq C\left(\mu(E)+\int c_{\mu}^{2}(x) d \mu(x)\right) \leq C \mu(E),
$$

where $\theta_{\mu}(Q)=\mu(Q) / \ell(Q)$ (here $\ell(Q)$ stands for the side length of $Q$ ), and the last inequality is a consequence of the normalization $c_{\mu}^{2}(x) \leq 1$ for all $x \in \mathbb{C}$. For each square $Q \in \operatorname{Top}(\mu)$ there exists some chord arc curve $\Gamma_{Q}$ (or a fixed finite number of chord arc curves) satisfying some precise properties. Roughly speaking, if a dyadic square $P$ intersects $E$ and $\ell(P) \leq \operatorname{diam}(E)$, then it belongs to some "tree" with "root" $Q \in \operatorname{Top}(\mu)$ and $P$ is close to the curve $\Gamma_{Q}$. For more precise information, see [Tol05].

It is easy to check (but we will not need it for the proof, see however Lemma 5.10 and Section 6 which contains the actual proof), that (3.3) implies that

$$
\sum_{Q \in \operatorname{Top}(\mu)} \varepsilon_{a}(Q)^{2} \mu(Q) \leq C \mu(E)
$$


By Tchebytchev, we infer that for all $x$ in a subset $E_{0} \subset E$ with $\mu\left(E_{0}\right) \geq \mu(E) / 2$,

$$
\sum_{Q \in \operatorname{Top}(\mu): x \in Q} \varepsilon_{a}(Q)^{2} \leq C .
$$

Arguing as in the preceding section (but again we will not need this for the proof, Section 6 contains the actual proof), this implies that

$$
\sum_{Q \in \phi(\operatorname{Top}(\mu)): x \in Q}\left(\frac{h(Q)}{\ell(Q)^{2 /(K+1)}}\right)^{\frac{K+1}{K}} \leq C
$$

for all $x \in \phi\left(E_{0}\right)$. By Frostman's Lemma, we deduce that there exists a measure $\nu$ supported on $\phi(E)$ with $\nu(2 Q) \leq h(2 Q) \lesssim h(Q)$ for all the squares $Q$, and so

$$
\sum_{Q \in \phi(\operatorname{Top}(\mu)): x \in Q}\left(\frac{\nu(2 Q)}{\ell(Q)^{2 /(K+1)}}\right)^{\frac{K+1}{K}} \leq C .
$$

In this inequality, if instead of summing over all the squares $Q \in \phi(\operatorname{Top}(\mu))$ containing $x$ we summed over all $Q \in \phi(\mathcal{D})$ containing $x$, then we would obtain $(3.2)$, and thus

$$
\dot{C}_{\frac{2 K}{2 K+1}, \frac{2 K+1}{K+1}}(\phi(E)) \gtrsim \nu(\phi(E)) \gtrsim \mu\left(E_{0}\right)^{\frac{2 K}{K+1}} \gtrsim \gamma(E)^{\frac{2 K}{K+1}}
$$

In a sense, to extend the sum in (3.4) from the squares in $\phi($ Top) to the entire collection of $Q \in \phi(\mathcal{D})$, we can use the geometric properties of the corona decomposition (i.e. different scales). To be able to use this information, we have to obtain improved distortion estimates for subsets of chord arc curves, in a more quantitative way than the ones of $\left[\mathrm{ACM}^{+} 08\right.$, Section 3] for rectifiable sets. This is what we do in next section.

To tell the truth, in the arguments above, when we apply Tchebytchev to obtain the subset $E_{0} \subset E$, some of the delicate properties of the corona decomposition for $\mu$ are destroyed, and so we will follow a somewhat different approach, although similar in spirit to the one outlined above. Because of this reason, we will need to obtain a corona decomposition for $\mu$ slightly different to the one in [Tol05]. We carry out this task in Section 5. The required measure $\nu$ is constructed in Section 6. A direct application of Frostman Lemma is not enough, and we will have to use a more sophisticated argument more adapted to the corona decomposition. Finally, in Section 7 we prove that the key estimate (3.2) holds for $\nu$.

\section{Distortion of SUB-ARCS OF CHORD ARC CURVES}

Our arguments are inspired by the ones used in $\left[\mathrm{ACM}^{+} 08\right]$ to obtain improved distortion results for rectifiable sets. However, we need more precise quantitative estimates. Some notation we will use is as follows. The length of an interval $I \subset \partial \mathbb{D}$ is denoted by $\ell(I)$. The side length of a square $Q$ is denoted by $\ell(Q)$. Given a 
bijective mapping $\phi: \mathbb{C} \rightarrow \mathbb{C}$ and a square $Q$, one defines the side length of $\phi(Q)$ as $\ell(\phi(Q)):=\operatorname{diam}(\phi(Q))$. Analogously, $\ell(\phi(I)):=\operatorname{diam}(\phi(I))$.

Lemma 4.1. Let $\varepsilon>0$ and let $\phi: \mathbb{C} \rightarrow \mathbb{C}$ be $a(1+\varepsilon)$-quasiconformal mapping which is conformal on $\mathbb{D}$, such that $\phi^{\prime}(0)=1$. Denote $\alpha_{0}=1-c_{0} \varepsilon^{2}$, where $c_{0}$ will be a sufficiently large constant. Let $\left\{I_{n}\right\}_{n} \subset \partial \mathbb{D}$ be a collection of pairwise disjoint dyadic intervals.

(a) If $c_{0} \geq 40$, we have

$$
\sum_{n} \ell\left(\phi\left(I_{n}\right)\right)^{\alpha_{1}} \leq C\left(\sum_{n} \ell\left(I_{n}\right)^{\alpha_{0}}\right)^{b},
$$

where $\alpha_{1}=1-\frac{1}{4} c_{0} \varepsilon^{2}$, and $b>0$ depends only on $c_{0}$; and $C$ on $c_{0}$ and $\varepsilon$.

(b) If

$$
\sum_{n} \ell\left(I_{n}\right)^{\alpha_{0}} \geq \delta
$$

then

$$
\sum_{n} \ell\left(\phi\left(I_{n}\right)\right)^{\alpha_{2}} \geq \delta^{\prime}
$$

where $\alpha_{2}=1-\left(2 c_{0}+10\right) \varepsilon^{2}$ and $\delta^{\prime}>0$ depends on $\delta, c_{0}, \varepsilon$.

Proof. (a) We drop the factor $2 \pi$ in all calculations. Let $\mathcal{D}_{j}$ be the collection of the dyadic intervals of length $2^{-j}$ of $\partial \mathbb{D}$, and set $\left\{I_{n}\right\}=\left\{I_{n}^{j}\right\}_{j, n}$, with $I_{n}^{j} \in \mathcal{D}_{j}$. Consider Whitney squares $\left\{Q_{n}^{j}\right\}_{j, n} \subset \mathbb{D}$ so that $\ell\left(Q_{n}^{j}\right) \approx \ell\left(I_{n}^{j}\right) \approx \operatorname{dist}\left(Q_{n}^{j}, I_{n}^{j}\right)$. Denote by $z_{n}^{j}$ the center of $Q_{n}^{j}$. By Koebe's distortion theorem, we have

$$
\ell\left(\phi\left(Q_{n}^{j}\right)\right) \approx\left|\phi^{\prime}\left(z_{n}^{j}\right)\right| \ell\left(Q_{n}^{j}\right) \approx\left|\phi^{\prime}\left(z_{n}^{j}\right)\right|\left(1-\left|z_{n}^{j}\right|\right) \approx\left|\phi^{\prime}(z)\right|(1-|z|),
$$

for all $z \in Q_{n}^{j}$. Denoting $\ell_{j}=\ell\left(Q_{n}^{j}\right)=2^{-j}, r_{j}=1-\ell_{j}$, and $N_{j}=\#\left\{I_{n}^{j}\right\}_{n}$, using Hölder's inequality we get

$$
\begin{aligned}
\ell_{j} \sum_{n=1}^{N_{j}} \ell\left(\phi\left(I_{n}^{j}\right)\right)^{\alpha_{1}} & \approx \ell_{j} \sum_{n=1}^{N_{j}} \ell\left(\phi\left(Q_{n}^{j}\right)\right)^{\alpha_{1}} \lesssim \int_{\bigcup_{n} I_{n}^{j}} \ell\left(I_{n}^{j}\right)^{\alpha_{1}}\left|\phi^{\prime}\left(r_{j} e^{i t}\right)\right|^{\alpha_{1}} d t \\
& =\ell_{j}^{\alpha_{1}} \int_{\bigcup_{n} I_{n}^{j}}\left|\phi^{\prime}\left(r_{j} e^{i t}\right)\right|^{\alpha_{1}} d t \\
& \leq N_{j}^{1 / p^{\prime}} \ell_{j}^{\alpha_{1}+\frac{1}{p^{\prime}}}\left[\int_{\bigcup_{n} I_{n}^{j}}\left|\phi^{\prime}\left(r_{j} e^{i t}\right)\right|^{\alpha_{1} p} d t\right]^{1 / p}
\end{aligned}
$$

for $1<p<\infty$. Since $\phi$ is $(1+\varepsilon)$-quasiconformal, we have the following estimate for the integral means:

$$
\int_{\bigcup_{n} I_{n}^{j}}\left|\phi^{\prime}\left(r_{j} e^{i t}\right)\right|^{q} d t \leq \frac{C_{\beta}}{\ell_{j}^{\beta}},
$$


with $\beta>\beta(q)$, for any $q \in \mathbb{R}$, by definition of $\beta(q)$ (see e.g Chapter 8 in [Pom92]). Recall also (e.g.[Pom92, page 182]) that, for any $q \in \mathbb{R}$,

$$
\beta(q) \leq 9\left(\frac{K-1}{K+1}\right)^{2} q^{2}
$$

So if we choose $\beta=9 \varepsilon^{2} q^{2}$, we get

$$
\sum_{n=1}^{N_{j}} \ell\left(\phi\left(I_{n}^{j}\right)\right)^{\alpha_{1}} \lesssim N_{j}^{1 / p^{\prime}} \ell_{j}^{\alpha_{1}-1+\frac{1}{p^{\prime}}-9 \varepsilon^{2} \alpha_{1}^{2} p}
$$

Replacing $N_{j}=\frac{1}{\ell_{j}^{\alpha_{0}}} \sum_{n=1}^{N_{j}} \ell\left(I_{n}^{j}\right)^{\alpha_{0}}$, we obtain

$$
\sum_{n=1}^{N_{j}} \ell\left(\phi\left(I_{n}^{j}\right)\right)^{\alpha_{1}} \lesssim\left(\sum_{n=1}^{N_{j}} \ell\left(I_{n}^{j}\right)^{\alpha_{0}}\right)^{1 / p^{\prime}} \ell_{j}^{\alpha_{1}-1+\frac{1}{p^{\prime}}-\frac{\alpha_{0}}{p^{\prime}}-9 \varepsilon^{2} \alpha_{1}^{2} p}
$$

Since $\alpha_{1} \leq 1$, if we set $\alpha_{0}=1-c_{0} \varepsilon^{2}$ and $\alpha_{1}=1-c_{1} \varepsilon^{2}$, we get

$$
\alpha_{1}-1+\frac{1}{p^{\prime}}-\frac{\alpha_{0}}{p^{\prime}}-9 \varepsilon^{2} \alpha_{1}^{2} p \geq \alpha_{1}-1+\frac{1}{p^{\prime}}-\frac{\alpha_{0}}{p^{\prime}}-9 \varepsilon^{2} p \geq \varepsilon^{2}\left(\frac{c_{0}}{p^{\prime}}-c_{1}-9 p\right)=: a .
$$

Since $c_{0} \geq 10$, we can choose $p \in(1, \infty)$ and $c_{1}>0$ such that

$$
\frac{c_{0}}{p^{\prime}}-c_{1}-9 p>0
$$

and so $a>0$ (e.g. $p=2, c_{1}=\frac{c_{0}}{4}$, and $c_{0} \geq 40$ ). By (4.2) and Hölder's inequality we get

$$
\sum_{j} \sum_{n} \ell\left(\phi\left(I_{n}^{j}\right)\right)^{\alpha_{1}} \lesssim \sum_{j}\left(\sum_{n=1}^{N_{j}} \ell\left(I_{n}^{j}\right)^{\alpha_{0}}\right)^{1 / p^{\prime}} \ell_{j}^{a} \leq\left(\sum_{j} \sum_{n=1}^{N_{j}} \ell\left(I_{n}^{j}\right)^{\alpha_{0}}\right)^{1 / p^{\prime}}\left(\sum_{j} \ell_{j}^{a p}\right)^{1 / p} .
$$

Since $a>0$, we have $\sum_{j} \ell_{j}^{a p} \leq C\left(c_{0}, \varepsilon\right)$, and the statement (a) in the lemma follows.

(b) We use the same notation as in (a). Let $\ell_{\max }=\max _{n} \ell\left(I_{n}\right)$, and denote

$$
Z_{j}=\left\{I_{n}^{j}:\left|\phi^{\prime}\left(z_{n}^{j}\right)\right| \leq \ell\left(I_{n}^{j}\right)^{\gamma}\right\}
$$

where $\gamma>0$ is some small constant to be chosen below. Then we have

$$
\ell_{j}^{1-\gamma} \# Z_{j} \lesssim \int_{\mathbf{T}} \frac{d t}{\left|\phi^{\prime}\left(r_{j} e^{i t}\right)\right|} \leq \frac{C(\beta)}{\ell_{j}^{\beta}}
$$

for $\beta>\beta(-1)$. So we infer that

$$
\sum_{I \in Z_{j}} \ell(I)^{\alpha_{0}}=\ell_{j}^{\alpha_{0}} \# Z_{j} \leq C(\beta) \ell_{j}^{\gamma-\beta+\alpha_{0}-1}
$$

Assuming that

$$
\gamma-\beta+\alpha_{0}-1>0
$$


summing on $j \geq 0$ and setting $Z=\bigcup_{j} Z_{j}$, we get

$$
\sum_{I \in Z} \ell(I)^{\alpha_{0}} \leq C(\beta) \sum_{j \geq 0} \ell_{j}^{\gamma-\beta+\alpha_{0}-1} \leq C(\beta, \gamma, \varepsilon) \ell_{\max }^{\gamma-\beta+\alpha_{0}-1} .
$$

Therefore, if $\ell_{\max }$ is small enough (depending on $\left.\beta, \gamma, \delta, \varepsilon\right)$ we infer that $\sum_{I \in Z} \ell(I)^{\alpha_{0}} \leq$ $\frac{\delta}{2}$, and so

$$
\sum_{I \notin Z} \ell(I)^{\alpha_{0}} \geq \frac{\delta}{2}
$$

For the intervals $I \notin Z$ we use (4.1), and we obtain

$$
\ell(I) \approx \frac{1}{\left|\phi^{\prime}\left(z_{I}\right)\right|} \ell(\phi(I)) \leq \frac{\ell(\phi(I))}{\ell(I)^{\gamma}}
$$

where $z_{I}=z_{n}^{j}$ if $I=I_{n}^{j}$. We deduce

$$
\frac{\delta}{2} \leq \sum_{I \notin Z} \ell(I)^{\alpha_{0}} \leq C \sum_{I \notin Z} \ell(\phi(I))^{\alpha_{0} /(1+\gamma)} .
$$

Therefore, (b) holds if $\ell_{\max }$ is small enough and we choose $\beta$ and $\gamma$ such that (4.3) is true, that is, if

$$
\gamma>\beta+c_{0} \varepsilon^{2}
$$

Using the estimate (see e.g. [Pom92, page 182])

$$
\beta(-q) \leq 9\left(\frac{K-1}{K+1}\right)^{2} q^{2}
$$

we derive $\beta(-1) \leq 9 \varepsilon^{2}$. Thus, (4.5) holds if we choose

$$
\gamma=\left(10+c_{0}\right) \varepsilon^{2}
$$

say. Then we have

$$
\frac{\alpha_{0}}{1+\gamma}=\frac{1-c_{0} \varepsilon^{2}}{1+\left(10+c_{0}\right) \varepsilon^{2}} \geq\left(1-c_{0} \varepsilon^{2}\right)\left(1-\left(10+c_{0}\right) \varepsilon^{2}\right) \geq 1-\left(10+2 c_{0}\right) \varepsilon^{2}
$$

From (4.4) we deduce

$$
C \delta \leq \sum_{n} \ell\left(\phi\left(I_{n}\right)\right)^{1-\left(10+2 c_{0}\right) \varepsilon^{2}}
$$

if $\ell_{\max }$ is small enough, i.e. if $\ell_{\max } \leq l_{0}$, where $l_{0}$ is some constant depending on $c_{0}, \varepsilon, \delta$.

The case where $\ell_{\max }$ is not small follows easily from the preceding estimates. Indeed, let $\mathcal{F}$ be the family of dyadic intervals obtained by splitting each interval $I_{n}$ into $2^{N}$ pairwise disjoint dyadic intervals, with $N$ big enough so that each interval from $\mathcal{F}$ has length smaller than $l_{0}$. If we have

$$
I_{n}=I_{1}^{\prime} \cup \ldots \cup I_{2^{N}}^{\prime}
$$


with $I_{j}^{\prime} \in \mathcal{F}$, then we get

$$
\ell\left(I_{n}\right)^{\alpha_{0}} \leq \sum_{j=1}^{2^{N}} \ell\left(I_{j}^{\prime}\right)^{\alpha_{0}},
$$

and thus, $\delta \leq \sum_{I \in \mathcal{F}} \ell(I)^{\alpha_{0}}$. So we infer that

$$
C \delta \leq \sum_{I \in \mathcal{F}} \ell(\phi(I))^{1-\left(10+2 c_{0}\right) \varepsilon^{2}} \leq 2^{N} \sum_{n} \ell\left(\phi\left(I_{n}\right)\right)^{1-\left(10+2 c_{0}\right) \varepsilon^{2}},
$$

with $N$ depending on $c_{0}, \varepsilon, \delta$.

Lemma 4.2. Let $\varepsilon>0$ and let $\phi: \mathbb{C} \rightarrow \mathbb{C}$ be a $(1+\varepsilon)$-quasiconformal mapping. Denote $\alpha_{0}=1-c_{0} \varepsilon^{2}$. Let $\left\{I_{n}\right\}_{n} \subset \partial \mathbb{D}$ be a collection of pairwise disjoint dyadic intervals such that

$$
\sum_{n} \ell\left(I_{n}\right)^{\alpha_{0}} \geq \delta_{0}
$$

Then we have

$$
\sum_{n} \ell\left(\phi\left(I_{n}\right)\right)^{\alpha} \geq \delta \operatorname{diam}(\phi(\mathbb{D}))^{\alpha},
$$

where $\alpha=1-C \varepsilon^{2}$ (and $C$ depends on $c_{0}$ ), and $\delta>0$, which depends on $\delta_{0}, c_{0}, \varepsilon$ (i.e. $\delta=\delta\left(\delta_{0}, c_{0}, \varepsilon\right)$ ).

Proof. The lemma follows by combining (a) and (b) in the preceding lemma: arguing as in [ACM $\left.{ }^{+} 08\right]$, we write $\phi=f \circ g^{-1} \circ h$, so that $f, g, h$ are $(1+C \varepsilon)$-quasiconformal and moreover $h$ is principal and conformal on $\mathbb{C} \backslash \mathbb{D}$ (and so $\operatorname{diam}(h(\mathbb{D})) \approx 1), f, g$ are conformal on $\mathbb{D}$, and $f(\mathbb{D})=\phi(\mathbb{D})$ and $g(\mathbb{D})=h(\mathbb{D})$. So

$$
\mathbb{D} \stackrel{h}{\longrightarrow} h(\mathbb{D}) \stackrel{g^{-1}}{\longrightarrow} \mathbb{D} \stackrel{f}{\longrightarrow} \phi(\mathbb{D}) .
$$

From (b) in Lemma 4.1 we infer that

$$
\sum_{n} \ell\left(h\left(I_{n}\right)\right)^{\alpha^{\prime}} \geq \delta^{\prime}
$$

with $\alpha^{\prime}=1-C^{\prime} \varepsilon^{2}$. Here $C^{\prime}=C^{\prime}\left(c_{0}\right)$, and $\delta^{\prime}=\delta^{\prime}\left(\delta_{0}, c_{0}, \varepsilon\right)$. By (a) in the same lemma we get

$$
\sum_{n} \ell\left(g^{-1} \circ h\left(I_{n}\right)\right)^{\alpha^{\prime \prime}} \geq \delta^{\prime \prime},
$$

with $\alpha^{\prime \prime}=1-C^{\prime \prime} \varepsilon^{2}$. Here $C^{\prime \prime}=C^{\prime \prime}\left(C^{\prime}\right)$, and $\delta^{\prime \prime}=\delta^{\prime \prime}\left(\delta^{\prime}, C^{\prime}, \varepsilon\right)$. And by (b) again,

$$
\sum_{n} \ell\left(f \circ g^{-1} \circ h\left(I_{n}\right)\right)^{\alpha^{\prime \prime \prime}} \geq \delta^{\prime \prime \prime} \operatorname{diam}(\phi(\mathbb{D}))^{\alpha^{\prime \prime \prime}},
$$

where $\alpha^{\prime \prime \prime}=1-C^{\prime \prime \prime} \varepsilon^{2}$. Here $C^{\prime \prime \prime}=C^{\prime \prime \prime}\left(C^{\prime \prime}\right)$, and $\delta^{\prime \prime \prime}=\delta^{\prime \prime \prime}\left(\delta^{\prime \prime}, C^{\prime \prime}, \varepsilon\right)$. 
Lemma 4.3. Let $\phi: \mathbb{C} \rightarrow \mathbb{C}$ be a $K$-quasiconformal mapping. Let $\left\{I_{n}\right\}_{n}$ be a family of pairwise disjoint sub-arcs of $\partial \mathbb{D}$ such that

$$
\sum_{n} \ell\left(I_{n}\right) \geq \delta
$$

with $\delta>0$. Then,

$$
\sum_{n} \ell\left(\phi\left(I_{n}\right)\right)^{\alpha} \geq \delta^{\prime} \operatorname{diam}(\phi(\mathbb{D}))^{\alpha}
$$

where $\delta^{\prime}$ is a positive constant depending only on $K, \delta$; and $\alpha$ depends only on $K$ and verifies

$$
\frac{2}{K+1}<\alpha<1
$$

Proof. By appropriate standard arguments, we may assume that $\operatorname{diam}(\phi(\mathbb{D}))=1$. We factorize $\phi=\phi_{2} \circ \phi_{1}$ so that $\phi_{i}, i=1,2$ are $K_{i}$-quasiconformal, with $K_{1}=1+\varepsilon$ and $K_{2}=K / K_{1}$, and so that $\operatorname{diam}\left(\phi_{1}(\mathbb{D})\right)=1$. By quasi-symmetry we may assume that the intervals $I_{n}$ are dyadic. (See e.g. [AIM09, Chapter 3] for the definition of quasisymmetry and its equivalence with quasiconformality. A consequence of this definition is that both $\delta$ and $\delta^{\prime}$ in the statement only change by a constant factor which only depends on $K$ if each of the intervals $I_{n}$ is replaced by a "nearby" dyadic interval which has comparable size to $I_{n}$.) By Lemma 4.2 we have

$$
\sum_{n} \ell\left(\phi_{1}\left(I_{n}\right)\right)^{\alpha_{1}} \geq \delta_{1}
$$

with

$$
\eta:=\alpha_{1}-\frac{2}{K_{1}+1}>0
$$

if $\varepsilon$ is small enough.

To estimate the distortion of the $\operatorname{arcs} \phi_{1}\left(I_{n}\right)$, we consider a family of pairwise disjoint balls $B_{n}$ centered on $\phi_{1}\left(I_{n}\right)$ with radii $r_{n} \approx \ell\left(\phi_{1}\left(I_{n}\right)\right)$, and so that $\operatorname{diam}\left(\phi_{2}\left(B_{n}\right)\right) \approx$ $\ell\left(\phi\left(I_{n}\right)\right)$. Take a constant $K_{2}^{\prime}>K_{2}$ to be fixed below. By Hölder's inequality, we have

$$
\begin{aligned}
\sum_{n} \ell\left(\phi_{1}\left(I_{n}\right)\right)^{\alpha_{1}} & \approx \sum_{n} r_{n}^{\alpha_{1}} \lesssim \sum_{n}\left(\int_{\phi_{2}\left(B_{n}\right)} J\left(\phi_{2}^{-1}\right) d x\right)^{\alpha_{1} / 2} \\
& \leq \sum_{n}\left(\int_{\phi_{2}\left(B_{n}\right)} J\left(\phi_{2}^{-1}\right)^{\frac{K_{2}^{\prime}}{K_{2}^{\prime}-1}} d x\right)^{\frac{\alpha_{1}\left(K_{2}^{\prime}-1\right)}{2 K_{2}^{\prime}}} \ell\left(\phi\left(I_{n}\right)\right)^{\frac{\alpha_{1}}{K_{2}^{\prime}}} \\
& \leq\left(\sum_{n} \int_{\phi_{2}\left(B_{n}\right)} J\left(\phi_{2}^{-1}\right)^{\frac{K_{2}^{\prime}}{K_{2}^{\prime}-1}} d x\right)^{1 / p}\left(\sum_{n} \ell\left(\phi\left(I_{n}\right)\right)^{\frac{\alpha_{1} p^{\prime}}{K_{2}^{\prime}}}\right)^{1 / p^{\prime}}
\end{aligned}
$$

where we chose

$$
\frac{1}{p}=\frac{\alpha_{1}\left(K_{2}^{\prime}-1\right)}{2 K_{2}^{\prime}}
$$


Notice that $K_{2}^{\prime} /\left(K_{2}^{\prime}-1\right)<K_{2} /\left(K_{2}-1\right)$ and then

$$
\sum_{n} \int_{\phi_{2}\left(B_{n}\right)} J\left(\phi_{2}^{-1}\right)^{\frac{K_{2}^{\prime}}{K_{2}^{\prime}-1}} d x \leq \int J\left(\phi_{2}^{-1}\right)^{\frac{K_{2}^{\prime}}{K_{2}^{\prime}-1}} d x \leq C\left(K_{2}, K_{2}^{\prime}\right)<\infty .
$$

The last estimate follows from our normalizations $\left(\operatorname{diam}\left(\phi_{1}(\mathbb{D})\right)=\operatorname{diam}(\phi(\mathbb{D}))=1\right)$ and the higher integrability of quasiconformal maps (see e.g. equation (13.24) in AIM09]), which is in turn a consequence of the celebrated area distortion theorem proved by K. Astala in Ast94. So we get

$$
\delta_{1} \leq \sum_{n} \ell\left(\phi_{1}\left(I_{n}\right)\right)^{\alpha_{1}} \leq C\left(\sum_{n} \ell\left(\phi\left(I_{n}\right)\right)^{\frac{\alpha_{1} p^{\prime}}{K_{2}^{\prime}}}\right)^{1 / p^{\prime}} .
$$

To show that the lemma holds in this particular case, it is enough to take

$$
\alpha:=\frac{\alpha_{1} p^{\prime}}{K_{2}^{\prime}}
$$

and then it remains to check that $2 /(K+1)<\alpha<1$. To this end, observe that, by (4.6) and (4.7),

$$
\frac{1}{p}>\frac{K_{2}^{\prime}-1}{\left(K_{1}+1\right) K_{2}^{\prime}}
$$

and thus

$$
\frac{1}{p^{\prime}}<\frac{K_{1} K_{2}^{\prime}+1}{\left(K_{1}+1\right) K_{2}^{\prime}}
$$

From this estimate and (4.6) we obtain

$$
\frac{\alpha_{1} p^{\prime}}{K_{2}^{\prime}}>\left(\frac{2}{K_{1}+1}+\eta\right) \frac{K_{1}+1}{K_{1} K_{2}^{\prime}+1}=\frac{2}{K_{1} K_{2}^{\prime}+1}+\eta \frac{K_{1}+1}{K_{1} K_{2}^{\prime}+1}
$$

From this inequality (with given $K=K_{1} K_{2}$ and $\eta$ ) it is clear that if $K_{2}^{\prime}$ is close enough to $K_{2}$ (with $K_{2}^{\prime}>K_{2}$ ), then

$$
\alpha=\frac{\alpha_{1} p^{\prime}}{K_{2}^{\prime}}>\frac{2}{K+1}
$$

To show that $\alpha<1$, notice that (4.7) implies that

$$
\frac{1}{p}<\frac{K_{2}^{\prime}-1}{2 K_{2}^{\prime}}
$$

and then one easily gets

$$
p^{\prime}<\frac{2 K_{2}^{\prime}}{K_{2}^{\prime}+1}
$$

and thus

$$
\alpha=\frac{\alpha_{1} p^{\prime}}{K_{2}^{\prime}}<\frac{p^{\prime}}{K_{2}^{\prime}}<\frac{2}{K_{2}^{\prime}+1}<1
$$

since $K_{2}^{\prime}>K_{2} \geq 1$. 
Remark 4.4. The preceding arguments show that, choosing a suitable $K_{2}^{\prime}$, one gets

$$
\alpha \geq \frac{2}{K+1}+\frac{\eta}{2} \frac{K_{1}+1}{K+1} \geq \frac{2}{K+1}+\frac{\eta}{2} \frac{1}{K+1} .
$$

Lemma 4.5. Let $\phi: \mathbb{C} \rightarrow \mathbb{C}$ be a principal $K$-quasiconformal mapping, and let $\Gamma \subset \mathbb{C}$ be a chord arc curve. Let $\left\{I_{n}\right\}_{n}$ be a family of pairwise disjoint subarcs of $\Gamma$ such that

$$
\sum_{n} \ell\left(I_{n}\right) \geq \delta \operatorname{diam}(\Gamma)
$$

with $\delta>0$. If the chord arc constant $C_{\Gamma}$ is close enough to 1 , that is, $\left|C_{\Gamma}-1\right| \leq \varepsilon_{0}$ with $\varepsilon_{0}=\varepsilon_{0}(K)$, then

$$
\sum_{n} \ell\left(\phi\left(I_{n}\right)\right)^{\alpha} \geq \delta^{\prime} \operatorname{diam}(\phi(\Gamma))^{\alpha},
$$

where $\delta^{\prime}$ is a positive constant depending only on $K, \delta$, and the chord arc constant; and $\alpha$ depends only on $K$ and verifies

$$
\frac{2}{K+1}<\alpha
$$

Recall that a chord arc curve is the bilipschitz image of an interval. The chord arc constant is the bilipschitz constant (or rather the infimum over all the possible bilipschitz constants).

Notice that the above result can be understood as a quantitative version of the result of $\left[\mathrm{ACM}^{+} 08\right]$ which asserts that if $F$ is rectifiable and of positive length, then $\operatorname{dim}(\phi(F))>2 /(K+1)+c(K)$, where $c(K)$ is some positive constant depending only on $K$.

Proof of Lemma 4.5. If $\Gamma$ is an arc of a circumference or a segment, then the result follows from Lemma 4.3 by appropriate normalization.

In the case of a general chord arc curve with small constant, we consider a bilipschitz parametrization $f: J \rightarrow \Gamma$, where $J$ is a segment with $\ell(J)=\operatorname{diam}(\Gamma)$, so that the bilipschitz constant $C_{f}$ of $f$ is very close to 1 :

$$
\left|C_{f}-1\right| \leq c(K) \quad \text { with } c(K) \rightarrow 0 \text { as } \varepsilon_{0}(K) \rightarrow 0 .
$$

By a theorem of Väisälä Väi86], $f$ can be extended to a bilipschitz mapping $\tilde{f}$ : $\mathbb{C} \rightarrow \mathbb{C}$ with constant $C_{\widetilde{f}}$ depending on $C_{f}$ very close to 1 too. In particular $\tilde{f}$ is quasiconformal with constant $K_{\tilde{f}} \rightarrow 1$ as $\varepsilon_{0}(K) \rightarrow 0$.

Using the auxiliary mapping $\phi_{0}=\phi \circ f$, we deduce from Lemma 4.3 that

$$
\sum_{n} \ell\left(\phi\left(I_{n}\right)\right)^{\alpha} \geq \delta^{\prime} \operatorname{diam}(\Gamma)^{\alpha}
$$

with $\alpha$ such that $\alpha>\frac{2}{K K_{\widetilde{f}}+1}$. By Remark 4.4, for $K_{\widetilde{f}}$ close enough to 1 , we have

$$
\alpha>\frac{2}{K+1}
$$




\section{A COROna type Decomposition For measures With Finite CURVATURe AND LINEAR GROWTH}

Throughout all this section we suppose that $\mu$ is supported on $E \subset B(0,1 / 2)$, and satisfies

$$
\mu(B(x, r)) \leq r \quad \text { for all } x \in \mathbb{C}, r>0 ; \quad c_{\mu}^{2}(x) \leq 1 \quad \text { for all } x \in \mathbb{C} .
$$

As explained in Section [3, our objective is to construct a corona type decomposition for $\mu$, which has some similarities with the one of [Tol05]. This corona type decomposition will be used in Section 6 to find a measure $\nu$ supported on $\phi(E)$ with bounded potential $\dot{W}_{\frac{2 K}{2 K+1}}^{\nu}, \frac{2 K+1}{K+1}$.

5.1. Additional notation and terminology. By a square we mean a square with sides parallel to the axes. Moreover, we assume the squares to be half closed half open. The side length of a square $Q$ is denoted by $\ell(Q)$. If $\ell(Q)=2^{-n}$, then we write $J(Q)=n$. Given $a>0, a Q$ denotes the square concentric with $Q$ with side length $a \ell(Q)$. A square $Q \subset \mathbb{C}$ is called 4-dyadic if it is of the form $\left[j 2^{-n},(j+4) 2^{-n}\right) \times\left[k 2^{-n},(k+4) 2^{-n}\right)$, with $j, k, n \in \mathbb{Z}$. So a 4-dyadic square with side length $4 \cdot 2^{-n}$ is made up of 16 dyadic squares with side length $2^{-n}$.

Given $a, b>1$, the square $Q$ is $(a, b)$-doubling if $\mu(a Q) \leq b \mu(Q)$. If we don't want to specify the constant $b$, we say that $Q$ is $a$-doubling. If $h_{a}$ is the function defined in (2.4), we say that $Q$ is $\left(h_{a}, b\right)$-doubling if

$$
h_{a}(Q) \leq b \mu(Q),
$$

which is equivalent to $\varepsilon_{a}(Q) \leq b \theta_{\mu}(Q)$. Notice that if $Q$ is $\left(h_{a}, b\right)$-doubling, then, for all $c>1$ there exists some $d>0$ depending only on $a, b, c$ such that $Q$ is $(c, d)$-doubling.

Given a bijective mapping $\phi: \mathbb{C} \rightarrow \mathbb{C}$ and a square $Q$, one says that that $\phi(Q)$ is a $\phi$-square, and then one defines its side length as $\ell(\phi(Q)):=\operatorname{diam}(Q)$. If $Q_{0}$ is a dyadic (or 4-dyadic) square, we say that $\phi\left(Q_{0}\right)$ is a dyadic (or 4-dyadic) $\phi$-square. If $Q=\phi\left(Q_{0}\right)$ is a $\phi$-square, we denote $\lambda Q=\phi\left(\lambda Q_{0}\right)$, for $\lambda>0$.

An Ahlfors regular curve is a curve $\Gamma$ such that $\mathcal{H}^{1}(\Gamma \cap B(x, r)) \leq C r$ for all $x \in \Gamma, r>0$, and some fixed $C>0$. Recall that $\Gamma$ is a chord arc curve if it is a bilipschitz image of an interval in $\mathbb{R}$. If the bilipschitz constant of the map is $L$, we say that $\Gamma$ is an $L$-chord arc curve.

The total Menger curvature of $\mu$ is

$$
c^{2}(\mu)=\int c_{\mu}^{2}(x) d \mu(x)
$$



with $c_{\mu}^{2}(x)$ defined by (1.3) . The curvature operator $K_{\mu}$ is

$$
K_{\mu}(f)(x)=\int k_{\mu}(x, y) f(y) d \mu(y), \quad f \in L_{l o c}^{1}(\mu), x \in \mathbb{C},
$$

where $k_{\mu}(x, y)$ is the kernel

$$
k_{\mu}(x, y)=\int \frac{1}{R(x, y, z)^{2}} d \mu(z), \quad x, y \in \mathbb{C} .
$$

For $j \in \mathbb{Z}$, the truncated operators $K_{\mu, j}, j \in \mathbb{Z}$, are defined as

$$
K_{\mu, j} f(x)=\int_{|x-y|>2^{-j}} k_{\mu}(x, y) f(y) d \mu(y), \quad f \in L_{l o c}^{1}(\mu), x \in \mathbb{C} .
$$

Notice that $c_{\mu}^{2}(x)=K_{\mu}\left(\chi_{E}\right)(x)$.

\subsection{Properties of $\left(h_{a}, b\right)$-doubling squares.}

Remark 5.1. Let $Q$ be a square and $x$ its center. For $N \geq 1$, we have

$$
\begin{aligned}
\varepsilon_{a}(Q) & \approx \frac{1}{\ell(Q)} \int \frac{1}{\left(\frac{|x-y|}{\ell(Q)}\right)^{1+a}+1} d \mu(y) \\
& \leq C \sum_{j=0}^{N} \frac{1}{\ell(Q)} \frac{\mu\left(2^{j} Q\right)}{2^{j(1+a)}}+\frac{1}{\ell(Q)} \int_{\mathbb{C} \backslash Q_{N}} \frac{1}{\left(\frac{|x-y|}{\ell(Q)}\right)^{1+a}} d \mu(y),
\end{aligned}
$$

where $Q_{N}:=2^{N} Q$ and the constant $C$ depends on $a$ but on $N$. Since

$\frac{1}{\ell(Q)} \int_{\mathbb{C} \backslash Q_{N}} \frac{1}{\left(\frac{|x-y|}{\ell(Q)}\right)^{1+a}} d \mu(y)=\frac{2^{-a N}}{\ell\left(Q_{N}\right)} \int_{\mathbb{C} \backslash Q_{N}} \frac{1}{\left(\frac{|x-y|}{\ell\left(Q_{N}\right)}\right)^{1+a}} d \mu(y) \leq C(a) 2^{-a N} \varepsilon_{a}\left(Q_{N}\right)$,

we deduce

$$
\varepsilon_{a}(Q) \leq C(a)\left(\sum_{j=0}^{N-1} 2^{-a j} \theta_{\mu}\left(2^{j} Q\right)+2^{-a N} \varepsilon_{a}\left(Q_{N}\right)\right) .
$$

The converse inequality is also true, but we will not need it.

Lemma 5.2. Given $a>0$, let $b>0$ be some big enough constant. Let $Q$ be a square, and suppose that $2^{-j} Q$ is not $\left(h_{a}, b\right)$-doubling for $0 \leq j \leq N$. Then,

$$
\theta_{\mu}\left(2^{-j} Q\right) \leq 2^{-a j / 2} \varepsilon_{a}(Q) \quad \text { for } 0 \leq j \leq N,
$$

and

$$
\sum_{j=0}^{N} \varepsilon_{a}\left(2^{-j} Q\right)^{2} \leq C \varepsilon_{a}(Q)^{2}
$$

with $C$ independent of $N$. 
Proof. By (5.1), the fact that $2^{-j} Q$ is not $\left(h_{a}, b\right)$-doubling for $0 \leq j \leq N$ implies that

$$
\theta_{\mu}\left(2^{-j} Q\right) \leq \frac{1}{b} \varepsilon_{a}\left(2^{-j} Q\right) \leq \frac{C_{3}}{b}\left(\sum_{k=1}^{j-1} 2^{-a k} \theta_{\mu}\left(2^{-j+k} Q\right)+2^{-a j} \varepsilon_{a}(Q)\right)
$$

where $C_{3}$ depends on $a$. Notice that the sum above starts with $k=1$, while the one in (5.1) starts with $j=0$ (we used the fact that $\theta_{\mu}\left(2^{-j} Q\right) \leq C \theta_{\mu}\left(2^{-j+1} Q\right)$ ).

We prove (5.2) by induction on $j$. For $j=0$, this is a direct consequence of the definition of $\left(h_{a}, b\right)$-doubling squares. Suppose that (5.2) holds for $0 \leq h \leq j$, with $j \leq N-1$, and consider the case $j+1$. Using (5.4) and the induction hypothesis we get

$$
\begin{aligned}
\theta_{\mu}\left(2^{-j-1} Q\right) & \leq \frac{C_{3}}{b}\left(\sum_{k=1}^{j} 2^{-a k} \theta_{\mu}\left(2^{-j-1+k} Q\right)+2^{-a(j+1)} \varepsilon_{a}(Q)\right) \\
& \leq \frac{C_{3}}{b}\left(\sum_{k=1}^{j} 2^{-a k} 2^{(-j-1+k) a / 2} \varepsilon_{a}(Q)+2^{-a(j+1)} \varepsilon_{a}(Q)\right)
\end{aligned}
$$

Since

$$
\sum_{k=1}^{j} 2^{-a k} 2^{(-j-1+k) a / 2} \leq C(a) 2^{-a j / 2}
$$

we obtain

$$
\theta_{\mu}\left(2^{-j-1} Q\right) \leq \frac{C_{3} C(a)}{b}\left(2^{-a j / 2}+2^{-a(j+1)}\right) \varepsilon_{a}(Q) .
$$

If $b$ is chosen big enough, we get

$$
\theta_{\mu}\left(2^{-j-1} Q\right) \leq 2^{-a(j+1) / 2} \varepsilon_{a}(Q)
$$

The estimate (5.3) is a straightforward consequence of (5.2), using Cauchy-Schwartz inequality. We leave the details for the reader.

Let $b=b(a)>0$ be big enough so that (5.2) and (5.3) hold. It is immediate to check that if $Q$ is $\left(h_{a}, b\right)$-doubling and $R \supset Q$ is a square such that $\ell(R) \leq 4 \ell(Q)$, then $R$ is $\left(h_{a}, C_{4} b\right)$-doubling. We say that a square $R$ is $h_{a}$-doubling if it is $\left(h_{a}, C_{4} b\right)$ doubling.

Let $Q, R$ be squares with $\ell(Q) \leq \ell(R)$. We denote

$$
D_{\mu}(Q, R)=\sum_{j: Q \subset 2^{j} Q \subset R_{Q}} \varepsilon_{a}\left(2^{j} Q\right)^{2},
$$

where $R_{Q}$ denoted the smallest square of the form $2^{j} Q$ that contains $R$. The preceding lemma says that if $Q \subset R$ and there are no $h_{a}$-doubling squares of the form $2^{j} Q$ such that $Q \subset 2^{j} Q \subset R_{Q}$, then $D_{\mu}(Q, R) \leq C \varepsilon_{a}(R)^{2}$.

The definition of $D_{\mu}(Q, R)$ can be extended in a natural way to the case where $Q$ is replaced by a point. In this case the sum above runs over all squares centered 
at $x$ with side length $2^{j}, j \in \mathbf{Z}$, which are contained in $R_{x}$, where $R_{x}$ is the smallest square centered at $x$ that contains $R$.

Remark 5.3. Let $\mu$ be any Radon measure on $\mathbb{C}$, and let $d$ be big enough. Then, for $\mu$-almost all $x \in E$, there exists a sequence of $(2, d)$-doubling squares $\left\{Q_{n}\right\}_{n}$ centered at $x$ such that $\ell\left(Q_{n}\right) \rightarrow 0$. However, this statement is false if we replace $(2, d)$-doubling squares by $\left(h_{a}, d\right)$-doubling squares when $a$ is small. The reader can check that this is the case for planar Lebesgue measure, for instance.

5.3. The family $\operatorname{Bad}(R)$. Let $R$ be some fixed 4-dyadic square such that $\frac{1}{2} R$ is $h_{a^{-}}$ doubling. In this subsection we will explain the construction of a family of 4-dyadic squares called $\operatorname{Bad}(R)$.

Let $A>10$ be some big constant to be chosen below, $\delta$ some small positive constant $\left(\delta<1 / 10\right.$, say) which depends on $A$; and $\varepsilon_{0}$ another small constant with $0<\varepsilon_{0}<1 / 100$ (depending on $A$ and $\delta$ ). Let $Q$ be a square centered at some point in $3 R \cap \operatorname{supp}(\mu)$, with $\ell(Q)=2^{-n} \ell(R), n \geq 5$. We introduce the following notation:

(a) If $\theta_{\mu}(Q) \geq A \theta_{\mu}(R)$, then we write $Q \in H D_{c}(R)$ (high density).

(b) If $Q \notin H D_{c}(R)$ and

$$
\mu\left\{x \in Q: K_{\mu, J(Q)+10} \chi_{E}(x)-K_{\mu, J(R)-2} \chi_{E}(x) \geq \varepsilon_{0} \theta_{\mu}(R)^{2}\right\} \geq \frac{1}{2} \mu(Q),
$$

then we set $Q \in H C_{c}(R)$ (high curvature).

(c) If $Q \notin H D_{c}(R) \cup H C_{c}(R)$ and there exists some square $S_{Q}$ such that $Q \subset$ $\frac{1}{100} S_{Q}$, with $\ell\left(S_{Q}\right) \leq \ell(R) / 8$ and $\theta_{\mu}\left(S_{Q}\right) \leq \delta \theta_{\mu}(R)$, then we set $Q \in L D_{c}(R)$ (low density).

For each point $x \in 3 R \cap \operatorname{supp}(\mu)$ which belongs to some square from $H D_{c}(R) \cup$ $H C_{c}(R) \cup L D_{c}(R)$ consider the largest square $Q_{x} \in H D_{c}(R) \cup H C_{c}(R) \cup L D_{c}(R)$ which contains $x$. Let $\widehat{Q}_{x}$ be a 4-dyadic square with side length $4 \ell\left(Q_{x}\right)$ such that $Q_{x} \subset \frac{1}{2} \widehat{Q}_{x}$. Now we apply Besicovitch's covering theorem to the family $\left\{\widehat{Q}_{x}\right\}_{x}$ (notice that this theorem can be applied because $x \in \frac{1}{2} \widehat{Q}_{x}$ ), and we obtain a family of 4-dyadic squares $\left\{\widehat{Q}_{x_{i}}\right\}_{i}$ with finite overlap such that the union of the squares from $H D_{c}(R) \cup H C_{c}(R) \cup L D_{c}(R)$ is contained (as a set in $\mathbb{C}$ ) in $\bigcup_{i} \widehat{Q}_{x_{i}}$. We define

$$
\operatorname{Bad}(R):=\left\{\widehat{Q}_{x_{i}}\right\}_{i} .
$$

Notice that the squares $Q \in \operatorname{Bad}(R)$ satisfy $\ell(Q) \leq \ell(R) / 8$. If $Q_{x_{i}} \in H D_{c}(R)$, then we write $\widehat{Q}_{x_{i}} \in H D(R)$, and analogously with $H C_{c}(R), L D_{c}(R)$ and $H C(R)$, $L D(R)$. We also denote

$$
G(R)=3 R \backslash \bigcup_{Q \in \operatorname{Bad}(R)} Q .
$$

Remark 5.4. The constants that we denote by $C$ (with or without subindex) in the rest of Section 5 do not depend on $A, \delta$, or $\varepsilon_{0}$, unless stated otherwise. 
To define the squares $\operatorname{Bad}(R)$ we have followed quite closely the arguments in Tol05]. However, there are a couple of small changes: in [Tol05] we ask the square $R$ to be $(70,5000)$-doubling instead of $\left(h_{a}, b\right)$-doubling. Moreover, in [Tol05] the squares from $H D_{c}(R), L D_{c}(R)$, and $H C_{c}(R)$ are asked to be $(70,5000)$-doubling and then the resulting squares from $\operatorname{Bad}(R)$ are $(16,5000)$-doubling. Now, for convenience, we have not asked any doubling condition on these squares, although below we will need other stopping squares to be doubling (in fact, $h_{a}$-doubling).

The squares from $\operatorname{Bad}(R)$ satisfy the following important properties:

Lemma 5.5. Let $0<\rho<1$ be some fixed constant. Let $R$ be a 4-dyadic square such that $\frac{1}{2} R$ is $h_{a}$-doubling. Given $A$ and $\delta$ as above, if $\varepsilon_{0}$ is chosen small enough (depending on $A, \delta, \rho)$, there are constants $C_{5}=C_{5}(A, \delta)>1$ and $C_{6}=C_{6}(A, \delta)>0$, and there are $N_{0}$ chord arc curves with constant $(1+\rho)$ whose union we denote by $\Gamma_{R}$ with the following properties:

(a) $G(R) \subset \Gamma_{R}$;

(b) any square $Q \in \operatorname{Bad}(R)$ satisfies

$$
C_{5} Q \cap \Gamma_{R} \neq \varnothing
$$

(c) if $P$ is a square concentric with $Q \in \operatorname{Bad}(R)$ and $C_{5} \ell(Q) \leq \ell(P) \leq \ell(R)$, then

$$
C_{6}^{-1} \theta_{\mu}(R) \leq \theta_{\mu}(P) \leq C_{6} \theta_{\mu}(R) .
$$

The constant $N_{0}$ depends only on $A, \delta$, and $\rho$.

For the proof of this lemma, see [Tol05, Section 4]. One only needs to make very minor adjustments for that arguments to work in our situation. See also [CT08, Subsection 2.3] concerning the fact that one can take chord arc curves (in the original arguments in [Tol05] $\Gamma_{R}$ turns out to be an AD regular curve). We will not go through the details.

Remark 5.6. It is easy to check that the property (c) in the preceding lemma implies that the squares $P$ from $(c)$ are $\left(h_{a}, c\right)$-doubling, with $c$ depending on $A$ and $\delta$.

We also have:

Lemma 5.7. Given $A>0$, if $\delta$ and $\varepsilon_{0}$ are chosen small enough, then for any 4-dyadic square $R$ with $\frac{1}{2} R h_{a}$-doubling, we have

$$
\mu\left(\bigcup_{Q \in L D(R)} Q\right) \leq \frac{1}{100} \mu(R) .
$$

For the proof, see [Tol05, Section 7]. Again, the arguments there work with very minor adjustments. 
5.4. The families $\operatorname{Sel}(\mu), \operatorname{Sel}_{S}(\mu)$, and $\operatorname{Sel}_{L}(\mu)$. In the corona construction from Tol05] one constructs recursively the familiy of squares $\operatorname{Top}(\mu)$ mentioned in Section 3. In this subsection we construct a quite analogous family which we will denote by $\operatorname{Sel}(\mu)$ (the "selected squares"). We use another notation because the family $\operatorname{Sel}(\mu)$ will have significant differences with respect the family $\operatorname{Top}(\mu)$ of [Tol05].

First we have to distinguish two types of $h_{a}$-doubling squares:

Definition 5.8. Let $\eta>0$ be some constant to be fixed below (in Section 7 ), which will depend on $A, \delta, \varepsilon_{0}, \rho, K$ (recall that $K$ is the distortion of the quasiconformal mapping $\phi)$. Let $R$ be a square such that $\frac{1}{2} R$ is $h_{a}$-doubling. We say $R$ is of type $S$ if

$$
\mu\left(\bigcup_{\substack{Q \in \operatorname{Bad}(R): \\ \ell(Q) \geq \eta \ell(R)}} Q \cap \frac{1}{2} R\right) \geq \frac{1}{2} \mu\left(\frac{1}{2} R\right) .
$$

Otherwise, we say that $R$ is of type $L$. The letters $S$ and $L$ stand for "short" and "long" trees, respectively (this terminology will be more clear below).

Before constructing the families $\operatorname{Sel}(\mu), \operatorname{Sel}_{S}(\mu)$, and $\operatorname{Sel}_{L}(\mu)$, we have to define the family of terminal squares $\mathcal{T}(R)$.

5.4.1. Definition of $\mathcal{T}(R)$ when $R$ is of type S. Let $R$ be a square of type $S$, so that $\frac{1}{2} R$ is $h_{a}$-doubling. For $x \in 3 R$, consider the biggest 4 -dyadic square $Q_{x}$ of type $L$ containing $x$, such that $\frac{1}{2} Q_{x}$ is $h_{a}$-doubling, and such that $\ell\left(Q_{x}\right) \leq \ell(R) / 8$, if it exists. Let $\mathcal{T}_{0}(R)$ be the collection of these squares $Q_{x}$. We denote by $F(R)$ the subset of those points $x \in 3 R$ such there does not exists such a square $Q_{x}$.

By Vitali's covering theorem there exists a subfamily $\mathcal{T}(R) \subset \mathcal{T}_{0}(R)$ such that the squares $\{5 Q\}_{Q \in \mathcal{T}(R)}$ are pairwise disjoint and so that

$$
\bigcup_{Q \in \mathcal{T}_{0}(R)} 5 Q \subset \bigcup_{Q \in \mathcal{T}(R)} 15 Q
$$

Since the squares $Q_{x}$ that intersect $\frac{1}{2} R$ are contained in $R$ and they are doubling,

$$
\mu\left(\bigcup_{Q \in \mathcal{T}(R)} Q \cap R\right) \geq C_{7}^{-1} \mu\left(\frac{1}{2} R \backslash F(R)\right) .
$$

5.4.2. Definition of $\mathcal{T}(R)$ when $R$ is of type L. In this case

$$
\mu(G(R))+\mu\left(\bigcup_{\substack{Q \in \operatorname{Bad}(R): \\ \ell(Q)<\eta \ell(R)}} Q \cap \frac{1}{2} R\right) \geq \frac{1}{2} \mu\left(\frac{1}{2} R\right) .
$$

If $\mu(G(R)) \geq \frac{1}{4} \mu\left(\frac{1}{2} R\right)$, then we set $\mathcal{T}(R)=\varnothing$. 
Suppose now that $\mu(G(R))<\frac{1}{4} \mu\left(\frac{1}{2} R\right)$. Then,

$$
\mu\left(\bigcup_{\substack{Q \in \operatorname{Bad}(R): \\ \ell(Q)<\eta \ell(R)}} Q \cap \frac{1}{2} R\right) \geq \frac{1}{4} \mu\left(\frac{1}{2} R\right) .
$$

Recall that, the squares $C_{5} Q$ in Lemma 5.5 are doubling, by the statement (c) in the same lemma applied to $P=C_{5} Q$ (in fact, $\left(h_{a}, c\right)$ doubling, with $c=c(A, \delta)$, by Remark 5.6). We assume that the constant $\eta$ in the Definition 5.8 of L squares is small enough so that

$$
C_{5} \ell(Q) \leq \ell(R) / 100 \quad \text { if } \ell(Q)<\eta \ell(R),
$$

say. By Vitali's covering theorem, there exists a subfamily

$$
\left\{S_{j}\right\}_{j \in I_{R}} \subset\left\{C_{5} Q: Q \in \operatorname{Bad}(R), \ell(Q)<\eta \ell(R)\right\}
$$

such that the squares $5 S_{j}, j \in I_{R}$, are pairwise disjoint and contained in $R$ and, moreover, using the doubling property of the squares $C_{5} Q$,

$$
\mu\left(\bigcup_{j \in I_{R}} S_{j} \cap R\right) \geq C^{-1} \mu\left(\frac{1}{2} R\right) \geq C_{8}^{-1} \mu(R),
$$

with $C_{8}$ depending on $A, \delta$ (but not on $\eta$ ).

Take a square $S_{j}, j \in I_{R}$, such that $S_{j} \cap R \neq \varnothing$. For each $x \in E \cap S_{j}$, consider the biggest square $P_{x}$ centered at $x$, with $\ell\left(P_{x}\right) \leq \ell\left(S_{j}\right) / 16$, which is $\left(h_{a}, b\right)$-doubling, with $b$ as explained just above Remark 5.3, in case such a square exists. We denote by $F_{j}(R)$ the subset of those points $x \in E \cap S_{j}$ such there does not exists such a square. Denote by $\widehat{P}_{x}$ a 4 -dyadic square with side length $4 \ell\left(P_{x}\right)$ such that $P_{x} \subset \frac{1}{2} \widehat{P}_{x}$. Notice that the squares $\widehat{P}_{x}$ are $h_{a}$-doubling and they are contained in $3 S_{j}$.

By Vitali's covering theorem, there exists a subfamily $\left\{\widehat{P}_{x_{i}}\right\}_{i} \subset\left\{\widehat{P}_{x}\right\}_{x \in E \cap S_{j} \backslash F_{j}(R)}$ such that the squares $5 \widehat{P}_{x_{i}}$ are pairwise disjoint, and

$$
\mu\left(S_{j} \backslash F_{j}(R)\right) \leq C \mu\left(\bigcup_{i} \widehat{P}_{x_{i}}\right) .
$$

We define $\mathcal{T}_{j}(R):=\left\{\widehat{P}_{x_{i}}\right\}_{i}$, and finally

$$
\mathcal{T}(R):=\bigcup_{j \in I_{R}} \mathcal{T}_{j}(R)
$$

We also set

$$
F(R):=\bigcup_{j \in I_{R}} F_{j}(R)
$$


5.4.3. Definition of $\operatorname{Sel}(\mu), \operatorname{Sel}_{S}(\mu)$, and $\operatorname{Sel}_{L}(\mu)$. The family $\operatorname{Sel}(\mu)$ is constructed recursively. Let $R_{0}$ be a 4 -dyadic square with $\ell\left(R_{0}\right) \simeq \operatorname{diam}(E)$ such that $E$ is contained in one of the four dyadic squares in $\frac{1}{2} R_{0}$ with side length $\ell\left(R_{0}\right) / 4$. The first square of $\operatorname{Sel}(\mu)$ is $R_{0}$. The next squares that we choose as elements of $\operatorname{Sel}(\mu)$ are the ones from $\mathcal{T}\left(R_{0}\right)$. And, now the ones that belong to $\mathcal{T}(R)$ for some $R \in \mathcal{T}\left(R_{0}\right)$, an so on.

In other words, $\operatorname{Sel}(\mu)$ is the smallest family of 4-dyadic squares that contains $R_{0}$ and which has the property that if $R \in \operatorname{Sel}(\mu)$, then the squares from $\mathcal{T}(R)$ also belong to $\operatorname{Sel}(\mu)$.

The family $\operatorname{Sel}_{S}(\mu)$ is made up of the squares from $\operatorname{Sel}(\mu)$ of type $\mathrm{S}$, while $\operatorname{Sel}_{L}(\mu)$ is the subfamily of the squares from $\operatorname{Sel}(\mu)$ of type L.

\subsection{The packing condition for squares in $\operatorname{Tree}(R), R \in \operatorname{Sel}_{S}(\mu)$.}

Definition 5.9. For $R \in \operatorname{Sel}_{S}(\mu)$, we denote by $\operatorname{Term}(R)$ the collection of dyadic squares $Q$ such that $Q \subset 3 P$ for some $P \in \mathcal{T}_{0}(R)$, so that, moreover, $Q$ is maximal. We call them terminal squares.

We denote by $\operatorname{Tree}(R)$ the family of dyadic squares that are contained in $R$ and that are not properly contained in any square from $\operatorname{Term}(R)$.

We also set

$$
\operatorname{End}(R)=E \cap R \backslash \bigcup_{Q \in \operatorname{Term}(R)} P .
$$

Notice that the points in $\operatorname{End}(R)$ can be considered as terminal squares of $\operatorname{Tree}(R)$ with zero side length.

The main objective of this subsection consists in proving the following result.

Lemma 5.10. Let $R \in \operatorname{Sel}_{S}(\mu)$. Then,

$$
\sum_{Q \in \operatorname{Tree}(R)} \varepsilon_{a}(Q)^{2} \mu(Q) \leq C\left(A, \delta, \varepsilon_{0}, \eta\right) \mu(R) .
$$

The main tool for the proof will be the corona construction of [Tol05]. To state the precise result that we will use, we need to introduce some notation. Let $R$ be a 4-dyadic square such that $\frac{1}{2} R$ is $h_{a}$-doubling. The next lemma deals with a family $\operatorname{Top}_{R}(\mu)$ of 4-dyadic squares satisfying some precise properties. Given $Q \in \operatorname{Top}_{R}(\mu)$, we denote by $\operatorname{Stop}(Q)$ the subfamily of squares $P \in \operatorname{Top}_{R}(\mu)$ satisfying

(a) $P \cap 3 Q \neq \varnothing$,

(b) $\ell(P) \leq \ell(Q) / 8$,

(c) $P$ is maximal, in the sense that there are not other squares $\left\{P_{j}\right\}_{j} \subset \operatorname{Top}_{R}(\mu)$ with $\ell\left(P_{j}\right)<\ell(P)$ such that $P \subset \bigcup_{j} P_{j}$.

We also denote

$$
\widetilde{G}(Q)=3 Q \cap E \backslash \bigcup_{P \in \operatorname{Stop}(Q)} P .
$$


Lemma 5.11 ([Tol05]). Let $\mu$ be a Radon measure supported on $E \subset \mathbb{C}$ such that $\mu(B(x, r)) \leq r$ for all $x \in \mathbb{C}, r>0$, and $c^{2}\left(\mu_{\mid 40 R}\right)<\infty$. Let $\widetilde{A}>10$ be big enough and $\widetilde{\delta}, \widetilde{\varepsilon}_{0}>0$ small enough. Let $R$ be a 4 -dyadic square such that $\frac{1}{2} R$ is $h_{a}$-doubling. There exists a family $\operatorname{Top}_{R}(\mu)$ of 4-dyadic squares contained in $4 R$ such that

$$
\sum_{Q \in \operatorname{Top}_{R}(\mu)} \theta_{\mu}(Q)^{2} \mu(Q) \leq C\left(\widetilde{A}, \widetilde{\delta}, \widetilde{\varepsilon}_{0}\right)\left(\mu(R)+c^{2}\left(\mu_{\mid 40 R}\right)\right),
$$

and such that for $Q \in \operatorname{Top}_{R}(\mu)$, if $P$ is a square with $\ell(P) \leq \ell(Q)$ such that either $P \cap \widetilde{G}(Q) \neq \varnothing$ or there is another square $P^{\prime} \in \operatorname{Stop}(Q)$ satisfying $P \cap P^{\prime} \neq \varnothing$ and $\ell\left(P^{\prime}\right) \leq \ell(P)$, then

(a) $\theta_{\mu}(P) \leq C \widetilde{A} \theta_{\mu}(Q)$,

(b) every square $P^{\prime \prime}$ concentric with $P$ such that $P \subset P^{\prime \prime} \subset 5 R$ and $D_{\mu}\left(P, P^{\prime \prime}\right) \geq$ $C_{9}(\widetilde{A}, \widetilde{\delta}) \theta_{\mu}(Q)^{2}$, satisfies

$$
\theta_{\mu}\left(P^{\prime \prime}\right) \geq C^{-1} \widetilde{\delta} \theta_{\mu}(Q) .
$$

(c) every square $P^{\prime \prime}$ such that $\frac{1}{2} P^{\prime \prime}$ is $h_{a}$ doubling and $P \subset \frac{3}{4} P^{\prime \prime}, P^{\prime \prime} \subset 5 R$ and $D_{\mu}\left(P, P^{\prime \prime}\right) \geq C(\widetilde{A}, \widetilde{\delta}) \theta_{\mu}(Q)^{2}$, satisfies

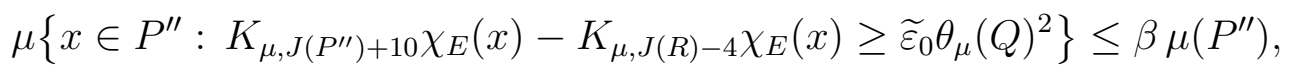

where $0<\beta<1$ is some fixed constant.

Some remarks about the choice of the constants $\widetilde{A}, \widetilde{\delta}, \widetilde{\varepsilon}_{0}, \beta$ in the preceding lemma are in order: first, $\widetilde{A}$ can be taken as big as desired. After choosing $\widetilde{A}$, one has to take $\widetilde{\delta} \leq \widetilde{\delta}_{1}(\widetilde{A})$, where $\widetilde{\delta}_{1}(\widetilde{A})$ is some fixed small constant, and finally, one has to choose $\widetilde{\varepsilon}_{0} \leq \widetilde{\varepsilon}_{1}(\widetilde{A}, \widetilde{\delta}, \beta)$. In particular, the preceding lemma holds for all $\widetilde{\varepsilon}_{0}$ small enough, at the price of increasing the constant in the right side of (5.7) as $\widetilde{\varepsilon}_{0} \rightarrow 0$.

In [Tol05, the reader will not find an exact statement such as Lemma 5.11. In fact, in [Tol05], every square $\frac{1}{2} Q$, with $Q \in \operatorname{Top}(\mu)$, is $(32,5000)$-doubling, instead of $h_{a}$-doubling. Also, Lemma 5.11 is proved only in the particular case where $E \subset R$. However, the same arguments, with very minor changes, work with the assumptions above. On the other hand, the corona decomposition of [Tol05] also states the existence of curves $\Gamma_{Q}$ satisfying properties similar to the ones of Lemma 5.5. However, this information is not useful to prove Lemma 5.10, and so we have skipped it.

Lemma 5.12. Let $R \in \operatorname{Sel}_{S}(\mu)$ and $Q_{0} \in \operatorname{Top}_{R}(\mu)$. Also, let $Q \in \operatorname{Tree}(R)$ be a 4-dyadic square such that $Q \cap 3 Q_{0} \neq \varnothing, \ell(Q) \leq \ell\left(Q_{0}\right) / 8$, and so that $\frac{1}{2} Q$ is $h_{a}$ doubling. Then there exists a collection of squares or points $\left\{P_{i}\right\}_{i}$ contained in $Q$ such that

(a) each $P_{i}$ is contained either in a union of squares from $P \in \operatorname{Term}(R) \cup \operatorname{End}(R)$, or in $3 P$, for some $P \in \operatorname{Stop}\left(Q_{0}\right)$,

(b) $D_{\mu}\left(P_{i}, Q\right) \leq M \theta_{\mu}(Q)^{2}$ if $\ell\left(P_{i}\right) \leq \ell(Q)$, with $M$ depending on $\widetilde{A}, \widetilde{\delta}$, 
(c) and

$$
\mu\left(Q \cap \bigcup_{i} P_{i}\right) \geq \tau \mu(Q),
$$

assuming that the constants $A, \widetilde{A}, \delta, \widetilde{\delta}, \varepsilon_{0}, \widetilde{\varepsilon}_{0}, \beta$ are chosen appropriately.

In this lemma, by convenience we understand that the points in $\operatorname{End}(R)$ are squares with zero side length. To prove it, we will make essential use of the fact that $R$ is of type $\mathrm{S}$.

Proof. If the square $Q$ is of type L, then $Q \in \operatorname{Term}(R)$ by definition (since $Q \in$ Tree $(R)$ and $R$ is of type $S$ ). Then we just take $P_{i}=Q$ and then the lemma holds. If every square $T$ which intersects $\frac{1}{2} Q$ and such that $D_{\mu}(T, Q) \geq M \theta_{\mu}(Q)^{2}$ is contained in $3 P$, for some $P \in \operatorname{Stop}\left(Q_{0}\right) \cup \widetilde{G}\left(Q_{0}\right)$, we are also done. Therefore, we may assume that $Q$ is of type $S$ and that there exists a square $T$ which intersects $\frac{1}{2} Q$ such that $D_{\mu}(T, Q) \geq M \theta_{\mu}(Q)^{2}$, satisfying $T \cap P \neq \varnothing$ for some $P \in \operatorname{Stop}\left(Q_{0}\right) \cup \widetilde{G}\left(Q_{0}\right)$ with $\ell(P)<\ell(T)$ (otherwise, $T \subset 3 P)$. This condition implies that

$$
C^{-1} \widetilde{\delta} \theta_{\mu}\left(Q_{0}\right) \leq \theta_{\mu}(Q) \leq C \widetilde{A} \theta_{\mu}\left(Q_{0}\right),
$$

by conditions (a) and (b) of Lemma 5.11, assuming $M$ big enough.

Since $Q$ is of type $\mathrm{S}$, there are squares $S_{i} \in \operatorname{Bad}(Q)$ such that $\eta \ell(Q) \leq \ell\left(S_{i}\right) \leq$ $\ell(Q) / 8$, with $S_{i} \cap \frac{1}{2} Q \neq \varnothing$, and

$$
\mu\left(\bigcup_{i} S_{i}\right) \geq \frac{1}{2} \mu\left(\frac{1}{2} Q\right) .
$$

By Lemma 5.7, if $\delta$ is small enough, there are squares $\left\{S_{i}\right\}_{i \in I_{H D}} \subset H D(Q)$ and $\left\{S_{i}\right\}_{i \in I_{H C}} \subset H C(Q)$ such that

$$
\mu\left(\bigcup_{i \in I_{H} \cup I_{H C}} S_{i}\right) \geq \frac{1}{4} \mu\left(\frac{1}{2} Q\right) .
$$

Notice that if $S_{i} \in H D(Q)$, then

$$
\theta_{\mu}\left(S_{i}\right) \geq C^{-1} A \theta_{\mu}(Q) \geq C^{-1} A \widetilde{\delta} \theta_{\mu}\left(Q_{0}\right) \gg \theta_{\mu}\left(Q_{0}\right)
$$

if we choose $A$ such that $A \widetilde{\delta} \gg \widetilde{A}$. Then it is easy to check that $S_{i}$ satisfies the conditions (a) and (b) of the lemma. Condition (c) also holds if

$$
\mu\left(\bigcup_{i \in I_{H} D} S_{i}\right) \geq \frac{1}{8} \mu\left(\frac{1}{2} Q\right) .
$$

If the latter condition fails, then we have

$$
\mu\left(\bigcup_{i \in I_{H C}} S_{i}\right) \geq \frac{1}{8} \mu\left(\frac{1}{2} Q\right) .
$$


Let $\left\{\widehat{P}_{j}\right\}_{j}$ be a family of 4-dyadic squares or points such that $\frac{1}{2} \widehat{P}_{j} h_{a}$-doubling for all $j$, which cover $\bigcup_{i \in I_{H C}} S_{i}$ with finite overlap, with $\ell\left(\widehat{P}_{j}\right) \leq \ell(Q) / 100$, so that

$$
D_{\mu}\left(\widehat{P}_{j}, Q\right) \leq C(\eta) \theta_{\mu}(Q)^{2} .
$$

By Tchebytchev, it is easy to check that there exists a subfamily $\left\{\widehat{P}_{j}\right\}_{j \in J} \subset\left\{\widehat{P}_{j}\right\}_{j}$ such that for each $j \in J$,

$$
\mu\left\{x \in P_{j}: K_{\mu, J\left(P_{j}\right)+10} \chi_{E}(x)-K_{\mu, J(Q)-4} \chi_{E}(x) \geq \varepsilon_{0} \theta_{\mu}(Q)^{2}\right\} \geq C_{10}^{-1} \mu\left(P_{j}\right),
$$

with

$$
\mu\left(\bigcup_{j \in J} \widehat{P}_{j}\right) \geq C^{-1} \mu(Q)
$$

Notice that for $x$ in a big piece of each square $\widehat{P}_{j}, j \in J$,

$$
\begin{aligned}
K_{\mu, J\left(\widehat{P}_{j}\right)+10} \chi_{E}(x)-K_{\mu, J\left(Q_{0}\right)-4} \chi_{E}(x) & \geq K_{\mu, J\left(\widehat{P}_{j}\right)+10} \chi_{E}(x)-K_{\mu, J(Q)-4} \chi_{E}(x) \\
& \geq \varepsilon_{0} \theta_{\mu}(Q)^{2} \geq C^{-1} \widetilde{\delta}^{2} \varepsilon_{0} \theta_{\mu}\left(Q_{0}\right)^{2} .
\end{aligned}
$$

Thus if we choose $\widetilde{\varepsilon}_{0}$ small enough so that $\widetilde{\varepsilon}_{0} \ll \widetilde{\delta}^{2} \varepsilon_{0}$, and we also take $\beta \ll C_{10}^{-1}$, then one can find squares $\left\{P_{i}^{j}\right\}_{i}$ contained in $\frac{3}{4} P_{j}$ which cover $\frac{1}{2} P_{j}$ with $D_{\mu}\left(P_{i}^{j}, P_{j}\right)=$ $C \theta_{\mu}\left(Q_{0}\right)$, so that the family $\bigcup_{j \in J}\left\{P_{i}^{j}\right\}_{i}$ satisfies all the required properties. We leave the details for the reader.

For $Q \in \operatorname{Top}_{R}(\mu)$, we denote by $\operatorname{Tree}_{R}(Q)$ the family of dyadic squares from Tree $(R)$ that are contained in $3 Q$ and contain either some of the sixteen dyadic squares of equal length that form one square from $\operatorname{Stop}(Q)$, or some point from $\widetilde{G}(Q)$.

Lemma 5.13. Given $R \in \operatorname{Sel}_{S}(\mu)$, for each $Q \in \operatorname{Top}_{R}(\mu)$,

$$
\sum_{P \in \operatorname{Tree}_{R}(Q)} \varepsilon_{a}(P)^{2} \mu(P) \leq C\left(A, \delta, \varepsilon_{0}, \eta\right) \varepsilon_{a}(Q)^{2} \mu(Q) .
$$

Recall that $\operatorname{Tree}_{R}(Q) \subset \operatorname{Tree}(R)$. Thus, in a sense all the relevant squares in the sum above are of type $S$, which originate short trees.

Proof. For $x \in \mathbb{C}$ we define the function

$$
F(x)=\sum_{k \in \mathbb{Z}} \max _{P \sim(x, k)} \varepsilon_{a}(P)^{2},
$$

where the notation $P \sim(x, k)$ means that $P$ is a 4-dyadic square containing $x$, with $\ell(P)=2^{-k}$ such that some of the 16 dyadic squares of equal side length that form $P$ belongs to $\operatorname{Tree}(Q)$. From the definition, it is easy to check that $F(x)=0$ if $x \notin C Q$, for some fixed $C>1$. To prove the lemma we will show that $\|F\|_{L^{1}(\mu)} \leq C \varepsilon_{a}(Q)^{2} \mu(Q)$.

For $\lambda>0$, denote

$$
\Omega_{\lambda}=\left\{x \in \mathbb{C}: F(x)>\lambda \varepsilon_{a}(Q)^{2}\right\} .
$$


For $x \in \Omega_{\lambda}$, let $k_{x}$ be the minimal integer such that

$$
\sum_{k \leq k_{x}} \max _{P \sim(x, k)} \varepsilon_{a}(P)^{2}>\lambda \varepsilon_{a}(Q)^{2}
$$

and let $\widetilde{S}_{x} \sim\left(x, k_{x}\right)$ be such that $\varepsilon_{a}\left(\widetilde{S}_{x}\right)$ is maximal. Let $S_{x}$ be the smallest 4-dyadic square such that $\frac{1}{2} S_{x}$ is $h_{a}$-doubling and contains $\widetilde{S}_{x}$. If $\ell\left(S_{x}\right)>\ell(Q)$, from Lemma 5.2, it follows easily that

$$
D_{\mu}\left(\widetilde{S}_{x}, Q\right) \leq C_{11} \varepsilon_{a}(Q)^{2}
$$

where $C_{11}$ may depend on $\widetilde{A}, \widetilde{\delta} \ldots$ This implies that

$$
F(x) \leq C_{12} \varepsilon_{a}(Q)^{2},
$$

with $C_{12}$ depending on $C_{11}$.

Assume that $\lambda>C_{12}$. In this case, $\ell\left(S_{x}\right) \leq \ell(Q)$. From Lemma 5.2 and the fact that for all $P \in \operatorname{Tree}_{R}(Q), \varepsilon_{a}(P) \leq C(\widetilde{A}) \varepsilon_{a}(Q)$, one infers that

$$
D_{\mu}\left(\widetilde{S}_{x}, S_{x}\right) \leq C_{11} \varepsilon_{a}(Q)^{2}
$$

From this estimate, one deduces that

$$
F(y)>\left(\lambda-C_{13}\right) \varepsilon_{a}(Q)^{2} \quad \text { for all } y \in S_{x},
$$

with $C_{13}$ depending on $C_{11}$. So we have

$$
\Omega_{\lambda} \subset \bigcup_{x} S_{x} \subset \Omega_{\lambda-C_{13}}
$$

From the doubling properties of the squares $S_{x}$, there exists a subfamily $\left\{S_{x_{i}}\right\}$ such that the squares from this family are pairwise disjoint and

$$
\mu\left(\bigcup_{i} S_{x_{i}}\right) \geq C^{-1} \mu\left(\bigcup_{x} S_{x}\right) .
$$

We may cover each square $\frac{1}{2} S_{x_{i}}$ with a family of squares $\left\{T_{j}^{i}\right\}_{j}$ such that each $\frac{1}{2} T_{j}^{i}$ is $h_{a}$-doubling. By Lemma 5.12, for each $T_{j}^{i}$ there exists some subset $A_{j}^{i}$ such that $\mu\left(A_{j}^{i}\right) \geq C^{-1} \mu\left(T_{j}^{i}\right)$ and

$$
F(x) \leq \lambda+C
$$

(because of (b) in Lemma 5.12 and because $D_{\mu}\left(T_{j}^{i}, S_{x_{i}}\right) \leq C$ ). Using some appropriate covering theorem (like Vitali), one infers that for each $i$ there exists $A_{i} \subset S_{x_{i}}$ such that $\mu\left(A_{i}\right) \geq C^{-1} \mu\left(S_{x_{i}}\right)$ and $F(x) \leq \lambda+C_{14}$ on $A_{i}$.

Since $A_{i} \subset \Omega_{\lambda-C_{13}} \backslash \Omega_{\lambda+C_{14}}$, we deduce

$$
\mu\left(\Omega_{\lambda-C_{13}}\right)-\mu\left(\Omega_{\lambda+C_{14}}\right) \geq \sum_{i} \mu\left(A_{i}\right) \geq C^{-1} \sum_{i} \mu\left(S_{x_{i}}\right) \geq C_{15}^{-1} \mu\left(\Omega_{\lambda}\right) .
$$

Thus,

$$
\mu\left(\Omega_{\lambda+C_{14}}\right) \leq \mu\left(\Omega_{\lambda-C_{13}}\right)-C_{15}^{-1} \mu\left(\Omega_{\lambda}\right)
$$


We have

$$
\|F\|_{L^{1}(\mu)} \leq \varepsilon_{a}(Q)^{2} \int_{0}^{\infty} \mu\left(\Omega_{\lambda}\right) d \lambda \leq C_{12} \varepsilon_{a}(Q)^{2} \mu(C Q)+\varepsilon_{a}(Q)^{2} \int_{C_{12}}^{\infty} \mu\left(\Omega_{\lambda}\right) d \lambda
$$

We may assume that $C_{12}, C_{13}, C_{14}$ are integer constants. Then,

$$
\int_{C_{12}}^{\infty} \mu\left(\Omega_{\lambda}\right) d \lambda \leq \sum_{k \geq C_{12}} \mu\left(\Omega_{k}\right)
$$

From (5.10), one can easily check that $\mu\left(\Omega_{k}\right)$ decreases geometrically as $k \rightarrow \infty$. Indeed, (5.10) this implies that

$$
\mu\left(\Omega_{\lambda+C_{14}}\right)\left(1+C_{15}^{-1}\right) \leq \mu\left(\Omega_{\lambda-C_{13}}\right) .
$$

That is, $\mu\left(\Omega_{\lambda+C_{14}+C_{13}}\right) \leq \alpha \mu\left(\Omega_{\lambda}\right)$, with $\alpha=\left(1+C_{15}^{-1}\right)^{-1}$. Then we deduce that

$$
\sum_{k \geq C_{12}} \mu\left(\Omega_{k}\right) \leq C \mu(Q)
$$

and then the lemma follows.

Proof of Lemma 5.10. Let $\operatorname{Top}_{R}(\mu)$ be the family described in Lemma 5.11. Since $c_{\mu}^{2}(x) \leq 1$ for all $x \in \mathbb{C}$, we have

$$
\sum_{Q \in \operatorname{Top}_{R}(\mu)} \theta_{\mu}(Q)^{2} \mu(Q) \leq C\left(\widetilde{A}, \widetilde{\delta}, \widetilde{\varepsilon}_{0}\right) \mu(R) .
$$

Notice that

$$
\operatorname{Tree}(R) \subset \bigcup_{Q \in \operatorname{Top}_{R}(\mu)} \operatorname{Tree}_{R}(Q) .
$$

By the preceding lemma, for each $Q \in \operatorname{Top}_{R}(\mu)$,

$$
\sum_{P \in \operatorname{Tree}_{R}(Q)} \varepsilon_{a}(P)^{2} \mu(P) \leq C\left(A, \delta, \varepsilon_{0}, \eta\right) \varepsilon_{a}(Q)^{2} \mu(Q) .
$$

Together with (5.11) and the fact that $\varepsilon_{a}(Q) \approx \theta_{\mu}(Q)$ for $Q \in \operatorname{Top}_{R}(\mu)$, this yields

$$
\begin{aligned}
\sum_{P \in \operatorname{Tree}(R)} \varepsilon_{a}(P)^{2} \mu(P) & \leq \sum_{Q \in \operatorname{Top}_{R}(\mu)} \sum_{P \in \operatorname{Tree}_{R}(Q)} \varepsilon_{a}(P)^{2} \mu(P) \\
& \leq C \sum_{Q \in \operatorname{Top}_{R}(\mu)} \varepsilon_{a}(Q)^{2} \mu(Q) \leq C_{16} \mu(R),
\end{aligned}
$$

with $C_{16}$ depending on all the parameters $\eta, A, \delta, \varepsilon_{0}$. 


\section{Construction of the measure $\nu$ For the proof of Theorem 1.1}

In this section we will prove the estimate (3.1) following the ideas explained in Section 3. To this end, using the corona decomposition of the preceding section we will construct a measure $\nu$ supported on $\phi(E)$ such that $\nu(\phi(E)) \approx \gamma(E)^{\frac{2 K}{K+1}}$ and $\dot{W}_{\frac{2 K}{2 K+1}}^{\nu}, \frac{2 K+1}{K+1}(x) \lesssim 1$ for all $x \in \phi(E)$.

6.1. Preliminaries. Next lemma is just a rescaled version of Lemma 2.11

Lemma 6.1. Let $\mu$ be a finite continuous (i.e. without point masses) Borel measure on $\mathbb{C}$. For $a>0$ small enough (depending only on $K$ ), denote

$$
\varepsilon_{a}(x, t)=\varepsilon_{a}(B)=\frac{1}{t} \int \psi_{a}\left(\frac{y-x}{t}\right) d \mu(y), \quad h_{a}(x, t)=t \varepsilon_{a}(x, t),
$$

with $\psi_{a}$ as in (2.2). Let $\phi: \mathbb{C} \rightarrow \mathbb{C}$ be a $K$-quasiconformal mapping and set

$$
\varepsilon(x, t)=\varepsilon_{a}\left(\phi^{-1}(B(x, t))\right)^{\frac{2 K}{K+1}}, \quad h(x, t)=t^{\frac{2}{K+1}} \varepsilon(x, t) .
$$

If $E \subset \mathbb{C}$ is a compact subset contained in a ball $B$, we have

$$
\frac{M^{h_{a}}(E)}{\operatorname{diam}(B)} \leq C(K)\left(\frac{M^{h}(\phi(E))}{\operatorname{diam}(\phi(B))^{\frac{2}{K+1}}}\right)^{\frac{K+1}{2 K}} .
$$

Obviously, an analogous version holds with squares instead of balls.

Lemma 6.2. Under the same hypotheses and notation of Lemma 6.1, given any square $Q \subset \mathbb{C}$, if

with $C_{17}>0$, then

$$
M^{h_{a}}(Q \cap E) \geq C_{17} h_{a}(Q)
$$

$$
M^{h}(\phi(Q \cap E)) \geq C_{18} h(\phi(Q)),
$$

with $C_{18}>0$ depending only on $C_{17}$ and $K$.

Proof. We have

$$
\frac{M^{h_{a}}(Q \cap E)}{\ell(Q)} \geq C \varepsilon_{a}(Q) .
$$

Then, by Lemma 6.1 applied to $Q \cap E$ and $Q$ instead of $B$,

$$
\frac{M^{h}(\phi(Q \cap E))}{\operatorname{diam}(\phi(Q))^{\frac{2}{K+1}}} \geq C \varepsilon_{a}(Q)^{\frac{2 K}{K+1}},
$$

which is equivalent to $M^{h}(\phi(Q \cap E)) \geq C_{18} h(\phi(Q))$.

Notice that the assumption $M^{h_{a}}(Q \cap E) \geq C_{17} h_{a}(Q)$ is satisfied by the squares from $\operatorname{Sel}(\mu)$ in the corona construction in Section 5 , since

$$
M^{h_{a}}(Q \cap E) \geq C \mu(Q) \geq C h_{a}(Q),
$$

by Lemma 2.2 and $Q$ is $h_{a}$-doubling. 
To construct $\nu$ we will use the structure of 4-dyadic squares from $\operatorname{Sel}(\mu)$ introduced in the preceding section. We denote $\operatorname{Sel}(\nu):=\phi(\operatorname{Sel}(\mu))$, and analogously for other families of squares such as $\operatorname{Sel}_{S}(\nu), \operatorname{Sel}_{L}(\nu)$, etc. Given a 4-dyadic $\phi$-square $R \in \operatorname{Sel}(\nu)$, we denote $\mathcal{T}_{\nu}(R):=\phi\left(\mathcal{T}\left(\phi^{-1}(R)\right)\right)$ and $F_{\nu}(R):=\phi\left(F\left(\phi^{-1}(R)\right)\right.$ (see Subsections 5.4.1 and 5.4.2), and also $G_{\nu}(R):=\phi\left(G\left(\phi^{-1}(R)\right)\right.$ (see (5.5)).

We will define the values of $\nu$ on the squares of $\operatorname{Sel}(\nu)$ (and/or other subsets like $G_{\nu}(R)$ or $F_{\nu}(R)$, for $\left.R \in \operatorname{Sel}(\nu)\right)$ inductively. To start with, we set

$$
\nu\left(\phi\left(R_{0}\right)\right)=M^{h}(\phi(E)) .
$$

Recall that $R_{0}$ is the biggest 4 -dyadic square from $\operatorname{Sel}(\mu)$, so that $E$ is contained in one of the 4 dyadic squares that form $\frac{1}{2} R_{0}$.

In the algorithm of construction of $\nu$, after fixing $\nu(R)$ for some $R \in \operatorname{Sel}(\nu)$, then one defines the values of $\nu(P)$ for all $P \in \mathcal{T}_{\nu}(R)$, as well as in $G(R) \cup F(R)$. To this end, it is necessary to distinguish two cases, according to wether $R$ is of type $\mathrm{L}$ or S. In Subsection 6.2 we consider the case where $R$ is of type L, and in Subsection 6.3, the one where $R$ is of type $\mathrm{S}$.

To simplify notation, in the rest of the paper given a square $Q$, we denote $Q^{\prime}=$ $\phi(Q)$. Usually, the letters $P, Q, R$ will be reserved for squares, and $P^{\prime}, Q^{\prime}, R^{\prime}$ for $\phi$-squares.

6.2. Definition of $\nu$ on $\mathcal{T}_{\nu}\left(R^{\prime}\right)$ when $R^{\prime} \in \operatorname{Sel}_{L}(\nu)$. Suppose first that

$$
\mu(G(R))<\frac{1}{4} \mu\left(\frac{1}{2} R\right)
$$

6.2.1. First step: definition of $\left.\nu\left(3 S_{j}^{\prime}\right)\right), j \in I_{R}$. Recall the definition of the squares $S_{j}, j \in I_{R}$, in (5.6). In particular, recall that the squares $5 S_{j}, j \in I_{R}$, are pairwise disjoint, contained in $R$, so that $S_{j} \cap \Gamma_{R} \neq \varnothing$ (where $\Gamma_{R}$ is a chord arc curve or a union of at most $N_{0}$ chord arc curves), and moreover,

$$
\sum_{j \in I_{R}} \mu\left(S_{j}\right) \geq C^{-1} \mu(R)
$$

We have

$$
\sum_{j \in I_{R}} \ell\left(S_{j}\right) \geq C_{19} \operatorname{diam}\left(\Gamma_{R}\right)
$$

with $C_{19}$ depending on $A, \delta$ (but not on $\eta$ ), because by the property (c) from Lemma 5.5,

$$
\sum_{j \in I_{R}} \ell\left(S_{j}\right)=\sum_{j \in I_{R}} \mu\left(S_{j}\right) \theta_{\mu}\left(S_{j}\right)^{-1} \approx \sum_{j \in I_{R}} \mu\left(S_{j}\right) \theta_{\mu}(R) \geq C^{-1} \mu(R) \theta_{\mu}(R)=C \ell(R) .
$$

Then, from Lemma 4.5 applied to suitable arcs contained in $3 S_{j} \cap \Gamma_{R}$ and quasisymmetry we deduce

$$
\sum_{j \in I_{R}} \ell\left(S_{j}^{\prime}\right)^{\alpha} \geq C_{20} \operatorname{diam}(\phi(\Gamma))^{\alpha} \approx \ell\left(R^{\prime}\right)^{\alpha}
$$


where $\alpha>2 /(K+1)$ depends only on $K$, and $C_{20}$ depends on $C_{19}, K$, and the parameters of the corona construction (except $\eta$ ). In fact, a similar argument shows that the set $G^{\prime}:=\bigcup_{j \in I_{R}} 3 S_{j}^{\prime}$ satisfies

$$
H_{\infty}^{\alpha}\left(G^{\prime}\right) \geq C_{21} \operatorname{diam}(\phi(\Gamma))^{\alpha} .
$$

To see this, just take into account that any union of sub-arcs of $\Gamma_{R}$ that contains $\Gamma_{R} \cap \bigcup_{j \in I_{R}} 3 S_{j}$ satisfies an estimate analogous to (6.2), and thus we would get an estimate similar to (6.3) for the corresponding images.

By Frostman Lemma, we deduce that there exists some measure $\sigma$ supported on $G^{\prime}$ such that $\sigma\left(G^{\prime}\right)=H_{\infty}^{\alpha}\left(G^{\prime}\right)$ and

$$
\sigma(B(x, r)) \leq C r^{\alpha} \quad \text { for all } x \in \mathbb{C}, r>0 .
$$

We define

$$
\nu\left(3 S_{j}^{\prime}\right)=\frac{\sigma\left(3 S_{j}^{\prime}\right)}{\sigma\left(G^{\prime}\right)} \nu\left(R^{\prime}\right)
$$

(recall that we assume that $\nu\left(R^{\prime}\right)$ has already been fixed), and moreover,

$$
\nu\left(5 S_{j}^{\prime} \backslash 3 S_{j}^{\prime}\right)=0 .
$$

It is easy to check then that if $P^{\prime}$ is a $\phi$-square concentric with $S_{j}^{\prime}$ which contains $3 S_{j}^{\prime}$ and is contained in $3 R^{\prime}$, then

$$
\nu\left(P^{\prime}\right) \leq C \frac{\ell\left(P^{\prime}\right)^{\alpha}}{\sigma\left(G^{\prime}\right)} \nu\left(R^{\prime}\right) \approx \frac{\ell\left(P^{\prime}\right)^{\alpha}}{\ell\left(R^{\prime}\right)^{\alpha}} \nu\left(R^{\prime}\right) .
$$

This can be proved using the condition (6.4) and the fact that the $\phi$-squares $5 S_{j}^{\prime}$ are disjoint, for instance. Therefore,

$$
\frac{\nu\left(P^{\prime}\right)}{\ell\left(P^{\prime}\right)^{\frac{2}{K+1}}} \leq C\left(\frac{\ell\left(P^{\prime}\right)}{\ell\left(R^{\prime}\right)}\right)^{\alpha-\frac{2}{K+1}} \frac{\nu\left(R^{\prime}\right)}{\ell\left(R^{\prime}\right)^{\frac{2}{K+1}}} .
$$

6.2.2. Second step: definition of $\nu\left(P^{\prime}\right)$ for $P^{\prime} \in \mathcal{T}_{\nu}\left(R^{\prime}\right)$. Recall that for each $j \in I_{R}$, there is a family $\mathcal{P}^{\prime}=\mathcal{T}_{j, \nu}\left(R^{\prime}\right) \cup F_{j}\left(R^{\prime}\right)$ of $\phi$-squares or points $P^{\prime}$ which are contained in $3 S_{j}^{\prime}$, such that different $\phi$-squares $5 P^{\prime}$ are pairwise disjoint and

$$
\mu\left(\bigcup_{P \in \mathcal{P}} P\right) \geq C^{-1} \mu\left(5 S_{j}\right) .
$$

We denote

$$
G_{j}^{\prime}=\bigcup_{P^{\prime} \in \mathcal{P}^{\prime}} P^{\prime}
$$

Our next task consists in distributing the measure $\nu_{\mid 3 S_{j}^{\prime}}$ among the $\phi$-squares $P^{\prime}$ above. In particular, we will have

$$
\nu\left(3 S_{j}^{\prime}\right)=\nu\left(G_{j}^{\prime}\right) .
$$


To define the appropriate values of $\nu\left(P^{\prime}\right)$, for $P^{\prime} \in \mathcal{P}^{\prime}$, we will follow an algorithm inspired by the proof of Frostman Lemma "from above". Let $Q_{0}^{\prime}$ be a dyadic $\phi$ square contained in $3 S_{j}^{\prime}$, with $\ell\left(Q_{0}\right)=\ell\left(S_{j}^{\prime}\right)$, such that $\mu\left(Q_{0}^{\prime} \cap G_{j}^{\prime}\right)$ is maximal. We set

$$
\tau\left(Q_{0}^{\prime}\right)=\nu\left(3 S_{j}^{\prime}\right)
$$

where $\tau$ should be considered as a preliminary version of $\nu$ on some $\phi$-squares contained in $3 S_{j}^{\prime}$. If $Q^{\prime}$ is a dyadic $\phi$-square contained in $Q_{0}^{\prime}$ such that $\tau\left(Q^{\prime}\right)$ has already been defined and $Q^{\prime}$ is not contained in any $\phi$-square from $\mathcal{P}^{\prime}$ (in particular, $Q^{\prime} \notin \mathcal{P}^{\prime}$ ), then we define $\tau$ on the sons $Q_{1}^{\prime}, \ldots, Q_{4}^{\prime}$ of $Q^{\prime}$ as follows:

$$
\tau\left(Q_{i}^{\prime}\right)=\frac{M^{h}\left(Q_{i}^{\prime} \cap G_{j}^{\prime}\right)}{\sum_{k=1}^{4} M^{h}\left(Q_{k}^{\prime} \cap G_{j}^{\prime}\right)} \tau\left(Q^{\prime}\right) .
$$

Clearly, we have $\sum_{1 \leq i \leq 4} \tau\left(Q_{i}^{\prime}\right)=\tau\left(Q^{\prime}\right)$. At the end of the algorithm, for each $P^{\prime} \in \mathcal{P}^{\prime}$ there is a pairwise disjoint family of dyadic $\phi$-squares $T_{1}^{\prime}, \ldots, T_{m}^{\prime}$ such that $P^{\prime}=$ $\bigcup_{1 \leq i \leq m} T_{i}^{\prime}$ so that $\tau\left(T_{i}^{\prime}\right)$ has been defined. We set

$$
\nu\left(P^{\prime}\right)=\sum_{1 \leq i \leq m} \tau\left(T_{i}^{\prime}\right)
$$

6.2.3. The case $\mu(G(R)) \geq \frac{1}{4} \mu\left(\frac{1}{2} R\right)$. The arguments for this case are very similar to the ones of Subsection 6.2.1. Instead of $\phi$-squares $S_{j}^{\prime}$, we have now points from $G_{\nu}\left(R^{\prime}\right)$. So we construct $\nu_{\mid 3 R^{\prime}}$ so that it is concentrated on $G_{\nu}\left(R^{\prime}\right)$, arguing as in Subsection 6.2.1. We leave the details for the reader.

\subsection{Definition of $\nu$ on $\mathcal{T}_{\nu}\left(R^{\prime}\right)$ when $R^{\prime} \in \operatorname{Sel}_{S}(\nu)$.}

6.3.1. The case $\mu(F(R)) \leq \frac{1}{4} \mu\left(\frac{1}{2} R\right)$. Recall the definition of the family of squares $\mathcal{T}(R)$. For $P \in \mathcal{T}(R)$, Set

$$
U(P)=\sum_{Q \in \mathcal{D}: P \subset Q \subset R} \varepsilon_{a}(Q)^{2}=\sum_{Q^{\prime} \in \phi \mathcal{D}: P^{\prime} \subset Q^{\prime} \subset R^{\prime}} \varepsilon\left(Q^{\prime}\right)^{\frac{K+1}{K}} .
$$

By Lemma 5.10 ,

$$
\sum_{P \in \mathcal{T}(R)} U(P) \mu(P) \leq C(\eta) \mu(R) .
$$

Since $\mu\left(\bigcup_{P \in \mathcal{T}(R)} P\right) \approx \mu(R)$, by Tchebytchev there is a subfamily $\mathcal{T}_{1}(R) \subset \mathcal{T}(R)$ such that

$$
\mu\left(\bigcup_{P \in \mathcal{T}_{1}(R)} P\right) \approx \mu(R) \quad \text { and } \quad U(P) \leq 2 C(\eta) \quad \text { for every } P \in \mathcal{T}_{1}(R) .
$$

For $P^{\prime} \in \mathcal{T}_{\nu}\left(R^{\prime}\right) \backslash \mathcal{T}_{1, \nu}\left(R^{\prime}\right)$, we set

$$
\nu\left(P^{\prime}\right)=0 .
$$


To define $\nu$ on the $\phi$-squares from $\mathcal{T}_{1, \nu}\left(R^{\prime}\right)$ we follow the same algorithm of Subsection 6.2.2, we denote

$$
G^{\prime}=\bigcup_{P^{\prime} \in \mathcal{T}_{1, \nu}\left(R^{\prime}\right)} P^{\prime} .
$$

Let $Q_{0}$ one of the 16 dyadic squares that form $R$ such that $\mu\left(Q_{0} \cap G\right)$ is maximal. We set

$$
\tau\left(Q_{0}^{\prime}\right)=\nu\left(R^{\prime}\right)
$$

where $\tau$ should be considered as a preliminary version of $\nu$ on some $\phi$-squares contained in $R$. If $Q^{\prime}$ is a dyadic $\phi$-square contained in $Q_{0}^{\prime}$ such that $\tau\left(Q^{\prime}\right)$ has already been defined and $Q^{\prime}$ is not contained in any $\phi$-square from $\mathcal{T}_{\nu, 1}\left(R^{\prime}\right)$ (in particular, $\left.Q^{\prime} \notin \mathcal{T}_{\nu, 1}\left(R^{\prime}\right)\right)$, then we define $\tau$ on the sons $Q_{1}^{\prime}, \ldots, Q_{4}^{\prime}$ of $Q^{\prime}$ as follows:

$$
\tau\left(Q_{i}^{\prime}\right)=\frac{M^{h}\left(Q_{i}^{\prime} \cap G^{\prime}\right)}{\sum_{k=1}^{4} M^{h}\left(Q_{k}^{\prime} \cap G^{\prime}\right)} \tau\left(Q^{\prime}\right) .
$$

Clearly, we have $\sum_{1 \leq i \leq 4} \tau\left(Q_{i}^{\prime}\right)=\tau\left(Q^{\prime}\right)$. At the end of the algorithm, for each $P^{\prime} \in$ $\mathcal{T}_{\nu, 1}\left(R^{\prime}\right)$ there is a pairwise disjoint family of dyadic $\phi$-squares $T_{1}^{\prime}, \ldots, T_{m}^{\prime}$ such that $P^{\prime}=\bigcup_{1 \leq i \leq m} T_{i}^{\prime}$ so that $\tau\left(T_{i}^{\prime}\right)$ has been defined. We set

$$
\nu\left(P^{\prime}\right)=\sum_{1 \leq i \leq m} \tau\left(T_{i}^{\prime}\right)
$$

6.3.2. The case $\mu(F(R)) \geq \frac{1}{4} \mu\left(\frac{1}{2} R\right)$. This case is treated as the preceding one, with the convention that the points from $F(R)$ are the same as squares with zero side length.

6.4. Estimate of the Wolff potential of $\nu$ on trees of type L. Recall that $\eta$ is the constant in the Definition 5.8 of short and long trees, and that $\eta \ll 1$.

Lemma 6.3. Let $R^{\prime} \in \operatorname{Sel}_{L}(\nu)$. If $\nu\left(R^{\prime}\right) \leq b h\left(R^{\prime}\right)$, then

$$
\nu\left(P^{\prime}\right) \leq C_{22} b \eta^{\alpha-\frac{2}{K+1}} h\left(P^{\prime}\right) \quad \text { for all } P^{\prime} \in \mathcal{T}_{\nu}\left(R^{\prime}\right) .
$$

Also, if $Q^{\prime}$ is a $\phi$-square such that $P^{\prime} \subset Q^{\prime} \subset 3 R^{\prime}$ for some $P^{\prime} \in \mathcal{T}_{\nu}\left(R^{\prime}\right) \cup G_{\nu}\left(R^{\prime}\right) \cup$ $F_{\nu}\left(R^{\prime}\right)$, then

$$
\nu\left(Q^{\prime}\right) \leq C_{22} b h\left(Q^{\prime}\right)
$$

Moreover, for each $P^{\prime} \in \mathcal{T}_{\nu}\left(R^{\prime}\right) \cup G_{\nu}\left(R^{\prime}\right) \cup F_{\nu}\left(R^{\prime}\right)$

$$
\sum_{Q^{\prime} \in \phi \mathcal{D}: P^{\prime} \subset Q^{\prime} \subset R^{\prime}}\left(\frac{\nu\left(3 Q^{\prime}\right)}{\ell\left(Q^{\prime}\right)^{\frac{2}{K+1}}}\right)^{\frac{K+1}{K}} \leq C b^{\frac{K+1}{K}} .
$$


Let us remark that the constant $C_{22}$ is independent from $\eta$.

One of the key points in this lemma is that, by (6.10),

$$
\frac{\nu\left(P^{\prime}\right)}{h\left(P^{\prime}\right)} \ll \frac{\nu\left(R^{\prime}\right)}{h\left(R^{\prime}\right)}
$$

if $P^{\prime} \in \mathcal{T}_{\nu}\left(R^{\prime}\right)$, for $R^{\prime} \in \operatorname{Sel}_{L}(\nu)$, assuming that $\eta$ is chosen small enough. This is due to improved distortion estimates for sub-arcs of chord arc curves. This point plays an essential role in our proof of Theorem 1.1.

Proof. For simplicity we will only consider the case where $\mu(G(R))<\mu\left(\frac{1}{2} R\right) / 4$, and that $\mu\left(F_{j}(R)\right) \leq \mu\left(S_{j}\right) / 2$ for all $j$. By arguments quite similar to the ones below, one can deal with the other cases, considering points as squares of zero side length.

Recall that if $Q^{\prime}$ is a $\phi$-square concentric with $S_{j}^{\prime}$ which contains $5 S_{j}^{\prime}$ and is contained in $3 R^{\prime}$, by (6.5),

$$
\frac{\nu\left(Q^{\prime}\right)}{\ell\left(Q^{\prime}\right)^{\frac{2}{K+1}}} \leq C\left(\frac{\ell\left(Q^{\prime}\right)}{\ell\left(R^{\prime}\right)}\right)^{\alpha-\frac{2}{K+1}} \frac{\nu\left(R^{\prime}\right)}{\ell\left(R^{\prime}\right)^{\frac{2}{K+1}}} \leq C b\left(\frac{\ell\left(Q^{\prime}\right)}{\ell\left(R^{\prime}\right)}\right)^{\alpha-\frac{2}{K+1}} \varepsilon\left(R^{\prime}\right),
$$

since $\nu\left(R^{\prime}\right) \leq b \ell\left(R^{\prime}\right)^{\frac{2}{K+1}} \varepsilon\left(R^{\prime}\right)$. By construction, $\varepsilon\left(Q^{\prime}\right) \approx \varepsilon\left(R^{\prime}\right)$ (by Remark 5.6) and so we get

$$
\nu\left(Q^{\prime}\right) \leq C b\left(\frac{\ell\left(Q^{\prime}\right)}{\ell\left(R^{\prime}\right)}\right)^{\alpha-\frac{2}{K+1}} \ell\left(Q^{\prime}\right)^{\frac{2}{K+1}} \varepsilon\left(Q^{\prime}\right)=C b\left(\frac{\ell\left(Q^{\prime}\right)}{\ell\left(R^{\prime}\right)}\right)^{\alpha-\frac{2}{K+1}} h\left(Q^{\prime}\right) .
$$

Recall that the subset $G_{j}^{\prime}=\bigcup_{P^{\prime} \in \mathcal{P}^{\prime}} P^{\prime}$ of $5 S_{j}^{\prime}$ and the $\phi$-square $Q_{0}^{\prime}$ in (6.6) satisfy

$$
M^{h_{a}}\left(Q_{0} \cap G_{j}\right) \geq C^{-1} \mu\left(G_{j}\right) \geq C^{-1} \mu\left(5 S_{j}\right) .
$$

Since $\varepsilon_{a}(R) \approx \varepsilon_{a}\left(5 S_{j}\right) \approx \theta_{\mu}\left(5 S_{j}\right)$, this implies

$$
M^{h_{a}}\left(Q_{0} \cap G_{j}\right) \geq C h_{a}\left(5 S_{j}\right)
$$

and then, by Lemma 6.2 ,

$$
M^{h}\left(Q_{0}^{\prime} \cap G_{j}^{\prime}\right) \geq C h\left(5 S_{j}^{\prime}\right)
$$

Thus, by (6.14),

$$
\tau\left(Q_{0}^{\prime}\right)=\nu\left(5 S_{j}^{\prime}\right) \leq C_{23} b \eta^{\alpha-\frac{2}{K+1}} M^{h}\left(G_{j}^{\prime} \cap Q_{0}^{\prime}\right) .
$$

We claim that all the numbers $\tau\left(Q^{\prime}\right)$ in (6.7) satisfy the analogous inequality

$$
\tau\left(Q^{\prime}\right) \leq C_{23} b \eta^{\alpha-\frac{2}{K+1}} M^{h}\left(G_{j}^{\prime} \cap Q^{\prime}\right)
$$

To prove this, it is enough to show that if this hods for some $\phi$-square $Q^{\prime}$, then it also holds for its sons $Q_{i}^{\prime}, 1 \leq i \leq 4$, assuming that $Q^{\prime}$ is not contained in any 
$\phi$-square from $\mathcal{P}^{\prime}$ (this was the necessary condition to define $\tau\left(Q_{i}^{\prime}\right), 1 \leq i \leq 4$ ). By (6.7), we get

$$
\begin{aligned}
\tau\left(Q_{i}^{\prime}\right) & =\frac{M^{h}\left(Q_{i}^{\prime} \cap G_{j}^{\prime}\right)}{\sum_{k=1}^{4} M^{h}\left(Q_{k}^{\prime} \cap G_{j}^{\prime}\right)} \tau\left(Q^{\prime}\right) \\
& \leq \frac{M^{h}\left(Q_{i}^{\prime} \cap G_{j}^{\prime}\right)}{M^{h}\left(Q^{\prime} \cap G_{j}^{\prime}\right)} \tau\left(Q^{\prime}\right) \leq C_{23} b \eta^{\alpha-\frac{2}{K+1}} M^{h}\left(Q_{i}^{\prime} \cap G_{j}^{\prime}\right),
\end{aligned}
$$

and so (6.15) holds. From this estimate one easily obtains

$$
\nu\left(Q^{\prime}\right) \leq C b \eta^{\alpha-\frac{2}{K+1}} M^{h}\left(Q_{i}^{\prime} \cap G_{j}^{\prime}\right)
$$

for $Q^{\prime}$ contained in $5 S_{j}^{\prime}$ and containing some $P_{0}^{\prime} \in \mathcal{T}\left(R^{\prime}\right)$. Indeed,

$$
\nu\left(Q^{\prime}\right) \leq \nu\left(\bigcup_{P^{\prime} \in \mathcal{T}_{\nu}\left(R^{\prime}\right): P^{\prime} \cap Q^{\prime} \neq \varnothing} P^{\prime}\right) .
$$

From the fact that the $\phi$-squares $5 P^{\prime}, P^{\prime} \in \mathcal{T}_{\nu}\left(R^{\prime}\right)$ are pairwise disjoint, it follows that if $Q^{\prime}$ intersects another $\phi$-square $P^{\prime} \in \mathcal{T}_{\nu}\left(R^{\prime}\right)$, then $\ell(Q) \geq \ell(P)$. As a consequence, all $\phi$-squares $P^{\prime} \in \mathcal{T}_{\nu}\left(R^{\prime}\right)$ intersecting $Q^{\prime}$ are contained in $3 Q^{\prime}$. Thus, there are at most four dyadic $\phi$-squares $L_{1}^{\prime}, \ldots, L_{4}^{\prime}$ with $\ell\left(L_{i}\right) \leq 2 \ell(3 Q)$ that contain all $\phi$-squares $P^{\prime} \in \mathcal{T}_{\nu}\left(R^{\prime}\right)$ intersecting $Q^{\prime}$. Then, by construction we have

$$
\nu\left(Q^{\prime}\right) \leq \sum_{i=1}^{4} \tau\left(L_{i}^{\prime}\right) \leq \sum_{i=1}^{4} C_{23} b \eta^{\alpha-\frac{2}{K+1}} M^{h}\left(L_{i}^{\prime} \cap G_{j}^{\prime}\right) \leq C b \eta^{\alpha-\frac{2}{K+1}} h\left(Q^{\prime}\right) .
$$

From (6.14) and the preceding inequality, one easily deduces (6.10) and (6.11).

To prove (6.12), it is enough to show that for each $P^{\prime} \in \mathcal{T}_{\nu}\left(R^{\prime}\right)$

$$
\sum_{Q^{\prime} \in \phi \mathcal{D}: P^{\prime} \subset Q^{\prime} \subset R^{\prime}}\left(\frac{\nu\left(3 Q^{\prime}\right)}{\ell\left(Q^{\prime}\right)^{\frac{2}{K+1}}}\right)^{\frac{K+1}{K}} \leq C b^{\frac{K+1}{K}} .
$$

Suppose that $P^{\prime} \subset S_{j}^{\prime}$. Then we split the sum above as follows:

$$
\sum_{Q^{\prime} \in \phi \mathcal{D}: P^{\prime} \subset Q^{\prime} \subset R^{\prime}}\left(\frac{\nu\left(3 Q^{\prime}\right)}{\ell\left(Q^{\prime}\right)^{\frac{2}{K+1}}}\right)^{\frac{K+1}{K}}=\sum_{Q^{\prime} \in \phi \mathcal{D}: S_{j}^{\prime} \subsetneq Q^{\prime} \subset R^{\prime}} \cdots+\sum_{Q^{\prime} \in \phi \mathcal{D}: P^{\prime} \subset Q^{\prime} \subset S_{j}^{\prime}} \cdots=: T_{1}+T_{2} .
$$

To estimate the first sum recall that by (6.13) we have

$$
\frac{\nu\left(3 Q^{\prime}\right)}{\ell\left(Q^{\prime}\right)^{\frac{2}{K+1}}} \leq C b\left(\frac{\ell\left(Q^{\prime}\right)}{\ell\left(R^{\prime}\right)}\right)^{\alpha-\frac{2}{K+1}} \varepsilon\left(R^{\prime}\right) .
$$

Then it follows that $T_{1} \leq C\left(b \varepsilon\left(R^{\prime}\right)\right)^{\frac{K+1}{K}}$. Recalling that $\varepsilon\left(R^{\prime}\right)=\varepsilon_{a}(R)^{\frac{2 K}{K+1}} \leq C$, we infer that

$$
T_{1} \leq C b^{\frac{K+1}{K}} .
$$


To estimate $T_{2}$ we use that

$$
\frac{\nu\left(3 Q^{\prime}\right)}{\ell\left(Q^{\prime}\right)^{\frac{2}{K+1}}} \leq C b \varepsilon\left(Q^{\prime}\right)
$$

and the fact that $D_{\mu}\left(P, S_{j}\right) \leq C \varepsilon_{a}\left(S_{j}\right)^{2} \leq C \varepsilon_{a}(R)^{2}$, by construction, and so

$$
\sum_{Q^{\prime} \in \phi \mathcal{D}: P^{\prime} \subset Q^{\prime} \subset S_{j}^{\prime}} \varepsilon\left(Q^{\prime}\right)^{\frac{K+1}{K}} \approx D_{\mu}\left(P, S_{j}\right) \leq C \varepsilon\left(R^{\prime}\right)^{\frac{K+1}{K}} \leq C,
$$

and then we deduce that $T_{2} \leq C b^{\frac{K+1}{K}}$.

6.5. Estimates for the Wolff potential of $\nu$ on trees of type S. Recall Definition 5.9 of $\operatorname{Tree}(R)$ for $R \in \operatorname{Sel}_{S}(\mu)$. We denote $\operatorname{Tree}_{\nu}\left(R^{\prime}\right)=\phi(\operatorname{Tree}(R))$.

Lemma 6.4. Let $R^{\prime} \in \operatorname{Sel}_{S}(\nu)$. If $\nu\left(R^{\prime}\right) \leq b h\left(R^{\prime}\right)$, then

$$
\nu\left(Q^{\prime}\right) \leq C_{24} b h\left(Q^{\prime}\right) \quad \text { for all } Q^{\prime} \in \operatorname{Tree}_{\nu}\left(R^{\prime}\right)
$$

and, for each $P^{\prime} \in \mathcal{T}_{\nu}\left(R^{\prime}\right) \cup F_{\nu}\left(R^{\prime}\right)$,

$$
\sum_{Q^{\prime} \in \phi \mathcal{D}: P^{\prime} \subset Q^{\prime} \subset R^{\prime}}\left(\frac{\nu\left(3 Q^{\prime}\right)}{\ell\left(Q^{\prime}\right)^{\frac{2}{K+1}}}\right)^{\frac{K+1}{K}} \leq C(\eta) b^{\frac{K+1}{K}} .
$$

The constant $C_{24}$ above is independent of $\eta$ in the definition of "long trees".

Proof. The arguments to prove (6.16) are very similar to the ones used in Lemma 6.3 to show that analogous estimates hold for the squares contained in the squares $S_{j}$, taking into account that $\mu\left(\bigcup_{P \in \mathcal{T}_{1}(R)} P\right) \approx \mu(R)$, by (6.9). So we skip the details.

On the other hand, from (6.16) we also infer that

$$
\nu\left(3 Q^{\prime}\right) \leq C b h\left(Q^{\prime}\right) \quad \text { for all } Q^{\prime} \in \operatorname{Tree}_{\nu}\left(R^{\prime}\right) .
$$

Then, (6.17) follows from this estimate and the fact that for every $P^{\prime} \in \mathcal{T}_{\nu}\left(R^{\prime}\right)$,

$$
\sum_{Q^{\prime} \in \phi \mathcal{D}: P^{\prime} \subset Q^{\prime} \subset R^{\prime}} \varepsilon\left(Q^{\prime}\right)^{\frac{K+1}{K}} \leq C(\eta),
$$

by (6.9). 


\section{Proof of Theorem 1.1}

Recall that the measure $\nu$ supported on $\phi(E)$ that we have constructed in Section 6 satisfies

$$
\nu(\phi(E))=M^{h}(\phi(E)) \gtrsim \mu(E)^{\frac{2 K}{K+1}} \approx \gamma(E)^{\frac{2 K}{K+1}} .
$$

Thus the theorem follows if we show that

$$
\sum_{Q^{\prime} \in \phi \mathcal{D}: x \in Q^{\prime}}\left(\frac{\nu\left(3 Q^{\prime}\right)}{\ell\left(Q^{\prime}\right)^{\frac{2}{K+1}}}\right)^{\frac{K+1}{K}} \leq C \quad \text { for all } x \in \operatorname{supp}(\nu)
$$

Let $\left\{R_{n}^{\prime}\right\}_{n \geq 0}$ be the collection of $\phi$-squares from $\operatorname{Sel}(\nu)$ which contain $x$. We assume that $\ell\left(R_{n}\right)>\ell\left(R_{n+1}\right)$ for all $n$. We split the preceding sum as follows:

$$
\begin{aligned}
\sum_{Q^{\prime} \in \phi \mathcal{D}: x \in Q^{\prime}}\left(\frac{\nu\left(3 Q^{\prime}\right)}{\ell\left(Q^{\prime}\right)^{\frac{2}{K+1}}}\right)^{\frac{K+1}{K}}= & \sum_{Q^{\prime} \in \phi \mathcal{D}: R_{0}^{\prime} \subsetneq Q^{\prime}}\left(\frac{\nu\left(3 Q^{\prime}\right)}{\ell\left(Q^{\prime}\right)^{\frac{2}{K+1}}}\right)^{\frac{K+1}{K}} \\
& +\sum_{n \geq 0} \sum_{Q^{\prime} \in \phi \mathcal{D}: R_{n+1}^{\prime} \subsetneq Q^{\prime} \subset R_{n}^{\prime}}\left(\frac{\nu\left(3 Q^{\prime}\right)}{\ell\left(Q^{\prime}\right)^{\frac{2}{K+1}}}\right)^{\frac{K+1}{K}}=: S_{1}+S_{2} .
\end{aligned}
$$

To estimate the sum $S_{1}$ on the right side, one only needs to take into account that

$$
\left(\frac{\nu(\phi(E))}{\ell\left(Q^{\prime}\right)^{\frac{2}{K+1}}}\right)^{\frac{K+1}{K}}=\frac{\ell\left(R_{0}^{\prime}\right)^{\frac{2}{K}}}{\ell\left(Q^{\prime}\right)^{\frac{2}{K}}}\left(\frac{\nu(\phi(E))}{\ell\left(R_{0}^{\prime}\right)^{\frac{2}{K+1}}}\right)^{\frac{K+1}{K}} \leq \frac{\ell\left(R_{0}^{\prime}\right)^{\frac{2}{K}}}{\ell\left(Q^{\prime}\right)^{\frac{2}{K}}} \varepsilon\left(R_{0}^{\prime}\right)^{\frac{K+1}{K}} \leq C \frac{\ell\left(R_{0}^{\prime}\right)^{\frac{2}{K}}}{\ell\left(Q^{\prime}\right)^{\frac{2}{K}}}
$$

and summing over those $Q^{\prime} \in \phi \mathcal{D}$ containing $R_{0}^{\prime}$, we get $S_{1} \leq C$.

To deal with $S_{2}$, observe that, by Lemmas 6.3 and 6.4 ,

$$
\sum_{n \geq 0} \sum_{Q^{\prime} \in \phi \mathcal{D}: R_{n+1}^{\prime} \subsetneq Q^{\prime} \subset R_{n}^{\prime}}\left(\frac{\nu\left(3 Q^{\prime}\right)}{\ell\left(Q^{\prime}\right)^{\frac{2}{K+1}}}\right)^{\frac{K+1}{K}} \leq C(\eta) \sum_{n \geq 0}\left(\frac{\nu\left(R_{n}^{\prime}\right)}{h\left(R_{n}^{\prime}\right)}\right)^{\frac{K+1}{K}} .
$$

Lemma 6.3 tells us that if $R_{n}^{\prime} \in \operatorname{Sel}_{L}(\nu)$, then

$$
\frac{\nu\left(R_{n+1}^{\prime}\right)}{h\left(R_{n+1}^{\prime}\right)} \leq C_{25} \eta^{\alpha-\frac{2}{K+1}} \frac{\nu\left(R_{n}^{\prime}\right)}{h\left(R_{n}^{\prime}\right)}
$$

and if $R_{n}^{\prime} \in \operatorname{Sel}_{S}(\nu)$, then

$$
\frac{\nu\left(R_{n+1}^{\prime}\right)}{h\left(R_{n+1}^{\prime}\right)} \leq C_{25} \frac{\nu\left(R_{n}^{\prime}\right)}{h\left(R_{n}^{\prime}\right)}
$$

where $C_{25}$ is the maximum of the corresponding constants $C_{22}$ and $C_{24}$ in (6.11) and (6.16). Notice that, by construction, for all $m$, it turns out that either $R_{m}^{\prime}$ or $R_{m+1}^{\prime}$ 
belongs to $\operatorname{Sel}_{L}(\nu)$. As a consequence,

$$
\sum_{n \geq 0}\left(\frac{\nu\left(R_{n}^{\prime}\right)}{h\left(R_{n}^{\prime}\right)}\right)^{\frac{K+1}{K}} \leq \sum_{n \geq 0}\left(C_{25} \eta^{\frac{1}{2}\left(\alpha-\frac{2}{K+1}\right)}\right)^{\frac{K+1}{K} n} \leq C
$$

if $\eta$ is chosen small enough (recall that $C_{25}$ is independent of $\eta$ ). Thus, $S_{2} \leq C(\eta)$ and (7.1) follows.

\section{EXAMPLES SHOWING SHARPNESS OF RESULTS}

8.1. Some results from $\left[\mathrm{ACM}^{+} 08\right]$. The state-of-the-art for largest "metric" (or "size") sufficient conditions for removability theorems for bounded $K$ quasiregular maps is given by Theorem 1.2 in $\left[\mathrm{ACM}^{+} 08\right]$.

Theorem 8.1 (Astala, Clop, Mateu, Orobitg, Uriarte-Tuero). Let $K>1$ and suppose $E \subset \mathbb{C}$ is a compact set with $\mathcal{H}^{\frac{2}{K+1}}(E) \sigma$-finite. Then $E$ is removable for bounded $K$ quasiregular maps.

As a first remark, let us mention that from Theorem 1.1 we recover this result. Indeed, if $E \subset \mathbb{C}$ and $\mathcal{H}^{\frac{2}{K+1}}(E)<\infty$, then $\dot{C}_{\frac{2 K}{2 K+1}}, \frac{2 K+1}{K+1}(E)=0$. Also if $E_{i} \subset \mathbb{C}$, for $i=1,2, \ldots$ and $E=\bigcup_{i=1}^{\infty} E_{i}$ with $\mathcal{H}^{\frac{2}{K+1}}\left(E_{i}\right)<\infty$, then $\dot{C}_{\frac{2 K}{2 K+1}, \frac{2 K+1}{K+1}}(E) \leq$ $\sum_{i=1}^{\infty} \dot{C}_{\frac{2 K}{2 K+1}}, \frac{2 K+1}{K+1}\left(E_{i}\right)=0$, by the subadditivity of $\dot{C}_{\frac{2 K}{2 K+1}, \frac{2 K+1}{K+1}}$ (see AH96, Proposition 2.3.6], for example). Consequently, recalling that by Stoilow's factorization any $K$-quasiregular map $f$ can be factored as $f=h \circ g$, where $h$ is analytic and $g$ is $K$-quasiconformal, by Theorem 1.1 in the present paper, $E$ is removable.

Of course, in order to prove Theorem 1.1, we used many of the ideas in $\mathrm{ACM}^{+} 08$, so we are not claiming any novelty.

To contextualize some of our examples below, we recall the next result from $\left.\mathrm{ACM}^{+} 08\right]$.

Theorem 8.2 (Astala, Clop, Mateu, Orobitg, Uriarte-Tuero). Let $K \geq 1$. Suppose $h(t)=t^{\frac{2}{K+1}} \varepsilon(t)$ is a measure function such that

$$
\int_{0} \frac{\varepsilon(t)^{1+1 / K}}{t} d t<\infty
$$

Then there is a compact set $E$ which is not $K$-removable and such that $0<\mathcal{H}^{h}(E)<$ $\infty$. In particular, whenever $\varepsilon(t)$ is chosen so that in addition for every $\alpha>0$ we have $t^{\alpha} / \varepsilon(t) \rightarrow 0$ as $t \rightarrow 0$, then the construction gives a non-K-removable set $E$ with $\operatorname{dim}(E)=\frac{2}{K+1}$. 
8.2. Example 1. Our next example shows that Theorem 1.1 is strictly stronger than Theorem 8.1. Indeed, let us recall Theorem 5.4.2 in [AH96], adapted to our situation.

Theorem 8.3. Let $h$ be an increasing nonnegative function on $[0, \infty)$. If

$$
\int_{0}^{1}\left(\frac{h(r)}{r^{\frac{2}{K+1}}}\right)^{1+\frac{1}{K}} \frac{d r}{r}=\infty,
$$

then there is a compact set $E \subset \mathbb{C}$ such that $\mathcal{H}^{h}(E)>0$ and $\dot{C}_{\frac{2 K}{2 K+1}}, \frac{2 K+1}{K+1}(E)=0$.

If we choose $h(r)$ so that it satisfies the conditions in Theorem 8.3 but $\frac{h(r)}{r^{\frac{2}{K+1}}} \rightarrow 0$ as $t \rightarrow 0$, then the set $E$ obtained in Theorem 8.3 will be non- $\sigma$-finite for $\mathcal{H}^{\frac{2}{K+1}}$, but will be removable for bounded $K$-quasiregular maps due to Theorem 1.1 and Stoilow's factorization. For this purpose it is enough to choose $h(r)=\frac{r^{\frac{2}{K+1}}}{\log \left(\frac{1}{r}\right)^{\beta}}$ when $r$ is small enough, so that $\beta>0$ and $\beta\left(1+\frac{1}{K}\right) \leq 1$.

8.3. Basic construction for the subsequent examples. For our subsequent examples we need to refine the construction from Theorem 8.2. To this end we argue as in [UT08]. We assume the reader is familiar with that paper and we will use the notation from it without further reference. The formulae look slightly nicer if we assume in the construction that $\varepsilon_{n}=0$ for all $n$, i.e. that we take infinitely many disks in each step, completely filling the area of the unit disk $\mathbb{D}$ (see equations (2.1), (2.2) and (2.3) in [UT08].) It is not strictly needed to set in that construction $\varepsilon_{n}=0$ for all $n$, and we will later indicate the corresponding formulae if $\varepsilon_{n}>0$ for all $n$ (which is the case in [UT08.) The construction in [UT08] works as well if we set $\varepsilon_{n}=0$ for all $n$, the only point that the reader might wonder about is whether the resulting map is $K$-quasiconformal. However, this can be seen easily by a compactness argument (approximating the desired map by maps with finitely many circles in each step which are $K$-quasiconformal and have more and more disks in each step of the construction).

So we get (see equations (2.5) and (2.6) in [UT08) a Cantor type set $E$ and a $K$-quasiconformal map $\phi$ so that a building block in the $N^{\text {th }}$ step of the construction of the source set $E$ is a disk with radius given by

$$
s_{j_{1}, \ldots, j_{N}}=\left(\left(\sigma_{1, j_{1}}\right)^{K} R_{1, j_{1}}\right) \ldots\left(\left(\sigma_{N, j_{N}}\right)^{K} R_{N, j_{N}}\right),
$$

and a building block in the $N^{\text {th }}$ step of the construction in the target set $\phi(E)$ is a disk with radius given by

$$
t_{j_{1}, \ldots, j_{N}}=\left(\sigma_{1, j_{1}} R_{1, j_{1}}\right) \ldots\left(\sigma_{N, j_{N}} R_{N, j_{N}}\right) .
$$

Now we consider a measure $\mu$ supported on $\phi(E)$ (which will be the "large" set of dimension $d^{\prime}=1$ ) and its image measure $\nu=\phi_{*}^{-1} \mu$ supported on $E$ (which will be the "small" set of dimension $d=\frac{2}{K+1}$ ) given by splitting the mass according to area. More explicitly, 


$$
\mu(\mathbb{D})=1
$$

for any disk $B_{1, j_{1}}=\psi_{1, j_{1}}^{i_{1}}(\overline{\mathbb{D}})$ of the first step of the construction with radius $t_{j_{1}}=\left(\sigma_{1, j_{1}} R_{1, j_{1}}\right)$

$$
\mu\left(B_{1, j_{1}}\right)=\left(R_{1, j_{1}}\right)^{2}
$$

and in general, for any disk $B_{N ; j_{1}, \ldots, j_{N}}^{i_{1}, \ldots, i_{N}}=\psi_{1, j_{1}}^{i_{1}} \circ \cdots \circ \psi_{N, j_{N}}^{i_{N}}(\overline{\mathbb{D}})$ of the $N^{\text {th }}$ step of the construction with radius $t_{j_{1}, \ldots, j_{N}}=\left(\sigma_{1, j_{1}} R_{1, j_{1}}\right) \ldots\left(\sigma_{N, j_{N}} R_{N, j_{N}}\right)$,

$$
\mu\left(B_{N ; j_{1}, \ldots, j_{N}}^{i_{1}, \ldots, i_{N}}\right)=\left(R_{1, j_{1}} \ldots R_{N, j_{N}}\right)^{2}
$$

Since we took $\varepsilon_{N}=0$ for all $N$, the total mass of $\mu$ is always 1 on every step. (If one prefers to take $\varepsilon_{N}>0$ for all $N$, the definition should be changed to $\mu\left(B_{N ; j_{1}, \ldots, j_{N}}^{i_{1}, \ldots, i_{N}}\right)=$ $\left(R_{1, j_{1}} \ldots R_{N, j_{N}}\right)^{2} \quad \prod_{n=N+1}^{\infty}\left(1-\varepsilon_{n}\right)$, and the total mass of $\mu$ gets renormalized by the factor $\prod_{n=1}^{\infty}\left(1-\varepsilon_{n}\right)>0$, but otherwise the rest of the construction we are about to describe works, keeping in mind these renormalizations.)

Since $\nu$ is the image measure, for any disk $D_{N ; j_{1}, \ldots, j_{N}}^{i_{1}, \ldots, i_{N}}=\varphi_{1, j_{1}}^{i_{1}} \circ \cdots \circ \varphi_{N, j_{N}}^{i_{N}}(\overline{\mathbb{D}})=$ $\phi^{-1}\left(B_{N ; j_{1}, \ldots, j_{N}}^{i_{1}, \ldots, i_{N}}=\varphi_{1, j_{1}}^{i_{1}} \circ \cdots \circ \varphi_{N, j_{N}}^{i_{N}}(\overline{\mathbb{D}})\right)$ we get

$$
\nu\left(D_{N ; j_{1}, \ldots, j_{N}}^{i_{1}, \ldots, i_{N}}\right)=\left(R_{1, j_{1}} \ldots R_{N, j_{N}}\right)^{2} .
$$

Lemma 8.4. For the Cantor type sets just described (in subsection 8.3), for any $\alpha, p>0$ with $\alpha p<2$, and for $x \in E$, the Wolff potentials satisfy

$$
\dot{W}_{\alpha, p}^{\mu}(x) \approx \sum_{n}\left(\frac{\mu\left(B\left(x, 2^{n}\right)\right)}{2^{n(2-\alpha p)}}\right)^{p^{\prime}-1} \approx \sum_{N: x \in B_{N ; j_{1}, \ldots, j_{N}}^{i_{1}, \ldots, i_{N}}}\left(\frac{\mu\left(B_{N ; j_{1}, \ldots, j_{N}}^{i_{1}, \ldots, i_{N}}\right)}{\left(t_{j_{1}, \ldots, j_{N}}\right)^{(2-\alpha p)}}\right)^{p^{\prime}-1}
$$

and analogously for $\nu, D_{N ; j_{1}, \ldots, j_{N}}^{i_{1}, \ldots, i_{N}}$ and $s_{j_{1}, \ldots, j_{N}}$.

Proof. We first introduce some convenient notation. For any multiindexes $I=$ $\left(i_{1}, \ldots, i_{N}\right)$ and $J=\left(j_{1}, \ldots, j_{N}\right)$, where $1 \leq i_{k}, j_{k} \leq \infty$ (since we are taking infinitely many disks in each step of the construction), we will denote by

$$
P_{I ; J}^{N}=\frac{1}{\sigma_{N, j_{N}}} \psi_{1, j_{1}}^{i_{1}} \circ \cdots \circ \psi_{N, j_{N}}^{i_{N}}(\mathbb{D})
$$

a protecting disk of generation $N$. Then, $P_{I ; J}^{N}$ has radius

$$
r\left(P_{I ; J}^{N}\right)=\frac{1}{\sigma_{N, j_{N}}} t_{j_{1}, \ldots, j_{N}}=\left(\sigma_{1, j_{1}} \ldots \sigma_{N-1, j_{N-1}}\right)\left(R_{1, j_{1}} \ldots R_{N, j_{N}}\right) .
$$

Analogously, we will write

$$
G_{I ; J}^{N}=\psi_{1, j_{1}}^{i_{1}} \circ \cdots \circ \psi_{N, j_{N}}^{i_{N}}(\mathbb{D})
$$

in order to denote a generating disk of generation $N$, which has radius

$$
r\left(G_{I ; J}^{N}\right)=t_{j_{1}, \ldots, j_{N}}=\left(\sigma_{1, j_{1}} \ldots \sigma_{N, j_{N}}\right)\left(R_{1, j_{1}} \ldots R_{N, j_{N}}\right) .
$$


Notice that, since all values of $\sigma_{n, j_{n}}$ and $R_{n, j_{n}}$ are $\leq \frac{1}{100}$, then $\mu\left(G_{I ; J}^{N}\right)=\mu\left(2 G_{I ; J}^{N}\right)$, so we can pretend without loss of generality that the radii $t_{j_{1}, \ldots, j_{N}}$ are dyadic numbers.

Now, if $r\left(G_{I ; J}^{N}\right) \lesssim t \lesssim r\left(P_{I ; J}^{N}\right)$, and $x \in E$ so that $B(x, t) \subseteq P_{I ; J}^{N}$, then $\mu(B(x, t))=$ $\mu\left(G_{I ; J}^{N}\right)$, so that

$$
\sum_{n: G_{I ; J}^{N} \subseteq B\left(x, 2^{n}\right) \subseteq P_{I ; J}^{N}}\left(\frac{\mu\left(B\left(x, 2^{n}\right)\right)}{2^{n(2-\alpha p)}}\right)^{p^{\prime}-1}
$$

is a geometric series with sum comparable (with constants of comparison only depending on $\alpha$ and $p$ ) to its largest term, namely, up to universal constants, $\left(\frac{\mu\left(G_{I ; J}^{N}\right)}{\left(t_{j_{1}, \ldots, j_{N}}\right)^{(2-\alpha p)}}\right)^{p^{\prime}-1}$.

And if $r\left(P_{I ; J}^{N}\right) \lesssim t \lesssim r\left(G_{I^{\prime} ; J^{\prime}}^{N-1}\right)$, where $G_{I^{\prime} ; J^{\prime}}^{N-1}$ is the unique generating disk of generation $N-1$ containing $P_{I ; J}^{N}$, and $x \in E$ so that $P_{I ; J}^{N} \subseteq B(x, t) \subseteq G_{I^{\prime} ; J^{\prime}}^{N-1}$, then

$$
\mu(B(x, t)) \lesssim \frac{t^{2}}{\left(\sigma_{1, j_{1}} \ldots \sigma_{N-1, j_{N-1}}\right)\left(R_{1, j_{1}} \ldots R_{N-1, j_{N-1}}\right)}\left(R_{1, j_{1}} \ldots R_{N-1, j_{N-1}}\right)^{2}
$$

i.e. the mass that $\mu$ assigns to $B(x, t)$ is proportional to its area once $G_{I^{\prime} ; J^{\prime}}^{N-1}$ is renormalized to $\mathbb{D}$, but multiplied by the mass that $\mu$ assigns to $G_{I^{\prime} ; J^{\prime}}^{N-1}$, namely $\left(R_{1, j_{1}} \ldots R_{N-1, j_{N-1}}\right)^{2}$. Hence

$$
\sum_{n: P_{I ; J}^{N} \subseteq B\left(x, 2^{n}\right) \subseteq G_{I^{\prime} ; J^{\prime}}^{N-1}}\left(\frac{\mu\left(B\left(x, 2^{n}\right)\right)}{2^{n(2-\alpha p)}}\right)^{p^{\prime}-1}
$$

is dominated by a geometric series (if $n$ appears in the above sum and $2^{n}=\frac{r\left(G_{I^{\prime} ; J^{\prime}}^{N-1}\right)}{2^{k}}$ with $k>0$, then $\left(\frac{\mu\left(B\left(x, 2^{n}\right)\right)}{2^{n(2-\alpha p)}}\right)^{p^{\prime}-1} \lesssim\left(\frac{\mu\left(G_{I^{\prime} ; J^{\prime}}^{N-1}\right)}{r\left(G_{I^{\prime} ; J^{\prime}}^{N}\right)^{(2-\alpha p)}} \frac{2^{k(2-\alpha p)}}{2^{2 k}}\right)^{p^{\prime}-1}$, and hence the above sum is $\lesssim\left(\frac{\mu\left(G_{I^{\prime} ; J^{\prime}}^{N-1}\right)}{r\left(G_{I^{\prime} ; J^{\prime}}^{\prime-1}\right)^{(2-\alpha p)}}\right)^{p^{\prime}-1}$, with constants of comparison only depending on $\alpha$ and $p$.)

8.4. Example 2. In view of example 1, it is natural to wonder whether all compact sets $E$ with $\mathcal{H}^{h}(E)=0$ for some gauge function $h(r)$ satisfying $\frac{h(r)}{r^{\frac{2}{K+1}}} \rightarrow 0$ are removable for bounded $K$-quasiregular maps, i.e. whether there is some condition strictly weaker than $\mathcal{H}^{\frac{2}{K+1}}(E)$ being $\sigma$-finite in terms of the gauge function $h$, which guarantees removability. Our next example shows that this is not the case. Notice the resemblance to Theorem 5.4.1 in [AH96].

Theorem 8.5. Let $h$ be a positive function on $(0, \infty)$ such that

$$
\varepsilon(r)=\frac{h(r)}{r^{\frac{2}{K+1}}} \rightarrow 0 \quad \text { as } r \rightarrow 0 .
$$


Then there is a compact set $E \subset \mathbb{C}$ such that $\mathcal{H}^{h}(E)=0$ and a $K$-quasiconformal map $\phi$ such that $\gamma(\phi E)>0$ (and hence $\dot{C}_{\frac{2 K}{2 K+1}}, \frac{2 K+1}{K+1}(E)>0$, due to Theorem 1.1.)

Proof. For $E$ and $\phi$ as in Subsection 8.3, notice that by Lemma 8.4, for $x \in \phi E$

$$
\dot{W}_{\frac{2}{3}, \frac{3}{2}}^{\mu}(x) \approx \sum_{N: x \in B_{N ; j_{1}, \ldots, j_{N}}^{i_{1}, \ldots, i_{N}}}\left(\frac{\mu\left(B_{N ; j_{1}, \ldots, j_{N}}^{i_{1}, \ldots, i_{N}}\right)}{\left(t_{j_{1}, \ldots, j_{N}}\right)}\right)^{2}=\sum_{N: x \in B_{N ; j_{1}, \ldots, j_{N}}^{i_{1}, \ldots, i_{N}}}\left(\frac{R_{1, j_{1}} \ldots R_{N, j_{N}}}{\sigma_{1, j_{1}} \ldots \sigma_{N, j_{N}}}\right)^{2}
$$

Since on the one hand $E$ is very "close" to satisfying $0<\mathcal{H}^{\frac{2}{K+1}}(E)<\infty$ and $0<\mathcal{H}^{1}(\phi E)<\infty$ (see (3.11) and (4.5) in [UT08]), and on the other hand an important element in the proof of the semiadditivity of analytic capacity is that the potential is "approximately constant" on each scale (see [Tol03]), the above equation suggests the choice

$$
\sigma_{N, j_{N}}=R_{N, j_{N}} d_{N} \quad \text { for all } N
$$

where $d_{N} \in[1,2]$ is a parameter to be determined, independent of $j_{N}$.

If we take

$$
d_{j}=\frac{j+1}{j}
$$

then, for $x \in \phi E$, we have

$$
\dot{W}_{\frac{2}{3}, \frac{3}{2}}^{\mu}(x) \approx \sum_{n}\left\{\prod_{j=1}^{n} \frac{1}{\left(d_{j}\right)^{2}}\right\}=\sum_{n=2}^{\infty} \frac{1}{n^{2}}<\infty
$$

so that $\dot{C}_{\frac{2}{3}, \frac{3}{2}}(\phi E)>0$, and $\gamma(\phi E)>0$.

Let us denote $\varepsilon_{\max }^{N}=\max \left\{\varepsilon\left(s_{j_{1}, \ldots, j_{N}}\right)\right\}$. For each $N$, substituting $\sigma_{N, j_{N}}=$ $R_{N, j_{N}} d_{N}$, recalling that $\sum_{j_{1}, \ldots, j_{N}}\left(R_{1, j_{1}} \ldots R_{N, j_{N}}\right)^{2}=1$, and that $d_{n}=\frac{n+1}{n}$, we obtain

$$
\begin{aligned}
\sum_{j_{1}, \ldots, j_{N}} h\left(r\left(D_{N ; j_{1}, \ldots, j_{N}}^{i_{1}, \ldots, i_{N}}\right)\right) & =\sum_{j_{1}, \ldots, j_{N}} h\left(s_{j_{1}, \ldots, j_{N}}\right) \\
& =\sum_{j_{1}, \ldots, j_{N}} \varepsilon\left(s_{j_{1}, \ldots, j_{N}}\right)\left(\left(\sigma_{1, j_{1}}\right)^{K} R_{1, j_{1}}\right) \ldots\left(\left(\sigma_{N, j_{N}}\right)^{K} R_{N, j_{N}}\right)^{\frac{2}{K+1}} \\
& \leq \varepsilon_{\max }^{N}\left(d_{1} \ldots d_{N}\right)^{\frac{2 K}{K+1}} \sum_{j_{1}, \ldots, j_{N}}\left(R_{1, j_{1}} \ldots R_{N, j_{N}}\right)^{2} \\
& =\varepsilon_{\max }^{N}\left(d_{1} \ldots d_{N}\right)^{\frac{2 K}{K+1}}=\varepsilon_{\max }^{N}(N+1)^{\frac{2 K}{K+1}}
\end{aligned}
$$

Choosing $R_{1, j_{1}} \ldots R_{N, j_{N}}$ small enough in the construction so that $\varepsilon_{\max }^{N}(N+1)^{\frac{2 K}{K+1}} \rightarrow$ 0 as $N \rightarrow \infty$, one infers that $\mathcal{H}^{h}(E)=0$. 
8.5. Example 3. The preceding example can be modified (notice the analogies with Theorem 5.6.4 in [AH96]) to show that

Theorem 8.6. There is a compact set $E \subset \mathbb{C}$ such that $\gamma(\phi E)>0$ (and hence $\dot{C}_{\frac{2 K}{2 K+1}, \frac{2 K+1}{K+1}}(E)>0$, due to Theorem 1.1), but $\mathcal{H}^{h}(E)=0$ for every positive function $h$ such that

and

$$
\varepsilon(r)=\frac{h(r)}{r^{\frac{2}{K+1}}} \text { is non decreasing, }
$$

$$
\int_{0}^{1}\left(\frac{h(r)}{r^{\frac{2}{K+1}}}\right)^{a} \frac{d r}{r}<\infty, \text { for some } a>0
$$

Proof. In the preceding construction, denote $S_{\max }^{N}=\max \left\{s_{j_{1}, \ldots, j_{N}}\right\}$ and choose $S_{\max }^{N} \leq e^{-e^{N}}$. Since $\varepsilon$ is non decreasing, $\varepsilon\left(s_{j_{1}, \ldots, j_{N}}\right) \leq \varepsilon\left(e^{-e^{N}}\right)$, and from (8.13) we deduce

$$
\left[\mathcal{H}^{h}(E)\right]^{a} \lesssim \liminf _{N \rightarrow \infty}\left\{\left[\varepsilon\left(S_{\max }^{N}\right)\right]^{a} N^{\frac{2 K a}{K+1}}\right\} \lesssim \liminf _{N \rightarrow \infty}\left\{\sum_{n=N}^{\infty}\left[\varepsilon\left(e^{-e^{n}}\right)\right]^{a} n^{\frac{2 K a}{K+1}}\right\} .
$$

Using that again that $\varepsilon$ is non decreasing and setting $s=e^{-\frac{1}{t}}$, we obtain

$$
\begin{aligned}
{\left[\mathcal{H}^{h}(E)\right]^{a} } & \lesssim \liminf _{N \rightarrow \infty}\left\{\sum_{n=N}^{\infty} \int_{e^{-n}}^{e^{-n+1}}\left[\varepsilon\left(e^{-e^{n}}\right)\right]^{a}\left[\log \left(\frac{1}{t}\right)\right]^{\frac{2 K a}{K+1}} \frac{d t}{t}\right\} \\
& \leq \liminf _{N \rightarrow \infty}\left\{\int_{0}^{e^{-N+1}}\left[\varepsilon\left(e^{-\frac{1}{t}}\right)\right]^{a}\left[\log \left(\frac{1}{t}\right)\right]^{\frac{2 K a}{K+1}} \frac{d t}{t}\right\} \\
& =\liminf _{N \rightarrow \infty}\left\{\int_{0}^{e^{-e^{N-1}}}[\varepsilon(s)]^{a} \frac{\left[\log \log \left(\frac{1}{s}\right)\right]^{\frac{2 K a}{K+1}}}{\log \left(\frac{1}{s}\right)} \frac{d s}{s}\right\} \\
& \lesssim \liminf _{N \rightarrow \infty}\left\{\int_{0}^{e^{-e^{N-1}}}[\varepsilon(s)]^{a} \frac{d s}{s}\right\}=0 .
\end{aligned}
$$

8.6. Example 4. Examples 2 and 3 strongly suggest that the language of capacities $\dot{C}_{\alpha, p}$ is better suited to understand the removability for bounded $K$-quasiregular maps than the language of Hausdorff measures. Hence it is natural to wonder how sharp Theorem 1.1 is in the category of capacities $\dot{C}_{\alpha, p}$. To that effect, it is useful to recall Theorem 5.5.1 (b) in [AH96] adapted to our situation (and combined with Proposition 5.1.4):

Theorem 8.7. Let $E \subset \mathbb{C}$. Then there is a constant $A$ such that

$$
\dot{C}_{\beta, q}(E) \leq A \dot{C}_{\alpha, p}(E)
$$

for $\beta q=\alpha p=2-\frac{2}{K+1}=\frac{2 K}{K+1}, p<q$. 
Moreover, there exist sets $E$ such that $\dot{C}_{\beta, q}(E)=0$ but $\dot{C}_{\alpha, p}(E)>0$.

Hence it is conceivable that Theorem 1.1 might be strengthened to a statement of the form

$$
\frac{\dot{C}_{\beta, q}(E)}{\operatorname{diam}(B)^{\frac{2}{K+1}}} \geq c^{-1}\left(\frac{\gamma(\phi(E))}{\operatorname{diam}(\phi(B))}\right)^{\frac{2 K}{K+1}}
$$

for some $\beta, q$ such that $\beta q=\frac{2 K}{K+1}$ and $\frac{2 K+1}{K+1}<q$, i.e. for $q^{\prime}-1<1+\frac{1}{K}$. The following theorem shows that the answer to this question is negative.

Theorem 8.8. For any $\beta, q>0$ such that $\beta q=\frac{2 K}{K+1}$ and $\frac{2 K+1}{K+1}<q$, there exists a compact $E \subset \mathbb{C}$ and a $K$-quasiconformal map $\phi$ such that $\gamma(\phi E)>0$ (and hence $\dot{C}_{\frac{2 K}{2 K+1}, \frac{2 K+1}{K+1}}(E)>0$, due to Theorem 1.1), but $\dot{C}_{\beta, q}(E)=0$.

Proof. As in the construction in Example 2, we choose $\sigma_{N, j_{N}}=R_{N, j_{N}} d_{N}$. Then, for $y \in \phi E$,

$$
\dot{W}_{\frac{2}{3}, \frac{3}{2}}^{\mu}(y) \approx \sum_{n}\left\{\prod_{j=1}^{n} \frac{1}{\left(d_{j}\right)^{2}}\right\}
$$

while by Lemma 8.4 and (8.2), for $x \in E$,

$$
\dot{W}_{\beta, q}^{\nu}(x) \approx \sum_{N: x \in D_{N ; j_{1}, \ldots, j_{N}}^{i_{1}, \ldots, i_{N}}}\left(\frac{\nu\left(D_{N ; j_{1}, \ldots, j_{N}}^{i_{1}, \ldots, i_{N}}\right)}{\left(s_{j_{1}, \ldots, j_{N}}\right)^{\frac{2}{K+1}}}\right)^{q^{\prime}-1}=\sum_{N: x \in D_{N ; j_{1}, \ldots, j_{N}}^{i_{1}, \ldots, i_{N}}}\left(\frac{R_{1, j_{1}} \ldots R_{N, j_{N}}}{\sigma_{1, j_{1}} \ldots \sigma_{N, j_{N}}}\right)^{\frac{2 K}{K+1}\left(q^{\prime}-1\right)}
$$

so that, substituting $\sigma_{N, j_{N}}=R_{N, j_{N}} d_{N}$ we get, for $x \in E$,

$$
\dot{W}_{\beta, q}^{\nu}(x) \approx \sum_{n}\left\{\prod_{j=1}^{n} \frac{1}{\left(d_{j}\right)^{2}}\right\}^{\left(q^{\prime}-1\right)\left(\frac{K}{K+1}\right)}
$$

Now choose $\left(d_{j}\right)^{2\left(q^{\prime}-1\right)\left(\frac{K}{K+1}\right)}=\frac{j+1}{j}$, so that for $x \in E, \dot{W}_{\beta, q}^{\nu}(x) \approx \sum_{n=2}^{\infty} \frac{1}{n}=\infty$, while for $y \in \phi E$,

$$
\dot{W}_{\frac{2}{3}, \frac{3}{2}}^{\mu}(y) \approx \sum_{n=2}^{\infty} \frac{1}{\left\{n^{\frac{1}{\left(q^{\prime}-1\right)\left(\frac{K}{K+1}\right)}}\right\}}<\infty
$$

since $\left(q^{\prime}-1\right)\left(\frac{K}{K+1}\right)<1$.

The fact that $\dot{W}_{\frac{2}{3}, \frac{3}{2}}^{\mu}(y)<\infty$ for all $y \in \phi(E)$ implies that $\dot{C}_{\frac{2}{3}, \frac{3}{2}}(\phi E)>0$, and hence $\gamma(\phi E)>0$ and $\left.\dot{C}_{\frac{2 K}{2 K+1}}, \frac{2 K+1}{K+1}(E)>0\right)$, while from the fact that $\dot{W}_{\beta, q}^{\nu}(x)=\infty$ for all $x \in E$ one infers that $\dot{C}_{\beta, q}(E)=0$ (see Proposition 6.3.12 and (6.3.4) in [AH96], adapted for the potential $\dot{W}_{\beta, q}^{\nu}$.) 
Let us remark that the above example also gives that $\dot{C}_{\gamma, r}(E)=0$ if $\gamma r<\beta q=$ $2 K /(K+1)$. This due to the fact that there is some constant $A$ indendent of $E$ such that

$$
\dot{C}_{\gamma, r}(E)^{1 /(2-\gamma r)} \leq A \dot{C}_{\beta, q}(E)^{1 /(2-\beta q)} .
$$

See Theorem 5.5.1 of [AH96].

\section{FinAL REMARKS}

The Main Lemma 2.11 on the distortion of $h$-contents can also be proved using arguments based on the ideas in [LSUT10], instead of [ $\left.\mathrm{ACM}^{+} 08\right]$. Following this new approach one can extend the Main Lemma 2.11 to $h$-contents $M^{h}$, with $h$ of the form $h(B(x, r))=r^{t} \varepsilon(B(x, r))$, for all $0<t<2$. As a consequence, one can extend Theorem 1.2 (a) to all capacities $\dot{C}_{\alpha, p}$, with $\alpha>0,1<p<\infty$, such that $\alpha p<2$. Then, one obtains the following:

Theorem 9.1. Let $1<q<\infty$ and $0<\beta q<2$. Let $t^{\prime}=2-\beta q$, and $t$ be such that

$$
\frac{1}{t}-\frac{1}{2}=K\left(\frac{1}{t^{\prime}}-\frac{1}{2}\right)
$$

Let $E \subset \mathbb{C}$ be compact, and let $\phi: \mathbb{C} \rightarrow \mathbb{C}$ be a $K$-quasiconformal map. If $E$ is contained in a ball $B$, then

$$
\frac{\dot{\mathcal{C}}_{\beta, q}(\phi(E))}{\operatorname{diam}(\phi(B))^{t^{\prime}}} \leq C(\beta, q, K)\left(\frac{\dot{\mathcal{C}}_{\alpha, p}(E)}{\operatorname{diam}(B)^{t}}\right)^{\frac{t^{\prime}}{K t}}
$$

where

$$
p=1+\frac{K t}{t^{\prime}}(q-1) \quad \text { and } \quad 2-\alpha p=t .
$$

The constant in (9.1) depends only on $\beta, q, K$.

The proof will appear in $\left[\mathrm{ACT}^{+}\right]$.

Acknowledgements. The second named author would like to thank K. Astala, J. Mateu, J. Orobitg, and J. Verdera for fruitful conversations regarding some parts this paper.

\section{REFERENCES}

$\left[\mathrm{ACM}^{+} 08\right]$ Kari Astala, Albert Clop, Joan Mateu, Joan Orobitg, and Ignacio Uriarte-Tuero. Distortion of Hausdorff measures and improved Painlevé removability for bounded quasiregular mappings. Duke Math. J., 141(3):539-571, 2008.

$\left[\mathrm{ACT}^{+}\right] \quad$ Kari Astala, Albert Clop, Xavier Tolsa, Ignacio Uriarte-Tuero, and Joan Verdera. Quasiconformal distortion of Riesz capacities and Hausdorff measures in the plane. Preprint, 2010. To appear in American Journal of Mathematics. See http://arxiv.org/pdf/1002.1038.pdf. 
[AH96] David R. Adams and Lars Inge Hedberg. Function spaces and potential theory, volume 314 of Grundlehren der Mathematischen Wissenschaften [Fundamental Principles of Mathematical Sciences]. Springer-Verlag, Berlin, 1996.

[AIM09] Kari Astala, Tadeusz Iwaniec, and Gaven Martin. Elliptic partial differential equations and quasiconformal mappings in the plane, volume 48 of Princeton Mathematical Series. Princeton University Press, Princeton, NJ, 2009.

[AIS01] Kari Astala, Tadeusz Iwaniec, and Eero Saksman. Beltrami operators in the plane. Duke Math. J., 107(1):27-56, 2001.

[AN03] Kari Astala and Vincenzo Nesi. Composites and quasiconformal mappings: new optimal bounds in two dimensions. Calc. Var. Partial Differential Equations, 18(4):335-355, 2003.

[Ast94] Kari Astala. Area distortion of quasiconformal mappings. Acta Math., 173(1):37-60, 1994.

[CT08] Albert Clop and Xavier Tolsa. Analytic capacity and quasiconformal mappings with $W^{1,2}$ Beltrami coefficient. Math. Res. Lett., 15(4):779-793, 2008.

[Dav98] Guy David. Unrectifiable 1-sets have vanishing analytic capacity. Rev. Mat. Iberoamericana, 14(2):369-479, 1998.

[ENV10] V. Eiderman, F. Nazarov, and A. Volberg. Vector-valued Riesz potentials: Cartan-type estimates and related capacities. Proc. Lond. Math. Soc. (3), 101(3):727-758, 2010.

[LSUT10] Michael T. Lacey, Eric T. Sawyer, and Ignacio Uriarte-Tuero. Astala's conjecture on distortion of Hausdorff measures under quasiconformal maps in the plane. Acta Math., 204(2):273-292, 2010.

[Mat95] Pertti Mattila. Geometry of sets and measures in Euclidean spaces, volume 44 of Cambridge Studies in Advanced Mathematics. Cambridge University Press, Cambridge, 1995. Fractals and rectifiability.

[Mat96] Pertti Mattila. On the analytic capacity and curvature of some Cantor sets with non $\sigma$-finite length. Pub. Mat., 40, 1996.

[Mel95] M. S. Mel'nikov. Analytic capacity: a discrete approach and the curvature of measure. Mat. Sb., 186(6):57-76, 1995.

[MPV05] Joan Mateu, Laura Prat, and Joan Verdera. The capacity associated to signed Riesz kernels, and Wolff potentials. J. Reine Angew. Math., 578:201-223, 2005.

[MTV03] Joan Mateu, Xavier Tolsa, and Joan Verdera. The planar Cantor sets of zero analytic capacity and the local T(b)-theorem. J. Amer. Math. Soc., 16(1):19-28 (electronic), 2003.

[Par90] P. V. Paramonov. Harmonic approximations in the $C^{1}$-norm. Mat. Sb., 181(10):1341$1365,1990$.

[Pom92] Ch. Pommerenke. Boundary behaviour of conformal maps, volume 299 of Grundlehren der Mathematischen Wissenschaften [Fundamental Principles of Mathematical Sciences]. Springer-Verlag, Berlin, 1992.

[Pra04] Laura Prat. Potential theory of signed Riesz kernels: capacity and Hausdorff measure. Int. Math. Res. Not., (19):937-981, 2004.

[Rei74] Hans Martin Reimann. Functions of bounded mean oscillation and quasiconformal mappings. Comment. Math. Helv., 49:260-276, 1974.

[Tol03] Xavier Tolsa. Painlevé's problem and the semiadditivity of analytic capacity. Acta Math., 190(1):105-149, 2003.

[Tol05] Xavier Tolsa. Bilipschitz maps, analytic capacity, and the Cauchy integral. Ann. of Math. (2), 162(3):1243-1304, 2005. 
[UT08] Ignacio Uriarte-Tuero. Sharp examples for planar quasiconformal distortion of Hausdorff measures and removability. International Mathematics Research Notices, 2008: rnn047-43, 2008.

[Väi86] Jussi Väisälä. Bi-Lipschitz and quasisymmetric extension properties. Ann. Acad. Sci. Fenn. Ser. A I Math., 11(2):239-274, 1986.

[Vol03] Alexander Volberg. Calderón-Zygmund capacities and operators on nonhomogeneous spaces, volume 100 of CBMS Regional Conference Series in Mathematics. Published for the Conference Board of the Mathematical Sciences, Washington, DC, 2003.

Institució Catalana de Recerca i Estudis Avançats (ICREA) And Departament de Matemàtiques, Universitat Autònoma de Barcelona, 08193 Bellaterra (Barcelona), CATAlONiA

E-mail address: xtolsa@math.uab.cat

$U R L:$ http://mat.uab.es/ ${ }^{\sim}$ xtolsa

Department of Mathematics, Michigan State University, East Lansing, Mi 48824, USA

E-mail address: ignacio@math.msu.edu 